\title{
A test of the Integrated Evolutionary Speed Hypothesis in a Neotropical amphibian radiation
}

\author{
Álvaro Dugo-Cota ${ }^{1}$, Santiago Castroviejo-Fisher ${ }^{2,3}$, Carles Vilà ${ }^{1}$ \& Alejandro Gonzalez-Voyer ${ }^{1,4,5}$ \\ ${ }^{1}$ Conservation and Evolutionary Genetics Group, Estación Biológica de Doñana (EBD-CSIC), Av. Américo Vespucio s/n, 41092, Sevilla, Spain. \\ ${ }^{2}$ Laboratório de Sistemática de Vertebrados, Pontifícia Universidade Católica do Rio Grande do Sul (PUCRS), Av. Ipiranga 6681, 90619-900, Porto Alegre, \\ Brazil ${ }^{3}$ Department of Herpetology, American Museum of Natural History (AMNH), New York, USA ${ }^{4}$ Department of Zoology / Ethology, Svante \\ Arrheniusväg 18B, Stockholm University, SE-10691, Stockholm, Sweden ${ }^{5}$ Laboratorio de Conducta Animal, Instituto de Ecología, Circuito Exterior A.P. 70- \\ 275, Ciudad Universitaria, Universidad Nacional Autónoma de México, México, D.F., 04510, México. \\ *Corresponding author: A. Dugo-Cota (dugocota@ebd.csic.es)
}

\begin{abstract}
Aim The Evolutionary Speed Hypothesis is a mechanistic explanation for the latitudinal biodiversity gradient. The recently extended Integrated Evolutionary Speed Hypothesis (IESH) proposes that temperature, water availability, population size and spatial heterogeneity influence rates of molecular evolution which, in turn, affect diversification. However, the evidence for some of the associations predicted by the IESH is not conclusive and in some cases, contradictory.
\end{abstract}

Location Neotropics

Methods Using a comparative Bayesian method we tested the following predictions of the IESH: the association between rate of molecular evolution and temperature (and elevation and latitude, as proxies), water availability (using precipitation and relative humidity as proxies), productivity, and rate of diversification. We also accounted for the potential confounding effects of body size and UVB radiation. We tested these predictions separately in mitochondrial and nuclear genes.

Results Substitution rates of mitochondrial and nuclear genes were positively associated with temperature and negatively with elevation, while only the mitochondrial coding gene rate was associated with UVB radiation. However, when controlling for temperature, the association between substitution rate and elevation and UVB radiation disappeared, while a negative association with precipitation emerged. Moreover, diversification events were positively correlated with the rate of molecular evolution but only in mitochondrial genes.

Main conclusions Our results support two key predictions of the IESH. They highlight the important association between rate of molecular evolution and temperature within a recently diverged group and also confirm the positive association between molecular evolution and diversification rate, although only in mitochondrial genes. However, the lack of association between diversification and temperature and low effect size of the relationship between substitution rates and diversification in mitochondrial genes emphasize the important role other factors, such as time, spatial heterogeneity and population size might have in the origin and maintenance of the latitudinal biodiversity gradient.

Key words: Amphibians, diversification, evolutionary speed hypothesis, latitudinal biodiversity gradient, molecular evolution, substitution rate, temperature. 


\section{INTRODUCTION}

The striking difference in biodiversity between tropical and temperate regions is probably the oldest pattern described in ecology (Hawkins, 2001). Despite previous attempts at explaining the latitudinal biodiversity gradient, the underlying drivers remain elusive (Hillebrand, 2004; Jablonski et al., 2006; Mittelbach et al., 2007). There is nonetheless a certain consensus that this pattern must be the result of latitudinal differences in the rates of speciation, extinction and migration, or any combination thereof (Dowle et al., 2013). Many studies have attempted to explain the latitudinal biodiversity gradient by focusing on the factors that influence the rate of molecular evolution and its effect on the rate of diversification (reviewed in Dowle et al., 2013). Among them, the explanation having attracted most attention is the 'Evolutionary Speed Hypothesis'. Rensch (1959) suggested that organisms in warmer environments have shorter generation times, which increases the pace of selection and therefore the evolutionary speed. The idea was extended by Rohde (1992) who proposed that organisms in warmer environments have a higher rate of molecular evolution via shorter generation times and higher mutation rate that lead to faster adaptation and differentiation. Recently, Gillman \& Wright (2014) proposed an integrative version of the hypothesis recognising that the relationship between temperature and rate of molecular evolution is not monotonic, and that in hot climates limited water availability may in fact lead to reduced rates of molecular evolution either directly (e. g. Goldie et al., 2010) or indirectly through its effect on primary productivity. The latter is also proposed to influence the rate of molecular evolution based on the fact that it is a good predictor of species richness (Gillman \& Wright 2014). In addition, the effects of population size and spatial heterogeneity, which can favour isolation of populations as well as the potential for new mutations to be fixed, were also incorporated into the hypothesis. These additions to the original Evolutionary Speed Hypothesis resulted in the "Integrated Evolutionary Speed Hypothesis" (hereafter, IESH; Gillman \& Wright 2014). The predicted end result of the faster rate of molecular evolution is an increased rate of speciation, and therefore the IESH is proposed as a mechanistic explanation of the higher diversity observed in tropical latitudes (Gillman \& Wright 2014).

A diversity of taxa, including plants, invertebrates, fishes, birds, mammals, reptiles and amphibians, show a higher rate of molecular evolution in warmer environments, apparently supporting the IESH (e.g. Davies et al., 2004; Gillooly et al., 2005; Gillman et al., 2009; Wright et al., 2010; Gillman et al., 2012; Lourenço et al., 2013). Nonetheless, a limitation of these studies is that in most of them elevation or latitude were used as proxies for temperature (but see Davies et al., 2004; Gillooly et al., 2005), and while elevation and latitude can reflect differences in temperature they are also associated with other factors that may influence rate of molecular evolution (e.g. uv radiation, oxygen stress, population size, seasonality). Hence, although available evidence is consistent with the predicted positive association between temperature and rate of molecular evolution, it is not conclusive (Gillman \& Wright 2014). Finally, and perhaps most importantly, only a single study has simultaneously tested two of the key predictions of the IESH (Davies et al., 2004): the increase in rate of molecular evolution in warmer environments and the association between rate of molecular evolution and diversification. This study did not find support for the association between rate of molecular evolution and diversification, casting doubts on the generality of the IESH. Furthermore, to our knowledge, only one study to date has found the predicted positive relationship between water availability and rate of molecular evolution (Goldie et al., 2010), while the predicted positive association between the latter and productivity remains untested.

Here, using the Neotropical amphibian family Centrolenidae as our model system, we tested four of the key predictions of the IESH and also analyzed the effects of commonly used proxies and potential confounding factors. More specifically we tested the following predicted positive associations between rate of molecular evolution and: firstly temperature, secondly water availability (measured as precipitation and relative humidity), thirdly primary productivity, and finally rate of diversification. In 
addition we tested the effect of variables that are either commonly used as proxies for temperature - i.e. latitude and elevation - or could possibly have confounding effects on the predicted relationships, i.e. UVB radiation and body size. We used three proxies for the rate of molecular evolution: the synonymous and non-synonymous substitution rates (henceforth $\mathrm{dS}$ and $\mathrm{dN}$, respectively), and the ratio of non-synonymous to synonymous substitutions (dN/dS, hereafter $\omega)$. We tested all associations in both nuclear and mitochondrial genes using a whole-tree method (Lartillot \& Poujol, 2011).

Centrolenidae, commonly known as glass-frogs, because of their transparent or semi-transparent venter, is a diverse group of arboreal frogs comprised of more than 140 species included in 12 genera, originating from a relatively recent radiation (23.4 mya; 95\% highest posterior density: 19.6-28.82 mya) (Guayasamin et al., 2009; CastroviejoFisher et al., 2014). The family presents a wide Neotropical distribution, from southern Mexico to Bolivia with an isolated group in southeastern Brazil and northeastern Argentina (Fig. 1) (Guayasamin et al., 2009; CastroviejoFisher et al., 2014). Centrolenids are also ecologically diverse as they are found in very distinct habitats, from sea level to high mountain ranges (3300 m.a.s.l.) (Guayasamin et al., 2009). Furthermore, Centrolenids are highly dependent on water as females lay their eggs on leaves over-hanging streams, and when the eggs hatch, tadpoles fall into the water where they complete their development. Recently, a well-resolved molecular phylogeny of Centrolenidae combining mitochondrial and nuclear genes and with high species coverage has become available (Castroviejo-Fisher et al., 2014). Hence, Centrolenidae offers an excellent model system to analyse the influence of environmental energy on the rate of molecular evolution in a diverse but recently diverged group of species.

\section{MATERIAL AND METHODS}

\section{Taxa and Molecular Data}

The study included 97 Centrolenidae species, some of which are yet to be named and described (CastroviejoFisher et al., 2014). The sample represents $>65 \%$ of the estimated species richness of the clade and includes representatives from all recognized genera and habitats in which these species are present. We used the most recent and most complete phylogenetic reconstruction based on maximum likelihood inference combining mitochondrial and nuclear genes (Castroviejo-Fisher et al., 2014). Prior to each analysis the tree was pruned to include only species for which we had accurate phenotypic, environmental and genetic data.

We used 3 mitochondrial (total 2.8kb) and 7 single-copy nuclear gene fragments (total $3.5 \mathrm{~kb}$ ). These genes (length, nucleotide diversity $[\pi]$ (Nei \& Li, 1979) and Watterson estimator [ $\theta$ ] (Watterson, 1975) considering only one sequence per species) are the mitochondrial rRNA genes $12 \mathrm{~S}$ (975bp, $\pi=0.071, \theta=70.14$ ) and $16 \mathrm{~S}$ (892bp, $\pi=0.096$, $\theta=76.94) ;$ the mitochondrial protein-coding $\mathrm{NADH}$ dehydrogenase subunit 1 (ND1, 979bp, $\pi=0.167, \theta=94.83$ ); and the nuclear protein-coding brain-derived neurotrophic factor (BDNF, 696bp, $\pi=0.009, \theta=13.86$ ), proto-oncogene cellular myelocytomatosis (C-MYC, 423bp, $\pi=0.044$, $\theta=24.50$ ), chemokine receptor 4 (CXCR4, 354bp, $\pi=0.029$, $\theta=18.67$ ), proopiomelanocortin A (POMC, 615bp, $\pi=0.032$, $\theta=30.32$ ), recombination activating gene-1 (RAG-1, 456bp, $\pi=0.031, \theta=22.47)$ and solute-carrier family 8 members 1 and 3 (SLC8A1, 540bp, $\pi=0.024, \theta=21.42$; SLC8A3, 465bp, $\pi=0.011, \theta=13.23)$. Not all gene sequences were available for all species. A complete list of genes used per species with their GenBank accession number is available in Appendix S1 in Supporting Information.

Prior to analysing protein-coding genes, we translated them in EMBOSS Transeq (Rice et al., 2000) to identify the correct open reading frame and compared the sequences to the same gene in the most closely related species using BLASTn (Altschul et al., 1990). For analyses, sequences were concatenated as follows to minimize confounding effects due to potential different effects of traits on the mitochondrial and nuclear genomes: mitochondrial noncoding genes (12S and 16S; $1867 \mathrm{bp}, \mathrm{n}=97$ species) and nuclear protein-coding genes (BDNF, C-MYC, CXCR4, POMC, RAG-1, SLC8A1 and SLC8A3; 3549bp, $n=83$ species). The single mitochondrial protein-coding gene ND1 (960 bp, $\mathrm{n}=91$ species) was analysed separately. 


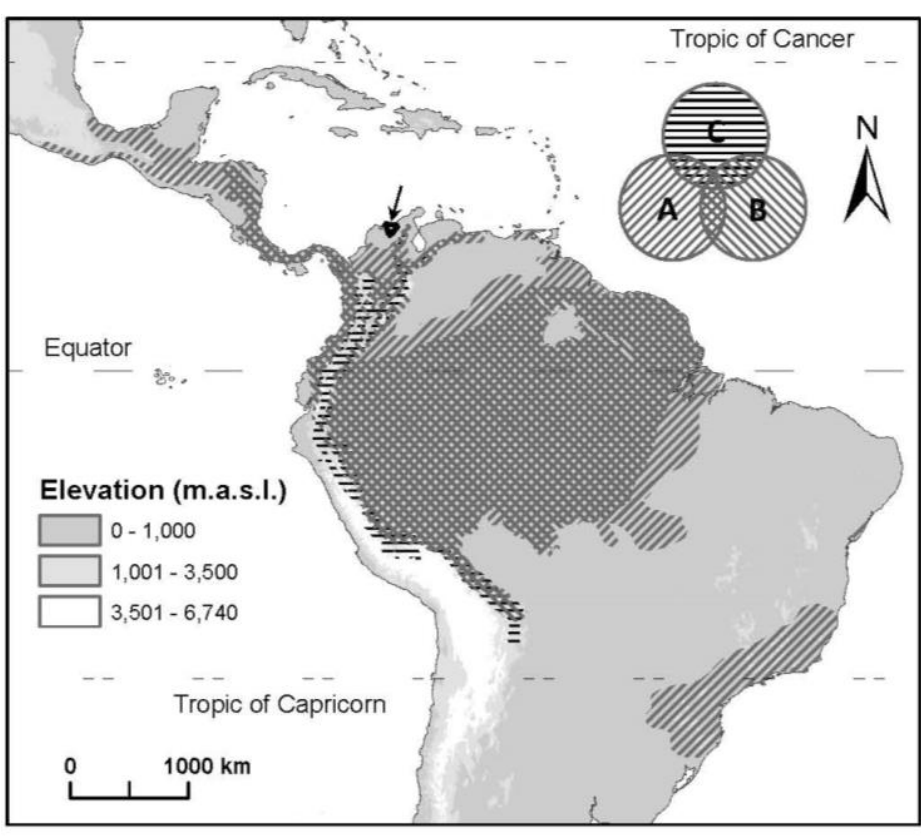

Figure 1. Geographic distribution of Centrolenidae. Clade A (right-angled lines; Teratohyla + Sachatamia + Rulyrana + Cochranella + Espadarana + Chimerella + Vitreorana) and Clade B (left-angled lines; Hyalinobatrachium + Celsiella) are basically distributed in lowlands, while Clade C (horizontal lines; Nymphargus + Centrolene) is mainly distributed in the Andes range. The arrow and the black area indicate the distribution of Ikakogi tayrona, a centrolenid species outside the three major clades, which also inhabits a mountain range. These three main clades and Ikakogi tayrona are represented on the phylogeny shown in Fig. 2, which graphically represents the association between temperature and synonymous substitution rate in the nuclear genes.

\section{Traits}

We used midpoint elevation, midpoint latitude, mean annual temperature, mean annual precipitation, mean annual relative humidity, mean net primary productivity and mean annual UVB radiation across each species' distribution to characterize correlations between environmental traits and the rate of molecular evolution. Known centrolenid distributions were adjusted based on recent sampling and corrected misidentifications (details in Appendix S2), and analysed with ArcGIS 10 (Environmental Systems Research Institute, 2011) to obtain the values of all these traits, except midpoint elevation. Midpoint elevation values were obtained from the IUCN database (2012), published literature, and our own unpublished data (details in Appendix S3). Midpoint latitude was estimated using the addXY command with the Geospatial Modelling Environment (GME) extension (Spatial Ecology, 2012). We used the absolute value of the latitude midpoint, because our interest lay in the distance to the equator regardless of the location in the northern or southern hemisphere. We obtained temperature and precipitation data (30 arcseconds resolution; $1 \mathrm{~km}$ ) from the WorldClim climatic maps (Hijmans et al., 2005), relative humidity (30 arcminute; $60 \mathrm{~km}$ ) from the Atlas of the Biosphere (New et al., 1999), net primary productivity data (15 arc-minute; $28 \mathrm{~km}$ ) from the HANNP datasets (Imhoff et al., 2004; Imhoff \& Bounoua, 2006) and UVB radiation data (15 arcminute resolution; $28 \mathrm{~km}$ ) from the glUV dataset (Beckmann et al., 2014). We obtained the mean value for each trait for each species' distribution including only distribution points within the known altitudinal range. One might wonder about the relevance of studying the influence of UVB on nocturnal animals such as centrolenids. However, Cisneros-Heredia and McDiarmid (2007) reported that absence of pigments from ventral skin (i.e. transparent parietal peritoneum) was associated with presence of pigments (i.e. iridophores) covering viscera (e.g. liver, digestive track). These authors hypothesized that pigments covering viscera protect enzymatic activity against the potentially detrimental effects of light and temperature, which could be linked to the rate of molecular evolution via mutagenesis. Finally, we obtained lineage-specific cladogenetic events (a proxy for diversification events) by counting the number of nodes between the root and each tip of the phylogeny, excluding the root node (sensu Freckleton et al., 2008) (Appendix S4). We note that although the latter is a nonparametric method, which assumes that rate of extinction is equal across all clades and time, a recent study suggests that extinction in Centrolenidae plays a minor role in explaining current richness patterns (Castroviejo-Fisher et al., 2014). All data are shown in Appendix S3.

To control for the potentially confounding effects of generation time, longevity and metabolic rate (for which no data are available) we used body size as a proxy. Body size is positively associated with generation time (Galtier et al., 2009) and longevity (Nabholz et al., 2008), and 


\begin{tabular}{|c|c|c|c|c|c|c|c|c|}
\hline & & \multicolumn{4}{|c|}{ MITOCHONDRIAL GENES } & \multirow{2}{*}{\multicolumn{3}{|c|}{$\frac{\text { NUCLEAR GENES }}{\text { Coding }}$}} \\
\hline & & \multirow{2}{*}{$\begin{array}{c}\text { Non-coding } \\
\text { Sub. rate }\end{array}$} & \multicolumn{3}{|c|}{ Coding } & & & \\
\hline & & & dS & $d N$ & $\omega$ & dS & dN & $\omega$ \\
\hline \multirow{3}{*}{$\begin{array}{l}\text { Body } \\
\text { Size }\end{array}$} & cov & -0.03 & -0.02 & 0.00 & 0.00 & -0.01 & -0.01 & -0.01 \\
\hline & $r^{2}$ & 0.04 & 0.04 & 0.00 & 0.01 & 0.02 & 0.01 & 0.00 \\
\hline & $p p$ & 0.08 & 0.06 & 0.51 & 0.62 & 0.25 & 0.30 & 0.40 \\
\hline \multirow{3}{*}{$\begin{array}{l}\text { Div. } \\
\text { events }\end{array}$} & cov & 0.06 & 0.03 & 0.03 & -0.00 & 0.01 & 0.01 & 0.01 \\
\hline & $r^{2}$ & 0.10 & 0.06 & 0.03 & 0.00 & 0.00 & 0.00 & 0.00 \\
\hline & $p p$ & 1.00 & 0.97 & 0.86 & 0.51 & 0.60 & 0.56 & 0.56 \\
\hline \multirow{3}{*}{ Elev. } & cov & $-0.31 *$ & $-0.16 *$ & $-0.20 *$ & -0.00 & $-0.21 *$ & 0.02 & 0.20 \\
\hline & $r^{2}$ & 0.14 & 0.08 & 0.10 & 0.00 & 0.24 & 0.00 & 0.12 \\
\hline & $p p$ & 0.00 & 0.03 & 0.05 & 0.52 & 0.01 & 0.55 & 0.92 \\
\hline \multirow{3}{*}{ Lat. } & cov & -0.12 & -0.11 & 0.11 & 0.02 & -0.11 & -0.09 & -0.01 \\
\hline & $r^{2}$ & 0.01 & 0.01 & 0.01 & 0.00 & 0.02 & 0.00 & 0.00 \\
\hline & $p p$ & 0.28 & 0.25 & 0.65 & 0.54 & 0.25 & 0.41 & 0.48 \\
\hline \multirow{3}{*}{ Temp. } & cov & 0.07 & 0.05 & 0.05 & -0.00 & 0.04 & -0.00 & -0.04 \\
\hline & $r^{2}$ & 0.31 & 0.34 & 0.25 & 0.00 & 0.35 & 0.00 & 0.18 \\
\hline & $p p$ & 1.00 & 1.00 & 0.99 & 0.48 & 1.00 & 0.40 & 0.04 \\
\hline \multirow{3}{*}{ RH } & cov & -0.00 & 0.00 & 0.01 & 0.00 & 0.01 & -0.00 & -0.01 \\
\hline & $r^{2}$ & 0.00 & 0.01 & 0.04 & 0.00 & 0.07 & 0.01 & 0.08 \\
\hline & $p p$ & 0.49 & 0.73 & 0.81 & 0.54 & 0.87 & 0.35 & 0.14 \\
\hline \multirow{3}{*}{ NPP } & cov & 0.06 & -0.03 & 0.04 & 0.00 & 0.06 & 0.01 & -0.05 \\
\hline & $r^{2}$ & 0.02 & 0.01 & 0.01 & 0.00 & 0.10 & 0.00 & 0.04 \\
\hline & $p p$ & 0.82 & 0.32 & 0.69 & 0.48 & 0.92 & 0.49 & 0.18 \\
\hline \multirow{3}{*}{ Precip. } & cov & $-0.01+$ & $-0.01+$ & 0.04 & 0.00 & 0.06 & -0.06 & -0.13 \\
\hline & $r^{2}$ & 0.00 & 0.00 & 0.01 & 0.00 & 0.07 & 0.04 & 0.17 \\
\hline & $p p$ & 0.41 & 0.46 & 0.70 & 0.53 & 0.86 & 0.23 & 0.06 \\
\hline \multirow{3}{*}{ UVB } & $\operatorname{cov}$ & 0.00 & $-0.02 *$ & -0.01 & -0.00 & -0.01 & 0.01 & 0.01 \\
\hline & $r^{2}$ & 0.00 & 0.07 & 0.03 & 0.00 & 0.04 & 0.00 & 0.03 \\
\hline & $p p$ & 0.53 & 0.04 & 0.22 & 0.51 & 0.19 & 0.59 & 0.76 \\
\hline
\end{tabular}

Table 1. Covariance matrix between substitution rate (Subst. rate in mitochondrial non-coding genes or synonymous (dS) and non-synonymous $(\mathrm{dN})$ substitution rates, and the ratio of non-synonymous to synonymous substitutions $(\omega)$ in mitochondrial and nuclear coding genes) and body size, diversification events (Div. events), elevation (Elev.), latitude (Lat.), temperature (Temp.), relative humidity $(\mathrm{RH})$, net primary productivity (NPP), preciptiation (Precip.) and UVB radiation (UVB). We show covariances (cov), which indicate the direction of the correlation, the effect size $\left(r^{2}\right)$ and the posterior probabilities (pp); values $\leq$ 0.05 or $\geq 0.95$ can be taken as indicating that the relationships are extremely unlikely to occur by chance and are shown in bold. When controlling for the effect of temperature: correlations indicated by * are no longer significant, while those indicated by + become significant (see details in the Results section and in Appendix S7).

negatively associated with metabolic rate (Martin \& Palumbi, 1993), all of which might influence the rate of molecular evolution (i.e. Gillooly et al., 2005; Nabholz et al., 2008; Lartillot \& Poujol, 2011; Santos, 2012). We used body size, measured as the midpoint of snout-vent length (SVL; tip of snout to anterior margin of cloaca). Midpoint SVL was obtained from the IUCN database (2012), published literature, and our own unpublished data (details in Appendix S3). Estimates of midpoint SVL included both males and females.

\section{Analyses}

An essential first step, prior to testing whether there is any association between phenotypic traits or environmental variables and rate of substitution, is to discard any potential confounding effects of the node-density artefact, an underestimation of the branch lengths in areas of the tree with few taxa (Venditti et al., 2006). We tested for the presence of the node-density artefact using the Test for Punctuational Evolution and the Node-Density Artifact available online (http://www.evolution.reading.ac.uk/pe/index.html) (Webster et al., 2003; Venditti et al., 2006). Presence of a node-density effect was discarded $(\delta<1)$.

To analyse the association between the rate of molecular evolution and the different traits we used Coevol (v1.4) (Lartillot \& Poujol, 2011). We analysed correlations between traits and $d S, d N$ and $\omega$. Note that only an overall 
substitution rate can be calculated for non-coding genes (12S and 16S mitochondrial rRNA). Coevol estimates the correlations jointly modelled as a multivariate Brownian diffusion process along all branches of the tree (whole-tree method). 'Whole-tree' methods now enable analyses including all the information obtained from a phylogenetic reconstruction of the clade of interest taking into account the phylogenetic dependence (Lanfear et al., 2010). The input was the multiple sequence alignment, a matrix of log-transformed continuous traits for the same taxa, and the phylogenetic tree. A covariance matrix is estimated using a Bayesian Markov Chain Monte Carlo (MCMC) method. We used a geodesic averaging method for computing branch-specific mean values of the molecular evolution parameters, as it is suggested to be more precise than an arithmetic averaging method (Lartillot \& Poujol, 2011). We ran each analysis twice until stabilization of all estimated parameters, which was visually verified by plotting the trace file of each trait. We ensured convergence by comparing the two independent runs of each analysis using the tracecomp module of Coevol. Burnin was set after stabilization (checked visually). To compute posterior estimates of the covariance matrix, we used the component readcoevol. We tested the key association between temperature and diversification predicted by the IESH using phylogenetic generalized least squares (Martins \& Hansen 1997) in the R package 'caper' (Orme et al. 2012) to control for phylogenetic nonindependence. Analyses were run under a Brownian motion model of evolution with the lambda parameter, which estimates the necessary correction for phylogenetic non-independence of the residuals (Freckleton et al., 2002; Revell 2010). Data were log transformed prior to analyses to meet assumptions of the model of evolution. Figure 2, depicting the main result of these analyses, was constructed using the phytools package (Revell, 2012) in R (R Development Core Team, 2012).

\section{RESULTS}

We confirmed the convergence of the two independent runs of each analysis. In all cases effective sample sizes of parameters and discrepancy between independent runs were well within range of values for the runs to be considered as good, with a single exception, a run for which some values were in the range for it to be considered as acceptable (see Appendix S5 for details). The number of cycles, burnin and number of sampled iterations in the posterior distribution for all analyses are shown in Appendix S6.

An overview of the main results is presented in Table 1 (see Appendix S7 for a comprehensive description). Following the prediction of the IESH, substitution rate and dS were positively correlated with temperature in mitochondrial non-coding genes, and mitochondrial and nuclear coding genes (Fig. 2). Temperature was only positively correlated with $\mathrm{dN}$ in the mitochondrial coding gene and negatively correlated with $\omega$ in the nuclear genes. However, contrary to the predictions of the IESH substitution rates were not correlated with mean annual precipitation, mean annual relative humidity or mean annual net primary productivity for the mitochondrial or the nuclear genes. Finally, in agreement with the IESH, the substitution rate in the mitochondrial non-coding genes as well as dS in the mitochondrial coding gene were positively correlated with diversification rate. However, $d N$ and $\omega$ in the mitochondrial coding gene and substitution rates ( $d N$ or $\mathrm{dS}$ ) and $\omega$ in the nuclear genes were not correlated with diversification rate.

With regard to potential confounding factors and commonly used proxies for temperature we found that, firstly, body size was not correlated with substitution rate ( $d S$ or $d N$ ) or $\omega$ in the mitochondrial or the nuclear genes, thus we can rule out confounding effects of body size. Secondly, midpoint latitude was not correlated with substitution rates or $\omega$ in the mitochondrial or the nuclear genes. On the other hand, midpoint elevation was negatively correlated with the rate of substitution in mitochondrial non-coding genes, and also with the rate of synonymous (dS) and non-synonymous (dN) substitution in the mitochondrial coding gene, but it was only correlated with the rate of synonymous substitution (dS) in the nuclear coding gene. Midpoint elevation was not correlated with the ratio of non-synonymous to synonymous substitutions $(\omega)$ in any gene. Finally, contrary to what we expected, UVB radiation was negatively 
correlated with $\mathrm{dS}$ in the mitochondrial coding gene while all other relationships were non-significant.

Interestingly, some of the significant correlations described above disappeared and others became significant when tested by controlling for other factors with which they were correlated. The partial correlations we analysed suggest that elevation and UVB radiation are correlated with the rate of molecular evolution via temperature, since their association with rate of molecular evolution disappeared when controlling for the effect of temperature. Surprisingly, when controlling for temperature we found a significantly negative correlation between precipitation and substitution rate and $\mathrm{dS}$ in mitochondrial non-coding genes and the mitochondrial coding gene, respectively.

\section{DISCUSSION}

Temperature is the key factor related with the rate of molecular evolution

The bivariate correlations show that the rate of molecular evolution is significantly correlated with temperature, elevation and UVB radiation (Table 1 ). The IESH predicts that these factors, with the exception of UVB radiation, are associated directly or indirectly with the rate of molecular evolution, and all were included within the idea of biologically available energy (Wright et al., 2006). According to the IESH, ectothermic species living in environments with higher biologically available energy have higher body temperature and therefore higher metabolic rate (Allen et al., 2006; Wright et al., 2010), increasing the production of reactive oxygen species that may lead to higher mutation rate. A recent study with poison frogs empirically tested the relationship between metabolic rate and rate of molecular evolution and showed that indeed higher active metabolic rate, but not resting metabolic rate, is associated with higher rate of molecular evolution in both mitochondrial and nuclear genes (Santos, 2012). We propose that the observed correlations between temperature, elevation, UVB radiation and the rate of molecular evolution are the result of an increased rate of mutation in warmer environments, in support of the IESH. Indeed, the fact that the correlations between rate of molecular evolution and elevation or UVB radiation disappear when we control for temperature supports our interpretation and emphasizes the crucial role of temperature as the principal factor behind these associations. The absence of an association between latitude and the rate of molecular evolution can be explained by the lack of correlation between latitude and temperature (Pearson's $r=0.07$ ). Centrolenid species present a mainly tropical distribution and this limited variation in the latitudinal range (Fig. 1) is a potential explanation for why variation in temperature is linked to altitudinal rather than latitudinal differences. Our results also stress the importance of testing the IESH using the environmental variable proposed to play the key role, temperature, rather than proxies as these may not always accurately reflect differences in environmental temperature.

According to IESH, reduced water availability is proposed to limit the rate of molecular evolution, as was observed in Australian plants (Goldie et al. 2010). Somewhat contrary to this prediction, we found that precipitation is negatively correlated with substitution rate and dS in mitochondrial non-coding genes and the mitochondrial coding gene, respectively, but only after controlling for the effect of temperature. It is certainly possible that, since centrolenids require a relatively wet microhabitat, our data measured over the entire distribution range does not properly reflect water stress. Relative humidity, however, was not associated with the rate of molecular evolution. We propose two non-mutually exclusive explanations for why, for a given temperature, in drier conditions centrolenids present higher rate of molecular evolution in mitochondrial genes. On the one hand, under hydric stress mitochondria might produce higher amounts of reactive oxygen species, which could in turn lead to higher mutation rate. Alternatively, it is possible that in adverse conditions the population size of centrolenids, which depend on liquid water for reproduction, presents more pronounced demographic fluctuations resulting in higher rate of molecular evolution (Balloux \& Lehmann, 2011). Our results do not allow us to distinguish between these two potential explanations. 


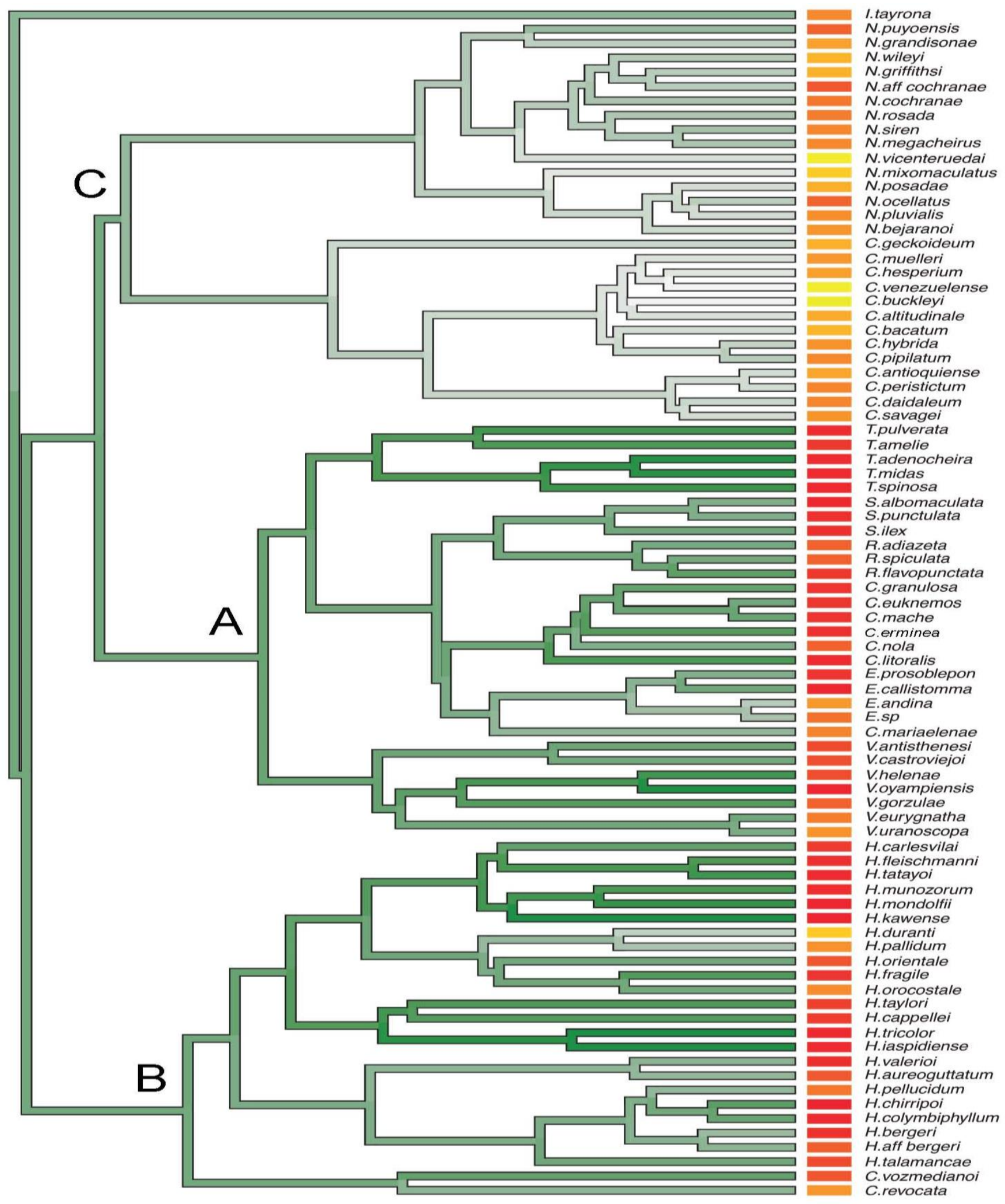

Figure 2. Phylogenetic tree of Centrolenidae. Colours of branches represent the synonymous substitution rate (dS) of the nuclear genes: where the rate of substitution increases from light grey to dark green. Rectangles at the tips of the phylogeny represent the mean annual temperature of the species' distribution range (mean annual temperature increases from yellow to red). This figure highlights the faster synonymous substitution rate in species inhabiting warmer habitats (Clades A and B versus $\mathrm{C}$ ). Letters indicate the three major clades whose distribution is shown in Fig. 1. 
Contrary to the prediction of the IESH, we did not find any correlation between net primary production and the rate of molecular evolution (Table 1) (Gillman \& Wright, 2014). We cannot rule out that there is insufficient variation in net primary productivity in this mainly tropical clade for a significant association to be detected. Nonetheless, we note that the relationship is based on the fact that productivity is a good predictor of species richness. But, as discussed below, the rate of molecular evolution may not be directly associated with species richness, as other factors may also play important roles therefore mitigating the correlation between molecular evolution and primary productivity. The absence of a correlation between productivity and rate of molecular evolution suggests that the relationship between temperature and/or water availability with the rate of molecular evolution might not be via productivity.

In sum, our results are in accord with previous studies in ectotherms (Wright et al., 2010; Lourenço et al., 2013) and endotherms (Gillman et al., 2009, 2012) that found faster substitution rates for species living in warmer environments. The observed discrepancies between mitochondrial and nuclear data might be due to differences in the statistical power to detect associations or natural differences between both kinds of genomes, such as the lower effective population size (Wright 1931) and the higher mutation rate of the mitochondrial genome (Martin and Palumbi 1993).

\section{Elevated diversification is correlated with increased rate of molecular evolution in mitochondrial genes}

The IESH also predicts that the faster rate of molecular evolution in warmer environments favours diversification because of the more rapid build-up of incompatibilities between populations (Dowle et al., 2013). Hence, a positive correlation between diversification and rate of molecular evolution would be expected. In agreement with this prediction, we found a correlation between diversification events and the rate of molecular evolution in mitochondrial genes (Table 1). However, the effect size of the correlation between substitution rates and diversification was relatively low, range from 6 to $10 \%$ (Table 1 ), indicating that other factors possibly play an important role in the association with rate of molecular evolution. The absence of a correlation between diversification events and substitution rates in nuclear genes (Table 1 ) is surprising and counter to results of theoretical and empirical studies (Ohta, 1992; Balloux \& Lehmann, 2011; Buschiazzo et al., 2012; Popadin et al., 2013). As mentioned above, it is possible that our sample of nuclear genes is not sufficient to allow us to detect the association, in contrast with previous studies (e.g. Barraclough \& Savolainen, 2001; Lanfear et al., 2010). Compared to the mitochondrial genome, the nuclear genome has a higher effective population size that can dilute the effect of demographic fluctuations associated with diversification events (Popadin et al., 2013).

\section{Body size is not correlated with the rate of molecular evolution}

We did not find any correlation between body size and substitution rates or $\omega$ in mitochondrial or nuclear genes (Table 1). This result contrasts with previous findings in mantellid and poison frogs (Wollenberg et al., 2011; Santos, 2012). The absence of a relationship between body size and the rate of molecular evolution in Centrolenidae may be a result of the reduced variation in body size in this clade. Excluding the exceptionally large Centrolene geckoideum (71.3 $\mathrm{mm} \mathrm{SVL}$ ), the largest species is only twice as large as the smallest Centrolenidae (SVL range: 18.5-36.4 mm). On the other hand, the difference between the smallest and largest species is one order of magnitude or more in mantellids (SVL range: 10-110 mm SVL) and poison-frogs (range: 0.2-6 g). The lack of correlation between body size and molecular evolution rate places the focus on the influence of environmental variables ruling out potential confounding effects of allometry.

\section{Do our results support the Integrated Evolutionary Speed Hypothesis?}

The integrated evolutionary speed hypothesis is proposed as an explanation for the latitudinal species gradient (Gillman \& Wright, 2014). Our results support two key predictions of the IESH in a recently diverged clade of Neotropical amphibians. Firstly, we found that the rate of molecular evolution is positively correlated with 
temperature. Secondly, we found that the rate of molecular evolution is positively associated with diversification, although only in mitochondrial genes and with a small effect size $\left(r^{2}=6-10 \%\right)$. Hence, at first sight our results appear to support the IESH. However, our results do not support the additional tested predictions of the IESH: namely the positive associations between rate of molecular evolution and productivity or water availability. The absence of a correlation between temperature and diversification events in centrolenids $(\lambda=1, \beta=0.16187$, s.e. $=0.1543, \quad t$-value $=1.0491, \quad p$-value $=0.2968)$ is not necessarily contrary to the IESH. As indicated by Rohde (1992) and Gillman and Wright (2014), the relationship between temperature and speciation (or species richness) is indirect and other key factors intervene, such as time or possibility of genetic isolation. A recent study points to the early colonization of mountain ranges, in combination with time allowing for richness to build up, as potential explanations for Centrolenid diversity (Hutter et al., 2013) and other studies of Neotropical amphibians also suggest mountain ranges play a key role favouring isolation of populations and therefore diversification (e.g. GonzalezVoyer et al., 2011; Santos et al., 2009). We suggest that while lowland habitats are indeed associated with higher rate of molecular evolution, as a result of higher temperature, they nonetheless lack the ecological and geographical features favouring isolation of populations, and hence speciation, compared with mountain ranges such as the Andes. A recent study supports this suggestion as it found higher genetic divergences among frog species in mountains compared to lowlands (Guarnizo \& Cannatella, 2013). Spatial heterogeneity therefore appears to overlay the important effect of temperature via the rate of molecular evolution favouring speciation.

In conclusion, we found that species inhabiting warmer environments have a higher rate of molecular evolution, which increases even more in the mitochondrial genes in drier conditions. We also found that a higher rate of molecular evolution is positively correlated with the rate of diversification in mitochondrial genes. As far as we know, this is the first study to show both an association between temperature and higher rate of molecular evolution as well as a positive correlation between the rate of molecular evolution and diversification rate. These results support the IESH, emphasizing the important association between rate of molecular evolution and temperature $\left(r^{2}=25-35 \%\right)$ even within a relatively short timescale and the relationship between the latter and the rate of diversification. However, the absence of a positive correlation between temperature and diversification, together with the small effect size of the relationship between substitution rates and diversification $\left(r^{2}=6-10 \%\right)$, highlight the influence of other factors, such as time, spatial heterogeneity or population size that must be taken into consideration (Gillman \& Wright 2014).

Aknowledgements: This work was supported by projects CGL2010-21250 and CGL2013-47547-P from the Spanish Ministry of Economy and Competitiveness and by a Young Investigator grant from the Swedish Research Council (Vetenskapsrådet). A. D-C. was funded by a FPI doctoral fellowship from the Spanish Government, A. G-V. was funded by a JAE-Doc post-doctoral fellowship from the Spanish Research Council (CSIC), co-funded by the European Social Fund, and by a young investigator grant from the Swedish Research Council (Vetenskapsrådet). Members of the Conservation and Evolutionary Genetics group at the Doñana Biological Station (EBD-CSIC) commented early drafts of the manuscript. Liam Revell provided valuable advice for the elaboration of one figure. The Laboratory of GIS and Remote Sensing (LAST-EBD) assisted with data collection use of geographical information systems.

\section{REFERENCES}

Allen, A.P., Gillooly, J.F., Savage, V.M. \& Brown, J.H. (2006) Kinetic effects of temperature on rates of genetic divergence and speciation. Proceedings of the National Academy of Sciences of the United States of America, 103, 9130-9135.

Altschul, S., Gish, W. \& Miller, W. (1990) Basic local alignment search tool. Journal of Molecular Biology, 215, 403-410.

Balloux, F. \& Lehmann, L. (2011) Substitution Rates At Neutral Genes Depend on Population Size Under Fluctuating Demography and Overlapping Generations. Evolution, 66, 605-611. 
Barraclough, T.G. \& Savolainen, V. (2001) Evolutionary rates and species diversity in flowering plants. Evolution, 55, 677-83.

Beckmann, M., Václavík, T., Manceur, A.M., Šprtová, L., von Wehrden, H., Welk, E. \& Cord, A.F. (2014) glUV: a global UV-B radiation data set for macroecological studies. Methods in Ecology and Evolution, 5, 372-383.

Buschiazzo, E., Ritland, C., Bohlmann, J. \& Ritland, K. (2012) Slow but not low: genomic comparisons reveal slower evolutionary rate and higher $\mathrm{dN} / \mathrm{dS}$ in conifers compared to angiosperms. BMC evolutionary biology, 12, 8.

Castroviejo-Fisher, S., Guayasamin, J.M., Gonzalez-Voyer, A. \& Vilà, C. (2014) Neotropical Diversification seen through glassfrogs. Journal of Biogeography, 41, 66-80.

Cisneros-Heredia, D.F. \& McDiarmid, R.W. (2007) Revision of the character of Centrolenidae (Amphibia: Anura: Athesphatanura), with comments on its taxonomy and the description of new taxa of glassfrogs. Zootaxa, 1572, $1-82$.

Davies, T.J., Savolainen, V., Chase, M.W., Moat, J. \& Barraclough, T.G. (2004) Environmental energy and evolutionary rates in flowering plants. Proceedings of the Royal Society B: Biological Sciences, 271, 2195-2200.

Dowle, E.J., Morgan-Richards, M. \& Trewick, S.A. (2013) Molecular evolution and the latitudinal biodiversity gradient. Heredity, 110, 501-510.

Environmental Systems Research Institute (2011) ArcGIS Desktop 10.

Freckleton, R.P., Harvey, P.H. \& Pagel, M. (2002) Phylogenetic analysis and comparative data: a test and review of evidence. The American naturalist, 160, 712726.

Freckleton, R.P., Phillimore, A.B. \& Pagel, M. (2008) Relating traits to diversification: a simple test. The American Naturalist, 172, 102-115.

Galtier, N., Jobson, R.W., Nabholz, B., Glémin, S. \& Blier, P.U. (2009) Mitochondrial whims: metabolic rate, longevity and the rate of molecular evolution. Biology Letters, 5, 413-416.

Gillman, L.N. \& Wright, S.D. (2014) Species richness and evolutionary speed: the influence of temperature, water and area. Journal of Biogeography, 41, 39-51.

Gillman, L.N., Keeling, D.J., Ross, H.A. \& Wright, S.D. (2009) Latitude, elevation and the tempo of molecular evolution in mammals. Proceedings of the Royal Society B: Biological Sciences, 276, 3353-3359.

Gillman, L.N., McCowan, L.S.C. \& Wright, S.D. (2012) The tempo of genetic evolution in birds: body mass and climate effects. Journal of Biogeography, 39, 1567-1572.

Gillooly, J.F., Allen, A.P., West, G.B. \& Brown, J.H. (2005) The rate of DNA evolution: effects of body size and temperature on the molecular clock. Proceedings of the National Academy of Sciences of the United States of America, 102, 140-145.

Goldie, X., Gillman, L.N., Crisp, M. \& Wright, S.D. (2010) Evolutionary speed limited by water in arid Australia. Proceedings. Biological sciences / The Royal Society, 277, 2645-53.

Gonzalez-Voyer, A., Padial, J.M., Castroviejo-Fisher, S., De la Riva, I. \& Vilà, C. (2011) Correlates of species richness in the largest Neotropical amphibian radiation. Journal of Evolutionary Biology, 24, 931-942.

Guarnizo, C.E. \& Cannatella, D.C. (2013) Genetic divergence within frog species is greater in topographically more complex regions. Journal of Zoological Systematics and Evolutionary Research, 51, 333-340.

Guayasamin, J.M., Castroviejo-Fisher, S., Trueb, L., Ayarzagüena, J., Rada, M. \& Vilà, C. (2009) Phylogenetic systematics of Glassfrogs (Amphibia: Centrolenidae) and their sister taxon Allophryne ruthveni. Zootaxa, 2100, 197.

Hawkins, B.A. (2001) Ecology's oldest pattern? Trends in Ecology \& Evolution, 16, 470.

Hijmans, R.J., Cameron, S.E., Parra, J.L., Jones, P.G. \& Jarvis, A. (2005) Very high resolution interpolated climate surfaces for global land areas. International Journal of Climatology, 25, 1965-1978.

Hillebrand, H. (2004) On the generality of the latitudinal diversity gradient. The American Naturalist, 163, 192211.

Hutter, C.R., Guayasamin, J.M. \& Wiens, J.J. (2013) Explaining Andean megadiversity: the evolutionary and ecological causes of glassfrog elevational richness patterns. Ecology letters, 16, 1135-44.

Imhoff, M.L. \& Bounoua, L. (2006) Exploring global patterns of net primary production carbon supply and demand using satellite observations and statistical data. Journal of Geophysical Research, 111, 1-8.

Imhoff, M.L., Bounoua, L., Ricketts, T., Loucks, C., Harriss, R. \& Lawrence, W.T. (2004) Global patterns in human consumption of net primary production. Nature, 429, 870-873.

IUCN (2012) The IUCN Red List of Threatened Species. Version 2012.2, Available at: http://www.iucnredlist.org (accessed on December 2013).

Jablonski, D., Roy, K. \& Valentine, J.W. (2006) Out of the tropics: evolutionary dynamics of the latitudinal diversity gradient. Science, 314, 102-106.

Lanfear, R., Welch, J.J. \& Bromham, L. (2010) Watching the clock: studying variation in rates of molecular evolution 
between species. Trends in Ecology and Evolution, 25, 495-503.

Lartillot, N. \& Poujol, R. (2011) A phylogenetic model for investigating correlated evolution of substitution rates and continuous phenotypic characters. Molecular Biology and Evolution, 28, 729-744.

Lourenço, J.M., Glémin, S., Chiari, Y. \& Galtier, N. (2013) The determinants of the molecular substitution process in turtles. Journal of Evolutionary Biology, 26, 38-50.

Martin, A.P. \& Palumbi, S.R. (1993) Body size, metabolic rate, generation time, and the molecular clock. Proceedings of the National Academy of Sciences of the United States of America, 90, 4087-4091.

Martins, E.P. \& Hansen, T.F. (1997) Phylogenies and the Comparative Method: A General Approach to Incorporating Phylogenetic Information into the Analysis of Interspecific Data. The American Naturalist, 149, 646667.

Mittelbach, G.G., Schemske, D.W., Cornell, H. V, Allen, A.P., Brown, J.M., Bush, M.B., Harrison, S.P., Hurlbert, A.H., Knowlton, N., Lessios, H. A., McCain, C.M., McCune, A.R., McDade, L. A., McPeek, M. A., Near, T.J., Price, T.D., Ricklefs, R.E., Roy, K., Sax, D.F., Schluter, D., Sobel, J.M. \& Turelli, M. (2007) Evolution and the latitudinal diversity gradient: speciation, extinction and biogeography. Ecology Letters, 10, 315-31.

Nabholz, B., Glémin, S. \& Galtier, N. (2008) Strong variations of mitochondrial mutation rate across mammals--the longevity hypothesis. Molecular Biology and Evolution, 25, 120-130.

Nei, M. \& Li, W.-H. (1979) Mathematical model for studying genetic variation in terms of restriction endonucleases. Proceedings of the National Academy of Sciences of the United States of America, 76, 5269-5273.

New, M., Hulme, M. \& Jones, P. (1999) Representing 20th century space-time climate variability. I: Development of a 1961-1990 mean monthly terrestrial climatology. Journal of Climate, 12, 829-856.

Ohta, T. (1992) The nearly neutral theory of molecular evolution. Annual Review of Ecology and Systematics, 23, 263-286.

Orme, D., Freckleton, R., Thomas, G., Petzoldt, T., Fritz, S., Isaac, N. \& Pearse, W. (2012) Comparative Analyses of Phylogenetics and Evolution in R.

Popadin, K.Y., Nikolaev, S.I., Junier, T., Baranova, M. \& Antonarakis, S.E. (2013) Purifying Selection in Mammalian Mitochondrial Protein-Coding Genes Is Highly Effective and Congruent with Evolution of Nuclear Genes. Molecular Biology and Evolution, 30, 347-355.
R Development Core Team (2012) R: A language and environment for statistical computing. Vienna, Austria, ISBN 3-900051-07-0.

Rensch, B. (1959) Evolution above the species level, Methuen, London.

Revell, L.J. (2010) Phylogenetic signal and linear regression on species data. Methods in Ecology and Evolution, 1, 319-329.

Revell, L.J. (2012) phytools: an R package for phylogenetic comparative biology (and other things). Methods in Ecology and Evolution, 3, 217-223.

Rice, P., Longden, I. \& Bleasby, A. (2000) EMBOSS: the European molecular biology open software suite. Trends in Genetics, 16, 276-277.

Rohde, K. (1992) Latitudinal gradients in species diversity: the search for the primary cause. Oikos, 65, 514-527.

Santos, J.C. (2012) Fast Molecular Evolution Associated with High Active Metabolic Rates in Poison Frogs. Molecular Biology and Evolution, 29, 2001-2018.

Santos, J.C., Coloma, L. A., Summers, K., Caldwell, J.P., Ree, R. \& Cannatella, D.C. (2009) Amazonian amphibian diversity is primarily derived from late Miocene Andean lineages. PLoS Biology, 7, e56.

Spatial Ecology (2012) GME (geospatial modelling environment). Version 0.7.2. Available at: http://www.spatialecology.com/gme/.

Venditti, C., Meade, A. \& Pagel, M. (2006) Detecting the node-density artifact in phylogeny reconstruction. Systematic biology, 55, 637-643.

Watterson, G.A. (1975) On the number of segregating sites in genetical models without recombination. Theoretical Population Biology, 7, 256-276.

Webster, A.J., Payne, R.J.H. \& Pagel, M. (2003) Molecular phylogenies link rates of evolution and speciation. Science, 301, 478.

Wollenberg, K.C., Vieites, D.R., Glaw, F. \& Vences, M. (2011) Speciation in little: the role of range and body size in the diversification of Malagasy mantellid frogs. BMC Evolutionary Biology, 11, 217.

Wright, S. (1931) Evolution in Mendelian Populations. Genetics, 16, 97-159.

Wright, S.D., Gillman, L.N., Ross, H. A. \& Keeling, D.J. (2010) Energy and the tempo of evolution in amphibians. Global Ecology and Biogeography, 19, 733-740.

Wright, S.D., Keeling, J. \& Gillman, L.N. (2006) The road from Santa Rosalia: a faster tempo of evolution in tropical climates. Proceedings of the National Academy of Sciences of the United States of America, 103, 77187722. 


\section{Supporting Information}

Appendix S1 GenBank accession numbers for each species and gene.

Appendix S2 References used to adjust IUCN distributions, when necessary.

Appendix S3 Matrix of characters used in the analyses.

Appendix S4 Phylogenetic tree with the number of nodes.

Appendix S5 Convergence diagnostics.

Appendix S6 Details for the Coevol analyses.

Appendix S7 Comprehensive results of the Coevol analyses. 
Appendix S1. GenBank accession numbers for all genes used in analyses. Ribosomal DNA 12S (12S) and 16S (16S), NADH dehydrogenase subunit 1 (ND1), brain-derived neurotrophic factor (BDNF), nuclear proto-oncogene cellular myelocytomatosis (C-MYC), chemokine receptor 4 (CXCR4), proopiomelanocortin A (POMC), recombination activating gene-1 (RAG-1), solute-carrier family 8 members 1 and 3 (SLC8A1; SLC8A3).

\begin{tabular}{|c|c|c|c|c|c|c|c|c|c|c|}
\hline Species & $12 S$ & $16 S$ & ND1 & BDNF & c-MYC & CXCR4 & POMC & RAG-1 & SLC8A1 & SLC8A3 \\
\hline Celsiella revocata & EU663379 & EU663019 & EU663113 & KF534278 & EU663281 & KF534374 & EU663204 & EU663479 & KF534113 & KF534195 \\
\hline Celsiella vozmedianoi & EU663385 & EU663025 & EU663163 & - & EU663324 & KF534375 & EU663247 & EU663531 & KF534114 & - \\
\hline Centrolene altitudinale & EU663333 & EU662974 & EU663070 & KF534280 & EU663249 & KF534377 & EU663165 & EU663433 & KF534116 & KF534197 \\
\hline Centrolene antioquiense & EU663336 & EU662977 & EU663073 & KF534281 & EU663251 & KF534378 & EU663167 & EU663436 & KF534117 & KF534198 \\
\hline Centrolene bacatum & EU663337 & EU662978 & EU663074 & - & EU663252 & KF534379 & EU663168 & EU663437 & KF534118 & KF534199 \\
\hline Centrolene ballux & KF639754 & JX126954 & HG764783 & - & - & - & - & - & - & - \\
\hline Centrolene buckleyi & EU663338 & EU662979 & EU663075 & KF534282 & EU663253 & KF534380 & EU663169 & - & - & KF534200 \\
\hline Centrolene charapita & KF639760 & KF534358 & - & - & - & - & - & - & - & - \\
\hline Centrolene condor & KF639755 & JX126955 & JX187513 & - & - & - & - & - & - & - \\
\hline Centrolene daidaleum & EU663366 & EU663007 & EU663101 & KF534283 & EU663465 & KF534381 & EU663272 & EU663465 & KF534119 & KF534201 \\
\hline Centrolene geckoideum & EU663341 & EU662982 & EU663077 & - & - & KF534382 & - & EU663440 & KF534120 & KF534202 \\
\hline Centrolene heloderma & KF639757 & JX126956 & $J \times 187509$ & - & - & - & - & - & - & - \\
\hline Centrolene hesperium & EU663345 & EU662986 & EU663081 & KF534284 & EU663258 & KF534383 & KF639777 & EU663444 & KF534121 & KF534203 \\
\hline Centrolene hybrida & EU663346 & EU662987 & EU663082 & KF534285 & EU663259 & KF534384 & EU663175 & EU663445 & KF534122 & KF534204 \\
\hline Centrolene lynchi & KF639758 & JX126957 & JX187508 & - & - & - & - & - & - & - \\
\hline Centrolene muelleri & KF639759 & JX126958 & HG764785 & KF534286 & KF534458 & KF534385 & KF639778 & - & KF534123 & KF534205 \\
\hline Centrolene peristictum & EU663352 & EU662993 & EU663088 & KF534288 & EU663266 & KF534387 & EU663181 & EU663451 & KF534124 & KF534207 \\
\hline Centrolene pipilatum & EU663353 & EU662994 & EU663089 & KF534289 & KF534459 & KF534388 & KF639779 & EU663452 & KF534125 & KF534208 \\
\hline Centrolene savagei & EU663380 & EU663020 & EU663114 & KF534290 & EU663282 & KF534389 & EU663205 & EU663480 & KF534126 & KF534209 \\
\hline Centrolene venezuelense & EU663360 & EU663001 & EU663095 & KF534291 & EU663267 & KF534390 & EU663186 & EU663459 & KF534127 & KF534210 \\
\hline Chimerella corleone & KF639761 & KF534359 & - & - & - & - & - & - & - & - \\
\hline Chimerella mariaelenae & EU663350 & EU662991 & EU663086 & KF534292 & EU663263 & KF534391 & EU663179 & EU663449 & KF534128 & KF534211 \\
\hline Cochranella erminea & KF639762 & KF534360 & HG764786 & KF534293 & KF534460 & KF534392 & KF639780 & - & KF534129 & KF534212 \\
\hline Cochranella euknemos & EU663367 & EU663008 & EU663102 & - & KF534461 & KF534393 & EU663193 & EU663466 & - & KF534213 \\
\hline
\end{tabular}




\begin{tabular}{|c|c|c|c|c|c|c|c|c|c|c|}
\hline Species & $12 S$ & $16 S$ & ND1 & BDNF & c-MYC & CXCR4 & POMC & RAG-1 & SLC8A1 & SLC8A3 \\
\hline Cochranella granulosa & EU663370 & EU663010 & EU663195 & KF534294 & EU663274 & KF534394 & KF639781 & EU663469 & KF534130 & KF534214 \\
\hline Cochranella guayasamini & KF639764 & KF534362 & - & - & - & - & - & - & - & - \\
\hline Cochranella litoralis & EU663349 & EU662990 & EU663085 & KF534295 & EU663262 & KF534395 & EU663178 & EU663448 & KF534131 & KF534215 \\
\hline Cochranella mache & EU663373 & EU663013 & EU663107 & KF534296 & EU663277 & KF534396 & EU663198 & EU663472 & KF534132 & KF534216 \\
\hline Cochranella nola & EU663381 & EU663021 & EU663115 & KF534297 & EU663283 & KF534397 & EU663206 & EU663481 & KF534133 & KF534217 \\
\hline Cochranella resplendens & KF639763 & KF534361 & HG764787 & - & - & - & - & - & - & - \\
\hline Espadarana andina & EU663335 & EU662976 & EU663072 & KF534298 & EU663250 & KF534398 & EU663166 & EU663435 & KF534134 & KF534218 \\
\hline "Espadarana audax" & KF639753 & KF534355 & HG764782 & - & - & - & - & - & - & - \\
\hline Espadarana callistomma & EU663340 & EU662981 & EU663076 & KF534299 & EU663255 & KF534399 & EU663171 & EU663439 & KF534135 & KF534219 \\
\hline "Espadarana durrellorum" & KF639756 & KF534356 & HG764784 & - & - & - & - & - & - & - \\
\hline Espadarana prosoblepon & EU663354 & EU662995 & AY819466 & - & AY819170 & KF534400 & AY819085 & EU663453 & KF534136 & KF534220 \\
\hline Espadarana sp & EU663355 & EU662996 & EU663090 & KF534300 & KF534462 & KF534401 & EU663182 & EU663454 & KF534137 & KF534221 \\
\hline Hyalinobatrachium aff bergeri & EU663393 & EU663026 & EU663119 & KF534301 & EU663290 & KF534402 & EU663210 & EU663485 & KF534138 & KF534222 \\
\hline Hyalinobatrachium aureoguttatum & EU663391 & EU663032 & EU663124 & KF534302 & EU663288 & KF534403 & EU663214 & EU663491 & KF534139 & KF534223 \\
\hline Hyalinobatrachium bergeri & EU663392 & EU663033 & EU663125 & KF534303 & EU663289 & KF534404 & EU663215 & EU6634921 & KF534140 & KF534224 \\
\hline Hyalinobatrachium cappellei & EU663401 & EU663040 & EU663132 & KF534304 & EU663297 & - & EU663222 & EU663499 & KF534141 & KF534225 \\
\hline Hyalinobatrachium carlesvilai & EU663388 & EU663030 & EU663122 & KF534305 & EU663291 & KF534405 & EU663212 & EU663489i & KF534142 & KF534226 \\
\hline Hyalinobatrachium chirripoi & EU663398 & EU663037 & EU663129 & KF534307 & EU663294 & KF534407 & EU663219 & EU663496 & KF534144 & KF534228 \\
\hline Hyalinobatrachium colymbiphyllum & EU663400 & EU663039 & EU663131 & KF534308 & EU663296 & KF534408 & EU663221 & EU663498 & KF534145 & KF534229 \\
\hline Hyalinobatrachium duranti & EU663402 & EU663041 & EU663133 & KF534309 & EU663298 & KF534409 & EU663223 & EU663500 & KF534146 & KF534230 \\
\hline Hyalinobatrachium fleischmanni & EU663406 & EU663045 & EU663137 & KF534310 & EU663300 & KF534410 & EU663225 & EU663504 & KF534147 & KF534231 \\
\hline Hyalinobatrachium fragile & EU663407 & EU447286 & EU663138 & KF534311 & EU663301 & KF534411 & EU663226 & EU663505 & KF534148 & KF534232 \\
\hline Hyalinobatrachium iaspidiense & EU663408 & EU663047 & EU663139 & KF534312 & EU663302 & KF534412 & - & EU663506 & KF534149 & KF534233 \\
\hline Hyalinobatrachium kawense & EU663387 & EU663029 & EU663121 & KF534314 & EU663329 & KF534414 & EU663211 & EU663488 & KF534151 & KF534235 \\
\hline Hyalinobatrachium mondolfii & EU663411 & EU663050 & EU663142 & KF534315 & EU663305 & KF534415 & EU663229 & EU663509 & KF534152 & KF534236 \\
\hline Hyalinobatrachium munozorum & EU663395 & EU663034 & EU663126 & - & KF534464 & KF534416 & EU663216 & EU663493 & - & KF534237 \\
\hline Hyalinobatrachium orientale & EU663413 & EU447289 & EU663144 & KF534316 & EU663306 & KF534417 & EU663230 & EU663511 & KF534153 & KF534238 \\
\hline Hyalinobatrachium orocostale & EU663414 & EU447284 & EU663145 & KF534317 & EU663307 & KF534418 & EU663231 & EU663512 & KF534154 & KF534239 \\
\hline
\end{tabular}




\begin{tabular}{|c|c|c|c|c|c|c|c|c|c|c|}
\hline Species & $12 S$ & $16 S$ & ND1 & BDNF & c-MYC & CXCR4 & POMC & RAG-1 & SLC8A1 & SLC8A3 \\
\hline Hyalinobatrachium pallidum & EU663415 & EU663052 & EU663146 & KF534318 & EU663292 & KF534419 & EU663217 & EU663513 & KF534155 & KF534240 \\
\hline Hyalinobatrachium pellucidum & EU663397 & EU663036 & EU663128 & KF534319 & EU663293 & KF534420 & EU663218 & EU663495 & KF534156 & KF534241 \\
\hline Hyalinobatrachium talamancae & EU663418 & EU663054 & EU663149 & KF534321 & EU663313 & KF534422 & EU663233 & EU663516 & KF534158 & KF534243 \\
\hline Hyalinobatrachium tatayoi & EU663419 & EU663055 & EU663150 & KF534322 & EU663310 & KF534423 & EU663234 & EU663517 & KF534159 & KF534244 \\
\hline Hyalinobatrachium taylori & EU663420 & EU663056 & EU663151 & KF534323 & EU663311 & KF534424 & EU663235 & EU663518 & KF534160 & KF534245 \\
\hline Hyalinobatrachium tricolor & EU663386 & EU663027 & HG764789 & KF534324 & EU663328 & KF534425 & - & EU663486 & KF534161 & KF534246 \\
\hline Hyalinobatrachium valerioi & EU663421 & EU663058 & EU663152 & KF534325 & EU663312 & KF534426 & EU663236 & EU663519 & KF534162 & KF534247 \\
\hline Ikakogi tayrona & EU663356 & EU662997 & EU663091 & KF534326 & EU663330 & KF534427 & EU663183 & EU663455 & KF534163 & KF534248 \\
\hline Nymphargus anomalus & KF639766 & KF534364 & HG764790 & - & - & - & - & - & - & - \\
\hline Nymphargus bejaranoi & EU663422 & EU663059 & EU663155 & KF534328 & EU663314 & KF534429 & EU663239 & EU663522 & KF534165 & KF534250 \\
\hline Nymphargus chancas & KF639767 & KF534365 & HG764791 & - & - & - & - & - & - & - \\
\hline Nymphargus cochranae & EU663425 & EU663061 & EU663156 & KF534329 & EU663317 & KF534430 & EU663240 & EU663523 & KF534166 & KF534251 \\
\hline Nymphargus garciae & AY326022 & AY326022 & - & - & - & - & - & - & - & - \\
\hline Nymphargus grandisonae & EU663344 & EU662985 & EU663080 & KF534330 & EU663257 & KF534431 & EU663174 & EU663443 & KF534167 & KF534252 \\
\hline Nymphargus griffithsi & EU663426 & EU663062 & EU663157 & KF534331 & EU663318 & KF534432 & EU663241 & EU663524 & KF534168 & KF534253 \\
\hline Nymphargus megacheirus & EU663427 & EU663063 & EU663158 & - & EU663319 & KF534433 & EU663242 & EU663525 & KF534169 & KF534254 \\
\hline Nymphargus mixomaculatus & KF639768 & EU663064 & EU663159 & KF534332 & EU663320 & KF534434 & EU663243 & EU663526 & KF534170 & KF534255 \\
\hline Nymphargus ocellatus & KF639769 & KF534366 & HG764792 & KF534333 & KF534465 & KF534435 & KF639784 & KF639787 & KF534171 & KF534256 \\
\hline Nymphargus pluvialis & EU663428 & EU663065 & EU663160 & KF534334 & EU663321 & KF534436 & EU663244 & EU663527 & KF534172 & KF534257 \\
\hline Nymphargus posadae & KF639770 & KF 534367 & - & - & - & KF534437 & - & EU663528 & KF534173 & - \\
\hline Nymphargus puyoensis & KF639771 & KF534368 & HG764793 & - & - & - & - & EU663478 & - & - \\
\hline Nymphargus rosada & EU663429 & EU663066 & EU663161 & KF534335 & EU663322 & KF534438 & EU663245 & EU663529 & KF534174 & KF534258 \\
\hline Nymphargus siren & EU663430 & EU663067 & EU663162 & KF534336 & EU663323 & KF534439 & EU663246 & EU663530 & KF534175 & KF534259 \\
\hline Nymphargus vicenteruedai & EU663424 & EU663058 & EU663154 & KF534337 & EU663316 & KF534440 & EU663238 & EU663521l & KF534176 & KF534260 \\
\hline Nymphargus wileyi & EU663431 & EU663068 & EU663164 & KF534338 & EU663325 & KF534441 & EU663248 & EU663532 & KF534177 & KF534261 \\
\hline Nymphargus aff cochranae & EU663423 & EU663060 & EU663153 & KF534327 & EU663315 & KF534428 & EU663237 & EU663520 & KF534164 & KF534249 \\
\hline Rulyrana adiazeta & EU663361 & EU663002 & EU663096 & KF534339 & EU663268 & KF534442 & EU663187 & EU663460 & KF534178 & KF534262 \\
\hline Rulyrana flavopunctata & EU663368 & EU663009 & EU663103 & KF534340 & EU663273 & KF534443 & EU663194 & EU663467 & KF534179 & KF534263 \\
\hline
\end{tabular}




\begin{tabular}{|c|c|c|c|c|c|c|c|c|c|c|}
\hline Species & $12 S$ & $16 S$ & ND1 & BDNF & c-MYC & CXCR4 & POMC & RAG-1 & SLC8A1 & $\overline{S L C 8 A 3}$ \\
\hline Rulyrana spiculata & EU663382 & EU663022 & EU663116 & KF534341 & EU663284 & KF534444 & EU663207 & EU663482 & KF534180 & KF534264 \\
\hline Sachatamia albomaculata & EU663362 & EU663003 & EU663097 & KF534343 & EU663270 & KF534446 & EU663188 & EU663461 & KF534182 & KF534266 \\
\hline Sachatamia ilex & EU663347 & EU662988 & EU663083 & KF534344 & EU663260 & KF534447 & EU663176 & EU663446 & KF534183 & KF534267 \\
\hline Sachatamia orejuela & KF639773 & KF534371 & HG764794 & - & - & - & - & - & - & - \\
\hline Sachatamia punctulata & EU663378 & EU663018 & EU663112 & KF534345 & EU663280 & KF534448 & EU663203 & EU663477 & KF534184 & KF534268 \\
\hline Teratohyla adenocheira & KF639774 & KF534372 & HG764795 & KF534346 & KF534466 & KF534449 & KF639785 & КF639788 & KF534185 & KF534269 \\
\hline Teratohyla amelie & EU663365 & EU663005 & EU663099 & KF534347 & EU663327 & KF534450 & EU663190 & EU663463 & KF534186 & KF534270 \\
\hline Teratohyla midas & EU663374 & EU663014 & EU663108 & - & EU663278 & - & EU663199 & EU6634731 & - & - \\
\hline Teratohyla pulverata & EU663416 & EU663053 & EU663147 & KF534348 & EU663308 & KF534451 & EU663232 & EU6635145 & KF534187 & KF534271 \\
\hline Teratohyla spinosa & EU663383 & EU663023 & EU663117 & KF534349 & EU663285 & KF534452 & EU663208 & EU663483 & KF534188 & KF534272 \\
\hline Vitreorana antisthenesi & EU663390 & EU663031 & EU663123 & KF534350 & EU663287 & KF534453 & EU663213 & EU663490 & KF534189 & KF534273 \\
\hline Vitreorana castroviejoi & EU663363 & EU663004 & EU663098 & KF534351 & EU663271 & KF534454 & EU663189 & EU663462 & KF534190 & KF534274 \\
\hline Vitreorana eurygnatha & AY843595 & AY843595 & EU663135 & - & - & - & - & AY844383 & - & - \\
\hline Vitreorana gorzulae & EU663343 & EU662984 & EU663079 & KF534352 & EU663256 & KF534455 & EU663173 & EU663442 & KF534191 & KF534275 \\
\hline Vitreorana helenae & EU663372 & EU663012 & EU663106 & KF534353 & EU663276 & KF534456 & EU663197 & EU663471 & KF534192 & KF534276 \\
\hline Vitreorana oyampiensis & EU663377 & EU663017 & EU663111 & KF534354 & EU663326 & KF534457 & EU663202 & EU663476 & KF534193 & KF534277 \\
\hline Vitreorana uranoscopa & KF639776 & KF639775 & - & - & - & - & JX298142 & - & - & - \\
\hline
\end{tabular}


Appendix S2. References used to adjust IUCN distributions, when necessary. A dash indicates no adjustment was done.

\begin{tabular}{|c|c|}
\hline Species & IUCN Distribution adjusted according to \\
\hline Celsiella revocata & - \\
\hline Celsiella vozmedianoi & - \\
\hline Centrolene altitudinale & [1] \\
\hline Centrolene antioquiense & [2] \\
\hline Centrolene bacatum & - \\
\hline Centrolene ballux & - \\
\hline Centrolene buckleyi & [unpub. data] \\
\hline Centrolene charapita & New creation [unpub. data] \\
\hline Centrolene condor & [3] \\
\hline Centrolene daidaleum & {$[4,5]$} \\
\hline Centrolene geckoideum & [6] \\
\hline Centrolene heloderma & - \\
\hline Centrolene hesperium & - \\
\hline Centrolene hybrida & - \\
\hline Centrolene lynchi & - \\
\hline Centrolene muelleri & - \\
\hline Centrolene peristictum & - \\
\hline Centrolene pipilatum & - \\
\hline Centrolene savagei & - \\
\hline Centrolene venezuelense & {$[1$, unpub. data] } \\
\hline Chimerella corleone & New creation [unpub. data] \\
\hline Chimerella mariaelenae & - \\
\hline Cochranella erminea & {$[7]$} \\
\hline Cochranella euknemos & - \\
\hline Cochranella granulosa & - \\
\hline Cochranella guayasamini & - \\
\hline Cochranella litoralis & - \\
\hline Cochranella mache & - \\
\hline Cochranella nola & [unpub. data] \\
\hline Cochranella resplendens & - \\
\hline Espadarana andina & [1] \\
\hline "Espadarana audax" & - \\
\hline Espadarana callistomma & [8] \\
\hline "Espadarana durrellorum" & - \\
\hline Espadarana prosoblepon & [9] \\
\hline Espadarana sp & New creation [unpub. data] \\
\hline Hyalinobatrachium aff bergeri & New creation [unpub. data] \\
\hline Hyalinobatrachium aureoguttatum & - \\
\hline Hyalinobatrachium bergeri & [unpub. data] \\
\hline Hyalinobatrachium cappellei & {$[10-13]$} \\
\hline Hyalinobatrachium carlesvilai & {$[14]$} \\
\hline Hyalinobatrachium chirripoi & - \\
\hline
\end{tabular}




\begin{tabular}{|c|c|}
\hline Species & IUCN Distribution adjusted according to \\
\hline Hyalinobatrachium colymbiphyllum & - \\
\hline Hyalinobatrachium duranti & - \\
\hline Hyalinobatrachium fleischmanni & {$[15$, unpub. data] } \\
\hline Hyalinobatrachium fragile & - \\
\hline Hyalinobatrachium iaspidiense & {$[13,16,17]$} \\
\hline Hyalinobatrachium kawense & [13] \\
\hline Hyalinobatrachium mondolfii & {$[13,18]$} \\
\hline Hyalinobatrachium munozorum & {$[18$, unpub. data] } \\
\hline Hyalinobatrachium orientale & [unpub. data] \\
\hline Hyalinobatrachium orocostale & [19] \\
\hline Hyalinobatrachium pallidum & [1] \\
\hline Hyalinobatrachium pellucidum & {$[20]$} \\
\hline Hyalinobatrachium talamancae & - \\
\hline Hyalinobatrachium tatayoi & [1] \\
\hline Hyalinobatrachium taylori & [13] \\
\hline Hyalinobatrachium tricolor & [13] \\
\hline Hyalinobatrachium valerioi & - \\
\hline Ikakogi tayrona & - \\
\hline Nymphargus aff cochranae & New creation [unpub. data] \\
\hline Nymphargus anomalus & - \\
\hline Nymphargus bejaranoi & - \\
\hline Nymphargus chancas & - \\
\hline Nymphargus cochranae & [21] \\
\hline Nymphargus garciae & - \\
\hline Nymphargus grandisonae & - \\
\hline Nymphargus griffithsi & - \\
\hline Nymphargus megacheirus & - \\
\hline Nymphargus mixomaculatus & [22] \\
\hline Nymphargus ocellatus & - \\
\hline Nymphargus pluvialis & - \\
\hline Nymphargus posadae & - \\
\hline Nymphargus puyoensis & - \\
\hline Nymphargus rosada & - \\
\hline Nymphargus siren & [unpub. data] \\
\hline Nymphargus vicenteruedai & - \\
\hline Nymphargus wileyi & - \\
\hline Rulyrana adiazeta & - \\
\hline Rulyrana flavopunctata & [23] \\
\hline Rulyrana spiculata & - \\
\hline Sachatamia albomaculata & {$[24]$} \\
\hline Sachatamia ilex & - \\
\hline Sachatamia orejuela & {$[25]$} \\
\hline Sachatamia punctulata & - \\
\hline Teratohyla adenocheira & {$[26,27]$} \\
\hline
\end{tabular}




\begin{tabular}{cc}
\hline \hline Species & IUCN Distribution adjusted according to \\
\hline Teratohyla amelie & {$[$ unpub. data] } \\
Teratohyla midas & {$[21,28]$} \\
Teratohyla pulverata & - \\
Teratohyla spinosa & - \\
Vitreorana antisthenesi & - \\
Vitreorana castroviejoi & - \\
Vitreorana eurygnatha & - \\
Vitreorana gorzulae & {$[29]$} \\
Vitreorana helenae & {$[28]$} \\
Vitreorana oyampiensis & {$[29]$} \\
Vitreorana uranoscopa & {$[30-33]$} \\
\hline \hline
\end{tabular}


Appendix S3. Matrix of characters used in the analyses. References for midpoint snout Vent Length (SVL) and midpoint elevation are included. The number of diversification events (Div. events) was extracted from the maximum-likelihood phylogenetic tree (see Appendix S4). The mean annual temperature, midpoint latitude, mean annual relative humidity (RH), mean annual net primary productivity (NPP), mean annual precipitation and mean annual UVB radiation (UVB) were obtained from the species distribution using GIS after adjustment, when necessary (see details in Material and Methods and Appendix S2).

\begin{tabular}{|c|c|c|c|c|c|c|c|c|c|}
\hline Species & $\begin{array}{c}\text { Div. } \\
\text { events } \\
\text { (number) }\end{array}$ & $\begin{array}{l}\text { Midpoint Snout Vent } \\
\text { Length }(\mathrm{mm})\end{array}$ & $\begin{array}{c}\text { Midpoint } \\
\text { elevation }(\mathrm{m})\end{array}$ & $\begin{array}{c}\text { Mean annual } \\
\text { temperature } \\
\left({ }^{\circ} \mathrm{C}\right)\end{array}$ & $\begin{array}{l}\text { Midpoint } \\
\text { latitude } \\
\text { (degrees) }\end{array}$ & $\begin{array}{l}\text { Mean } \\
\text { annual } \\
\text { RH (\%) }\end{array}$ & $\begin{array}{l}\text { Mean annual } \\
\operatorname{NPP}\left(g C * 10^{9}\right)\end{array}$ & $\begin{array}{l}\text { Mean annual } \\
\text { precipitation } \\
(\mathrm{mm})\end{array}$ & $\begin{array}{c}\text { Mean } \\
\text { annual UVB } \\
\left(\mathrm{J} / \mathrm{m}^{2}\right)\end{array}$ \\
\hline Celsiella revocata & 3 & $23.5[34,35]$ & $1500[36]$ & 18.3 & 10.4 & 78.5 & 59.9 & 1228.8 & 5166.0 \\
\hline Celsiella vozmedianoi & 3 & $27.3[37,38]$ & $775[36]$ & 22 & 10.7 & 81.0 & 92.4 & 1808.0 & 5609.0 \\
\hline Centrolene altitudinale & 10 & $22.2[35,39,40]$ & $2020[36]$ & 17.1 & 7.4 & 80.5 & 43.1 & 1738.4 & 6095.3 \\
\hline Centrolene antioquiense & 7 & $20.9[41,42]$ & $2090[2,36]$ & 17.4 & 5.8 & 79.9 & 81.6 & 2948.2 & 5802.2 \\
\hline Centrolene bacatum & 8 & $20.6[38,39,45,46]$ & $2150[36]$ & 16.4 & 1.2 & 80.5 & 9.9 & 1814.1 & 6037.0 \\
\hline Centrolene ballux & 11 & $21.3[47]$ & $\begin{array}{l}1962.5 \\
{[36,48]}\end{array}$ & 16.9 & 0.4 & 75.8 & 33.3 & 1287.9 & 7036.1 \\
\hline Centrolene buckleyi & 11 & $29.9[38,49]$ & $2700[36]$ & 12.8 & 1.1 & 67.7 & 102.3 & 1925.0 & 4914.0 \\
\hline Centrolene charapita & 5 & 35.9 [unpub. data] & $\begin{array}{l}664 \text { [unpub. } \\
\text { data] }\end{array}$ & 24.3 & 5.3 & 79.8 & 50.7 & 1922.3 & 4922.7 \\
\hline Centrolene condor & 6 & $25.9[3,50]$ & $2003.5[3,36]$ & 18 & 3.6 & 77.0 & 80.1 & 2588.9 & 5027.9 \\
\hline Centrolene daidaleum & 7 & $22.4[51]$ & $1430[4,36]$ & 19.2 & 6.2 & 79.2 & 50.6 & 2094.3 & 6345.6 \\
\hline Centrolene geckoideum & 5 & $71.3[53]$ & $2137.5[36]$ & 16.8 & 3.1 & 79.0 & 43.2 & 1943.3 & 5625.2 \\
\hline Centrolene heloderma & 8 & $29.6[54]$ & $2125[36]$ & 16.7 & 2.4 & 80.3 & 29.9 & 2215.7 & 5540.7 \\
\hline Centrolene hesperium & 12 & $25.9[55]$ & $1650[36]$ & 17.8 & 6.9 & 67.0 & 28.8 & 560.5 & 5779.0 \\
\hline Centrolene hybrida & 9 & $21.4[39]$ & $1715[36]$ & 18.5 & 3.6 & 79.5 & 40.9 & 2615.5 & 5021.0 \\
\hline Centrolene lynchi & 12 & $25.0[47,56-58]$ & $1487.5[36]$ & 18.4 & 2.4 & 79.1 & 47.8 & 2984.3 & 4512.6 \\
\hline Centrolene muelleri & 10 & $23.5[44]$ & 1915 [36] & 18.2 & 5.8 & 79.5 & 12.3 & 2505.1 & 5361.2 \\
\hline Centrolene peristictum & 7 & $19.9[43]$ & 1625 [57] & 19.2 & 3.2 & 68.8 & 52.9 & 1055.5 & 5390.6 \\
\hline Centrolene pipilatum & 9 & $21.3[43]$ & $1520[36]$ & 19.1 & 0.2 & 80.2 & 25.3 & 2240.8 & 5074.3 \\
\hline Centrolene savagei & 7 & $21.9[51]$ & $1905[36]$ & 18.4 & 4.6 & 81.2 & 47.4 & 2822.7 & 4840.4 \\
\hline
\end{tabular}




\begin{tabular}{|c|c|c|c|c|c|c|c|c|c|}
\hline Species & $\begin{array}{c}\text { Div. } \\
\text { events } \\
\text { (number) }\end{array}$ & $\begin{array}{l}\text { Midpoint Snout Vent } \\
\text { Length }(\mathrm{mm})\end{array}$ & $\begin{array}{c}\text { Midpoint } \\
\text { elevation }(m)\end{array}$ & $\begin{array}{l}\text { Mean annual } \\
\text { temperature } \\
\left({ }^{\circ} \mathrm{C}\right)\end{array}$ & $\begin{array}{l}\text { Midpoint } \\
\text { latitude } \\
\text { (degrees) }\end{array}$ & $\begin{array}{l}\text { Mean } \\
\text { annual } \\
\text { RH (\%) }\end{array}$ & $\begin{array}{l}\text { Mean annual } \\
\operatorname{NPP}\left(g C * 10^{9}\right)\end{array}$ & $\begin{array}{l}\text { Mean annual } \\
\text { precipitation } \\
(\mathrm{mm})\end{array}$ & $\begin{array}{c}\text { Mean } \\
\text { annual UVB } \\
\left(\mathrm{J} / \mathrm{m}^{2}\right)\end{array}$ \\
\hline Centrolene venezuelense & 11 & $28.6[40]$ & $2575[36]$ & 13.4 & 8.9 & 78.4 & 46.1 & 2310.3 & 5731.9 \\
\hline Chimerella corleone & 8 & 19.9 [unpub. data] & $\begin{array}{l}610 \text { [unpub. } \\
\text { data] }\end{array}$ & 24.8 & 6.4 & 76.0 & 95.5 & 1647.0 & 5308.0 \\
\hline Chimerella mariaelenae & 8 & $19.1[23,59]$ & $1610[36]$ & 19.3 & 2.4 & 74.2 & 28.4 & 1074.7 & 5159.8 \\
\hline Cochranella erminea & 10 & $23.2[7,60]$ & $\begin{array}{c}585[7,36 \\
\text { unpub. data] }\end{array}$ & 24.7 & 8.7 & 78.0 & 57.6 & 2683.8 & 5126.9 \\
\hline Cochranella euknemos & 11 & $26.5[61-63]$ & $870[36]$ & 24.3 & 7.7 & 76.1 & 96.0 & 1519.4 & 5209.3 \\
\hline Cochranella granulosa & 11 & $\begin{array}{c}27.5[47,51,61,64 \\
\text { unpub. data] }\end{array}$ & $760[36]$ & 24.6 & 10.7 & 84.1 & 54.9 & 4479.4 & 4918.3 \\
\hline Cochranella guayasamini & 10 & $24.8[44]$ & $818.5[36]$ & 23.9 & 6.6 & 77.7 & 52.1 & 1444.3 & 5464.8 \\
\hline Cochranella litoralis & 7 & $19.4[65]$ & $160[36]$ & 25.3 & 1.1 & 82.3 & 67.1 & 2664.8 & 4822.3 \\
\hline Cochranella mache & 11 & $23.8[64]$ & $372.5[36]$ & 24.5 & 0.7 & 76.8 & 39.1 & 2288.9 & 4616.1 \\
\hline Cochranella nola & 8 & $23.2[66,67]$ & $1125[36]$ & 21.3 & 16.1 & 65.5 & 87.4 & 1847.0 & 4990.7 \\
\hline Cochranella resplendens & 11 & $27.0[43]$ & $350[36]$ & 24.8 & 3.2 & 76.9 & 92.8 & 3104.9 & 5169.9 \\
\hline Espadarana andina & 10 & $25.5[35,40]$ & $1502.5[1,36]$ & 18.1 & 7.8 & 82.4 & 57.1 & 5941.4 & 4949.8 \\
\hline "Espadarana audax" & 9 & $24.2[43,44]$ & $1350[36]$ & 19.2 & 2.5 & 80.3 & 42.3 & 2513.3 & 5030.8 \\
\hline Espadarana callistomma & 10 & $29.3[68]$ & $260[8,36]$ & 26.1 & 2.9 & 81.5 & 64.7 & 3254.0 & 4826.1 \\
\hline "Espadarana durrellorum" & 9 & $25.9[52]$ & $685[36]$ & 23.7 & 2.2 & 78.3 & 91.0 & 3254.1 & 5135.9 \\
\hline Espadarana prosoblepon & 10 & $25.5[62,69]$ & $977.5[9,36]$ & 24.5 & 6.1 & 84.0 & 92.4 & 3223.0 & 5558.0 \\
\hline Espadarana sp & 10 & No data & $\begin{array}{l}1500 \text { [unpub. } \\
\text { data] }\end{array}$ & 20.3 & 7 & 82.8 & 49.7 & 4917.9 & 4985.9 \\
\hline Hyalinobatrachium aff bergeri & 7 & 23.1 [unpub. data] & $\begin{array}{l}1120 \text { [unpub. } \\
\text { data] }\end{array}$ & 21.5 & 15.1 & 70.3 & 49.8 & 3321.4 & 5117.9 \\
\hline Hyalinobatrachium aureoguttatum & 5 & $22.2[70]$ & $807.5[36]$ & 21.8 & 11 & 78.4 & 75.9 & 1623.6 & 5237.4 \\
\hline Hyalinobatrachium bergeri & 7 & $23.4[71]$ & $1150[20]$ & 24.9 & 4.3 & 79.5 & 97.8 & 2548.2 & 5669.5 \\
\hline Hyalinobatrachium cappellei & 6 & $21.8[13,35,72-74]$ & $1005[13]$ & 23.8 & 2.7 & 69.4 & 84.0 & 3363.4 & 4604.5 \\
\hline Hyalinobatrachium carlesvilai & 7 & $22.6[20]$ & $750[20]$ & 23.8 & 13.3 & 83.4 & 76.1 & 2733.3 & 4765.5 \\
\hline Hyalinobatrachium chirripoi & 8 & $25.7[61,62,75]$ & $300[36]$ & 27.5 & 10.7 & 83.1 & 68.0 & 3887.7 & 4978.5 \\
\hline
\end{tabular}




\begin{tabular}{|c|c|c|c|c|c|c|c|c|c|}
\hline Species & $\begin{array}{c}\text { Div. } \\
\text { events } \\
\text { (number) }\end{array}$ & $\begin{array}{l}\text { Midpoint Snout Vent } \\
\text { Length (mm) }\end{array}$ & $\begin{array}{c}\text { Midpoint } \\
\text { elevation }(m)\end{array}$ & $\begin{array}{c}\text { Mean annual } \\
\text { temperature } \\
\left({ }^{\circ} \mathrm{C}\right)\end{array}$ & $\begin{array}{l}\text { Midpoint } \\
\text { latitude } \\
\text { (degrees) }\end{array}$ & $\begin{array}{l}\text { Mean } \\
\text { annual } \\
\text { RH (\%) }\end{array}$ & $\begin{array}{l}\text { Mean annual } \\
\operatorname{NPP}\left(g C * 10^{9}\right)\end{array}$ & $\begin{array}{l}\text { Mean annual } \\
\text { precipitation } \\
(\mathrm{mm})\end{array}$ & $\begin{array}{c}\text { Mean } \\
\text { annual UVB } \\
\left(\mathrm{J} / \mathrm{m}^{2}\right)\end{array}$ \\
\hline Hyalinobatrachium colymbiphyllum & 8 & $25.6[61,62,76]$ & $905[36,53]$ & 25.2 & 9.4 & 73.2 & 22.1 & 1263.9 & 5928.1 \\
\hline Hyalinobatrachium duranti & 7 & $22.8[34,35]$ & $2100[36]$ & 15.5 & 8.6 & 79.4 & 74.0 & 2646.3 & 4989.4 \\
\hline Hyalinobatrachium fleischmanni & 8 & $25.5[69]$ & $845[15,36]$ & 24.7 & 9.3 & 77.0 & 70.2 & 1253.7 & 5172.3 \\
\hline Hyalinobatrachium fragile & 8 & $21.7[34,35]$ & $400[36]$ & 24.4 & 10.1 & 77.5 & 98.1 & 2514.4 & 5583.3 \\
\hline Hyalinobatrachium iaspidiense & 6 & $20.7[35,77,78]$ & $505[36]$ & 25.3 & 0 & 88.9 & 95.7 & 3764.2 & 5381.8 \\
\hline Hyalinobatrachium kawense & 7 & $20.0[13]$ & $5[36]$ & 26.1 & 4.6 & 75.2 & 104.3 & 2521.8 & 5730.2 \\
\hline Hyalinobatrachium mondolfii & 8 & $21.4[13,79]$ & $142.5[18]$ & 26.2 & 0.7 & 77.5 & 88.0 & 3082.1 & 5214.7 \\
\hline Hyalinobatrachium munozorum & 8 & $22.3[43]$ & $560[18,20]$ & 25.2 & 3.3 & 83.0 & 67.4 & 1416.5 & 5259.9 \\
\hline Hyalinobatrachium orientale & 7 & $21.7[35,40]$ & $695[36]$ & 22.1 & 10.6 & 76.6 & 70.8 & 1361.7 & 5456.6 \\
\hline Hyalinobatrachium orocostale & 8 & $21.4[19,40]$ & $1350[36]$ & 18.9 & 10 & 77.9 & 48.5 & 1996.8 & 6176.1 \\
\hline Hyalinobatrachium pallidum & 7 & $22.5[1,34,35,80]$ & $1591[36]$ & 18.6 & 8.2 & 79.6 & 49.9 & 2438.6 & 5043.0 \\
\hline Hyalinobatrachium pellucidum & 7 & $21.2[43,44]$ & $\begin{array}{l}1131.5 \\
{[20,36]}\end{array}$ & 20 & 6.4 & 83.6 & 51.1 & 3311.5 & 4521.8 \\
\hline Hyalinobatrachium talamancae & 5 & $24.0[61,62,81,82]$ & $758[36]$ & 22.7 & 9.1 & 76.0 & 85.9 & 1953.0 & 5316.8 \\
\hline Hyalinobatrachium tatayoi & 8 & $22.1[83]$ & $371[1,36]$ & 25.4 & 10.2 & 77.9 & 95.5 & 2493.5 & 5701.9 \\
\hline Hyalinobatrachium taylori & 6 & $19.7[35,84]$ & $940[36]$ & 23.5 & 4.2 & 89.8 & 101.3 & 3807.5 & 5360.8 \\
\hline Hyalinobatrachium tricolor & 6 & $20.7[13]$ & $76[13,36]$ & 25.8 & 4.5 & 80.9 & 57.7 & 4150.5 & 4808.1 \\
\hline Hyalinobatrachium valerioi & 5 & $22.8[61,62,85]$ & $705[57]$ & 25.1 & 3.9 & 79.9 & 47.3 & 2396.0 & 4862.0 \\
\hline Ikakogi tayrona & 0 & $30.9[39]$ & $1385[36]$ & 19.1 & 10.7 & 82.8 & 41.2 & 3718.4 & 4741.9 \\
\hline Nymphargus aff cochranae & 10 & No data & $\begin{array}{l}1960 \text { [unpub. } \\
\text { data] }\end{array}$ & 21.9 & 6.1 & 60.7 & 68.7 & 978.0 & 5363.9 \\
\hline Nymphargus anomalus & 10 & $24.1[43]$ & $1719[36]$ & 17.6 & 4 & 76.6 & 95.0 & 1384.7 & 4954.0 \\
\hline Nymphargus bejaranoi & 6 & $24.3[71]$ & $2000[36]$ & 18.3 & 0.7 & 75.5 & 48.7 & 1529.0 & 5921.0 \\
\hline Nymphargus chancas & 7 & $24.9[44]$ & $1170[36]$ & 18.3 & 17.7 & 79.4 & 57.8 & 3337.6 & 5041.2 \\
\hline Nymphargus cochranae & 8 & $30.0[38,86]$ & $1350[21,36]$ & 20.1 & 2.1 & 79.0 & 39.7 & 1914.8 & 5625.3 \\
\hline Nymphargus garciae & 7 & $26.0[87]$ & $2465[36]$ & 15 & 3 & 79.4 & 33.4 & 2226.3 & 5680.5 \\
\hline
\end{tabular}




\begin{tabular}{|c|c|c|c|c|c|c|c|c|c|}
\hline Species & $\begin{array}{c}\text { Div. } \\
\text { events } \\
\text { (number) }\end{array}$ & $\begin{array}{l}\text { Midpoint Snout Vent } \\
\text { Length (mm) }\end{array}$ & $\begin{array}{l}\text { Midpoint } \\
\text { elevation }(m)\end{array}$ & $\begin{array}{l}\text { Mean annual } \\
\text { temperature } \\
\left({ }^{\circ} \mathrm{C}\right)\end{array}$ & $\begin{array}{l}\text { Midpoint } \\
\text { latitude } \\
\text { (degrees) }\end{array}$ & $\begin{array}{l}\text { Mean } \\
\text { annual } \\
\text { RH (\%) }\end{array}$ & $\begin{array}{l}\text { Mean annual } \\
\operatorname{NPP}\left(g C * 10^{9}\right)\end{array}$ & $\begin{array}{l}\text { Mean annual } \\
\text { precipitation } \\
(\mathrm{mm})\end{array}$ & $\begin{array}{c}\text { Mean } \\
\text { annual UVB } \\
\left(\mathrm{J} / \mathrm{m}^{2}\right)\end{array}$ \\
\hline Nymphargus grandisonae & 6 & $27.9[56,88]$ & $1925[36]$ & 17.7 & 3.4 & 80.3 & 21.8 & 2266.9 & 5142.0 \\
\hline Nymphargus griffithsi & 10 & $23.2[38,43,86]$ & $2215[36]$ & 16.7 & 3.2 & 80.8 & 37.4 & 2450.8 & 5205.4 \\
\hline Nymphargus megacheirus & 10 & $30.0[43]$ & $1525[36]$ & 19.1 & 0.2 & 69.0 & 38.6 & 685.3 & 6067.9 \\
\hline Nymphargus mixomaculatus & 5 & $24.6[22]$ & $2687.5[36]$ & 15.2 & 9.4 & 75.8 & 75.5 & 1637.9 & 5745.4 \\
\hline Nymphargus ocellatus & 8 & $25.0[89,90]$ & $1450[36]$ & 21.1 & 11.9 & 63.1 & 65.7 & 1288.4 & 6388.4 \\
\hline Nymphargus pluvialis & 8 & $23.1[38,91]$ & $2035[36]$ & 18.7 & 14.4 & 80.2 & 49.6 & 2281.0 & 5516.5 \\
\hline Nymphargus posadae & 7 & $32.2[87]$ & $1950[36]$ & 16.9 & 2.5 & 79.0 & 60.6 & 4404.3 & 4975.2 \\
\hline Nymphargus puyoensis & 7 & $25.6[92]$ & $725[36]$ & 21.2 & 1.5 & 80.8 & 66.8 & 3420.8 & 5435.4 \\
\hline Nymphargus rosada & 8 & $26.8[41]$ & $1550[36]$ & 19.7 & 5.9 & 81.2 & 43.7 & 2520.3 & 4858.8 \\
\hline Nymphargus siren & 9 & $21.8[43]$ & $1505[36]$ & 19.2 & 0.1 & 73.0 & 57.9 & 1237.3 & 7557.0 \\
\hline Nymphargus vicenteruedai & 7 & $25.6[93]$ & $2675[36]$ & 13.4 & 6 & 83.0 & 39.8 & 2959.2 & 4447.0 \\
\hline Nymphargus wileyi & 9 & $25.2[38]$ & $2100[36]$ & 16.6 & 0.4 & 79.8 & 67.8 & 2652.9 & 5804.7 \\
\hline Rulyrana adiazeta & 7 & $25.2[87,94]$ & $1230[36]$ & 21.2 & 5.3 & 76.7 & 97.8 & 1684.9 & 5104.6 \\
\hline Rulyrana flavopunctata & 10 & 23.2 [43], [unpub. data] & $650[36]$ & 24 & 0.8 & 78.8 & 80.7 & 3547.0 & 5190.6 \\
\hline Rulyrana spiculata & 8 & $24.6[90,95]$ & $1450[36]$ & 21.2 & 12.1 & 74.9 & 80.7 & 1647.8 & 5857.3 \\
\hline Sachatamia albomaculata & 8 & $26.3[62,76]$ & $750[36]$ & 25.8 & 8 & 82.0 & 65.3 & 2813.2 & 4815.6 \\
\hline Sachatamia ilex & 8 & $30.7[61,62,68,96]$ & $735[35,36]$ & 25.1 & 5.6 & 84.0 & 69.4 & 4111.6 & 4806.8 \\
\hline Sachatamia orejuela & 8 & $30.5[25,47]$ & $875[36]$ & 22.5 & 2 & 80.3 & 20.7 & 3129.1 & 4524.7 \\
\hline Sachatamia punctulata & 8 & $28.1[87]$ & $715[36]$ & 24.7 & 5.5 & 77.1 & 93.5 & 2833.3 & 5956.8 \\
\hline Teratohyla adenocheira & 7 & $23.2[95]$ & $230[26,27]$ & 25.2 & 10.9 & 82.2 & 97.2 & 2148.0 & 5192.9 \\
\hline Teratohyla amelie & 6 & $18.5[97]$ & $474.5[36,98]$ & 24 & 7.3 & 75.0 & 96.8 & 3321.8 & 5242.9 \\
\hline Teratohyla midas & 7 & $21.5[43]$ & $395[21,36]$ & 25.4 & 3.7 & 79.7 & 96.7 & 2348.3 & 5126.1 \\
\hline Teratohyla pulverata & 6 & $27.5[62,99]$ & $426[36]$ & 25.3 & 7.8 & 82.1 & 71.4 & 3405.7 & 4829.6 \\
\hline Teratohyla spinosa & 6 & $20.4[62,76]$ & $401[36,53]$ & 25.5 & 7.1 & 80.8 & 65.5 & 4256.2 & 4780.9 \\
\hline Vitreorana antisthenesi & 5 & $24.2[35,100]$ & $710[36]$ & 22.6 & 10.2 & 77.3 & 61.5 & 1140.4 & 5109.1 \\
\hline
\end{tabular}




\begin{tabular}{|c|c|c|c|c|c|c|c|c|c|}
\hline Species & $\begin{array}{c}\text { Div. } \\
\text { events } \\
\text { (number) }\end{array}$ & $\begin{array}{l}\text { Midpoint Snout Vent } \\
\text { Length }(\mathrm{mm})\end{array}$ & $\begin{array}{l}\text { Midpoint } \\
\text { elevation }(\mathrm{m})\end{array}$ & $\begin{array}{l}\text { Mean annual } \\
\text { temperature } \\
\left({ }^{\circ} \mathrm{C}\right)\end{array}$ & $\begin{array}{l}\text { Midpoint } \\
\text { latitude } \\
\text { (degrees) }\end{array}$ & $\begin{array}{l}\text { Mean } \\
\text { annual } \\
\text { RH (\%) }\end{array}$ & $\begin{array}{l}\text { Mean annual } \\
\operatorname{NPP}\left(g C * 10^{9}\right)\end{array}$ & $\begin{array}{l}\text { Mean annual } \\
\text { precipitation } \\
(\mathrm{mm})\end{array}$ & $\begin{array}{c}\text { Mean } \\
\text { annual UVB } \\
\left(\mathrm{J} / \mathrm{m}^{2}\right)\end{array}$ \\
\hline Vitreorana castroviejoi & 5 & $23.2[37]$ & $690[36]$ & 22.3 & 10.7 & 79.2 & 40.9 & 1943.3 & 5440.4 \\
\hline Vitreorana eurygnatha & 6 & $21.0[101,102]$ & $900[36,103]$ & 19.7 & 20.4 & 77.7 & 77.1 & 1419.8 & 4335.9 \\
\hline Vitreorana gorzulae & 6 & $20.4[77]$ & $1175[29,36]$ & 21.4 & 5.6 & 78.0 & 96.0 & 2141.8 & 5697.6 \\
\hline Vitreorana helenae & 7 & $19.8[35,77]$ & $715[36]$ & 22.5 & 5.3 & 77.9 & 82.6 & 2313.2 & 5805.3 \\
\hline Vitreorana oyampiensis & 7 & $20.5[104]$ & $205[36]$ & 26.2 & 2.7 & 79.7 & 101.6 & 2346.2 & 5729.8 \\
\hline Vitreorana uranoscopa & 6 & $22.8[102,105]$ & $934.5[30,36]$ & 18.5 & 23.6 & 76.5 & 78.3 & 1520.3 & 4256.7 \\
\hline
\end{tabular}




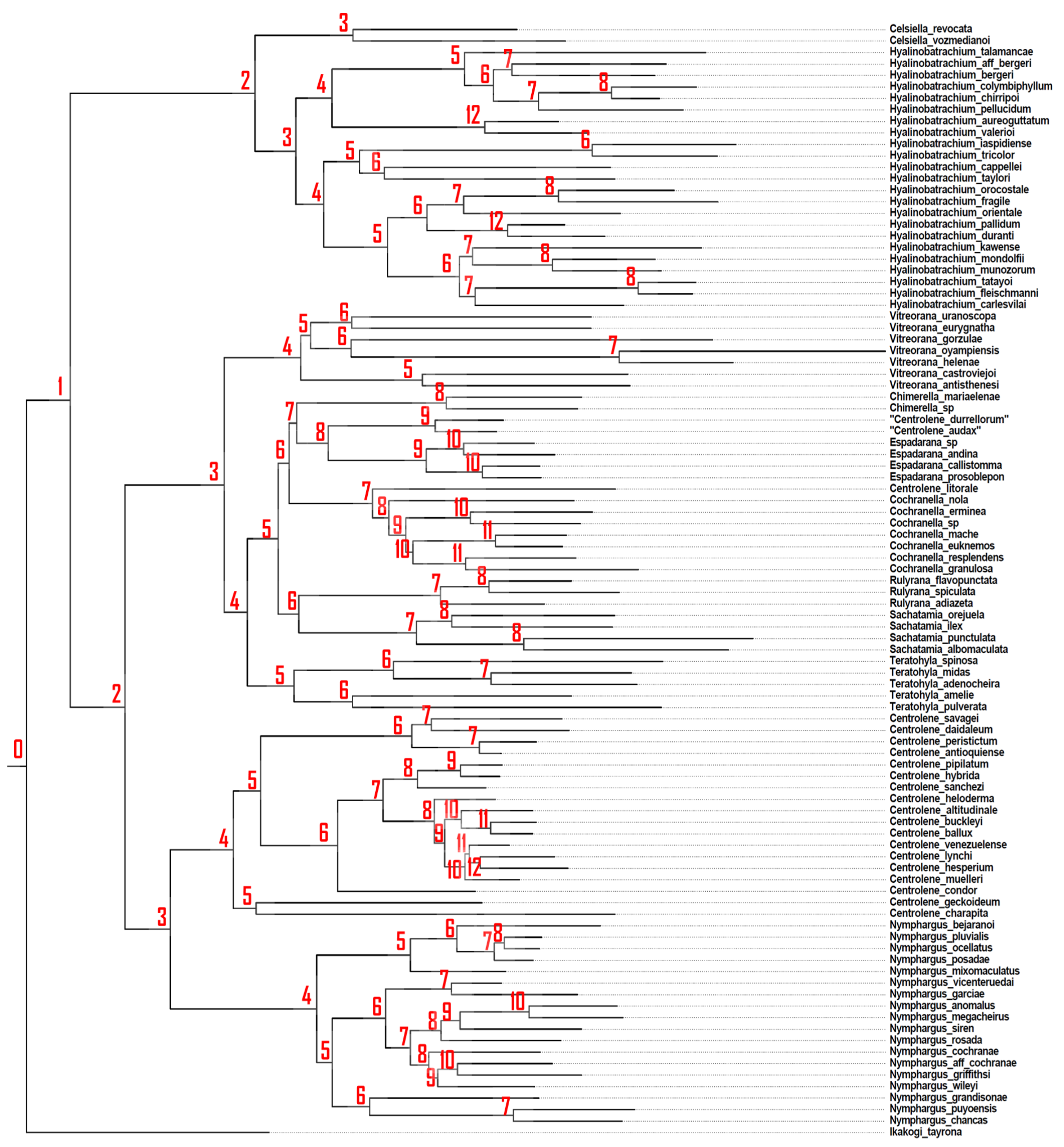


Appendix S5. Minimum effective size and maximum discrepancy were obtained from the tracecomp module of Coevol for all parameters for each pair of independent analyses to assess convergence. A minimum effective size of 300 and maximum discrepancy of 0.1 are necessary for a run to be considered as good. If the minimum effective size is 50 and maximum discrepancy is below 0.3 a run is considered acceptable (yielding qualitatively correct results).

\begin{tabular}{|c|c|c|c|c|c|}
\hline \multicolumn{6}{|c|}{ MITOCHONDRIAL NON-CODING GENES } \\
\hline Parameters & Effective size & Discrepancy & Parameters & Effective size & Discrepancy \\
\hline \#logprior & 4936 & 0.005 & sigma_6_9 & 4997 & 0.009 \\
\hline $\operatorname{lnL}$ & 4025 & 0.017 & sigma_6_10 & 3433 & 0.021 \\
\hline length & 5097 & 0.002 & sigma_7_8 & 5620 & 0.011 \\
\hline rootage & 5620 & 0.002 & sigma_7_9 & 5397 & 0.002 \\
\hline sigma_1_2 & 5620 & 0.019 & sigma_7_10 & 5620 & 0.013 \\
\hline sigma_1_3 & 3937 & 0.010 & sigma_8_9 & 5620 & 0.007 \\
\hline sigma_1_4 & 5147 & 0.000 & sigma_8_10 & 5620 & 0.010 \\
\hline sigma_1_5 & 5497 & 0.038 & sigma_9_10 & 5593 & 0.006 \\
\hline sigma_1_6 & 5620 & 0.024 & sigma_1_1 & 5620 & 0.014 \\
\hline sigma_1_7 & 5620 & 0.000 & sigma_2_2 & 5620 & 0.017 \\
\hline sigma_1_8 & 4515 & 0.023 & sigma_3_3 & 5620 & 0.006 \\
\hline sigma_1_9 & 4357 & 0.008 & sigma_4_4 & 3209 & 0.009 \\
\hline sigma_1_10 & 4647 & 0.034 & sigma_5_5 & 4447 & 0.021 \\
\hline sigma_2_3 & 5365 & 0.006 & sigma_6_6 & 5620 & 0.019 \\
\hline sigma_2_4 & 5620 & 0.005 & sigma_7_7 & 5289 & 0.005 \\
\hline sigma_2_5 & 4979 & 0.011 & sigma_8_8 & 5620 & 0.007 \\
\hline sigma_2_6 & 5347 & 0.003 & sigma_9_9 & 5620 & 0.025 \\
\hline sigma_2_7 & 5273 & 0.027 & sigma_10_10 & 3769 & 0.009 \\
\hline sigma_2_8 & 5620 & 0.043 & $\operatorname{dim}$ & 5620 & 0.000 \\
\hline sigma_2_9 & 5620 & 0.015 & root_1 & 2392 & 0.048 \\
\hline sigma_2_10 & 5620 & 0.014 & root_2 & 3865 & 0.022 \\
\hline sigma_3_4 & 5620 & 0.032 & root_3 & 4409 & 0.040 \\
\hline sigma_3_5 & 5620 & 0.034 & root_4 & 5620 & 0.044 \\
\hline sigma_3_6 & 5620 & 0.010 & root_5 & 5620 & 0.036 \\
\hline sigma_3_7 & 5620 & 0.026 & root_6 & 4071 & 0.046 \\
\hline sigma_3_8 & 5620 & 0.003 & root_7 & 5085 & 0.009 \\
\hline sigma_3_9 & 5620 & 0.013 & root_8 & 3577 & 0.010 \\
\hline sigma_3_10 & 5620 & 0.009 & root_9 & 3993 & 0.046 \\
\hline sigma_4_5 & 5620 & 0.020 & root_10 & 5620 & 0.035 \\
\hline sigma_4_6 & 4085 & 0.007 & statent & 5620 & 0.019 \\
\hline sigma_4_7 & 5152 & 0.010 & rrent & 4734 & 0.008 \\
\hline sigma_4_8 & 3768 & 0.003 & diag0 & 5620 & 0.006 \\
\hline sigma_4_9 & 4953 & 0.003 & diag1 & 5620 & 0.011 \\
\hline sigma_4_10 & 5620 & 0.017 & diag2 & 4191 & 0.004 \\
\hline sigma_5_6 & 5620 & 0.035 & diag3 & 5620 & 0.001 \\
\hline sigma_5_7 & 5620 & 0.008 & diag4 & 5620 & 0.008 \\
\hline sigma_5_8 & 5107 & 0.008 & diag5 & 5620 & 0.003 \\
\hline sigma_5_9 & 5620 & 0.009 & diag6 & 4056 & 0.007 \\
\hline sigma_5_10 & 4913 & 0.010 & diag7 & 4178 & 0.031 \\
\hline sigma_6_7 & 5620 & 0.000 & diag8 & 5620 & 0.018 \\
\hline sigma_6_8 & 3424 & 0.003 & diag9 & 5620 & 0.008 \\
\hline
\end{tabular}




\begin{tabular}{|c|c|c|c|c|c|}
\hline \multicolumn{6}{|c|}{ MITOCHONDRIAL CODING GENE (dN) } \\
\hline Parameters & Effective size & Discrepancy & Parameters & Effective size & Discrepancy \\
\hline \#logprior & 4264 & 0.072 & sigma_6_10 & 10037 & 0.001 \\
\hline $\ln L$ & 3460 & 0.003 & sigma_6_11 & 8261 & 0.004 \\
\hline length & 2442 & 0.029 & sigma_7_8 & 9707 & 0.013 \\
\hline $\mathrm{dN}$ & 2520 & 0.018 & sigma_7_9 & 13720 & 0.026 \\
\hline rootage & 13086 & 0.014 & sigma_7_10 & 8716 & 0.018 \\
\hline sigma_1_2 & 4651 & 0.000 & sigma_7_11 & 11351 & 0.001 \\
\hline sigma_1_3 & 8077 & 0.034 & sigma_8_9 & 5479 & 0.021 \\
\hline sigma_1_4 & 13720 & 0.016 & sigma_8_10 & 5877 & 0.007 \\
\hline sigma_1_5 & 13157 & 0.032 & sigma_8_11 & 8082 & 0.025 \\
\hline sigma_1_6 & 12836 & 0.005 & sigma_9_10 & 10674 & 0.001 \\
\hline sigma_1_7 & 5023 & 0.025 & sigma_9_11 & 8667 & 0.015 \\
\hline sigma_1_8 & 11226 & 0.005 & sigma_10_11 & 11177 & 0.003 \\
\hline sigma_1_9 & 2322 & 0.062 & sigma_1_1 & 3921 & 0.011 \\
\hline sigma_1_10 & 5440 & 0.023 & sigma_2_2 & 4928 & 0.032 \\
\hline sigma_1_11 & 2722 & 0.013 & sigma_3_3 & 13720 & 0.014 \\
\hline sigma_2_3 & 8259 & 0.006 & sigma_4_4 & 9981 & 0.017 \\
\hline sigma_2_4 & 8334 & 0.022 & sigma_5_5 & 7120 & 0.041 \\
\hline sigma_2_5 & 2161 & 0.006 & sigma_6_6 & 2154 & 0.035 \\
\hline sigma_2_6 & 10131 & 0.005 & sigma_7_7 & 10893 & 0.018 \\
\hline sigma_2_7 & 2397 & 0.010 & sigma_8_8 & 4128 & 0.042 \\
\hline sigma_2_8 & 8029 & 0.024 & sigma_9_9 & 5593 & 0.037 \\
\hline sigma_2_9 & 3887 & 0.015 & sigma_10_10 & 3657 & 0.006 \\
\hline sigma_2_10 & 4832 & 0.020 & sigma_11_11 & 7281 & 0.029 \\
\hline sigma_2_11 & 4383 & 0.019 & $\operatorname{dim}$ & 13720 & 0.000 \\
\hline sigma_3_4 & 13720 & 0.024 & root_1 & 3693 & 0.026 \\
\hline sigma_3_5 & 8280 & 0.017 & root_2 & 7888 & 0.012 \\
\hline sigma_3_6 & 13720 & 0.026 & root_3 & 2514 & 0.059 \\
\hline sigma_3_7 & 13720 & 0.008 & root_4 & 8321 & 0.005 \\
\hline sigma_3_8 & 9793 & 0.008 & root_5 & 8855 & 0.013 \\
\hline sigma_3_9 & 13720 & 0.012 & root_6 & 3853 & 0.053 \\
\hline sigma_3_10 & 12518 & 0.004 & root_7 & 8231 & 0.034 \\
\hline sigma_3_11 & 13720 & 0.029 & root_8 & 11551 & 0.003 \\
\hline sigma_4_5 & 10365 & 0.001 & root_9 & 4285 & 0.003 \\
\hline sigma_4_6 & 13720 & 0.014 & root_10 & 8597 & 0.021 \\
\hline sigma_4_7 & 9247 & 0.015 & root_11 & 11891 & 0.022 \\
\hline sigma_4_8 & 13720 & 0.002 & statent & 989 & 0.063 \\
\hline sigma_4_9 & 13720 & 0.009 & rrent & 469 & 0.085 \\
\hline sigma_4_10 & 13720 & 0.004 & diag0 & 5625 & 0.028 \\
\hline sigma_4_11 & 13720 & 0.016 & diag1 & 3885 & 0.041 \\
\hline sigma_5_6 & 11366 & 0.009 & diag2 & 13720 & 0.010 \\
\hline sigma_5_7 & 13720 & 0.015 & diag3 & 13720 & 0.006 \\
\hline sigma_5_8 & 8263 & 0.008 & diag4 & 10555 & 0.009 \\
\hline sigma_5_9 & 13720 & 0.022 & diag5 & 13150 & 0.005 \\
\hline sigma_5_10 & 11790 & 0.030 & diag6 & 5869 & 0.023 \\
\hline sigma_5_11 & 12086 & 0.006 & diag7 & 8586 & 0.032 \\
\hline sigma_6_7 & 12649 & 0.007 & diag8 & 13720 & 0.022 \\
\hline sigma_6_8 & 13720 & 0.013 & diag9 & 13720 & 0.014 \\
\hline sigma_6_9 & 8595 & 0.022 & $\operatorname{diag} 10$ & 10484 & 0.025 \\
\hline
\end{tabular}




\begin{tabular}{|c|c|c|c|c|c|}
\hline \multicolumn{6}{|c|}{ MITOCHONDRIAL CODING GENE $(\omega)$} \\
\hline Parameters & Effective size & Discrepancy & Parameters & Effective size & Discrepancy \\
\hline \#logprior & 466 & 0.082 & sigma_6_10 & 8497 & 0.003 \\
\hline $\operatorname{lnL}$ & 3353 & 0.018 & sigma_6_11 & 7180 & 0.017 \\
\hline length & 750 & 0.027 & sigma_7_8 & 6206 & 0.029 \\
\hline$\omega$ & 1799 & 0.002 & sigma_7_9 & 8410 & 0.012 \\
\hline rootage & 9158 & 0.001 & sigma_7_10 & 9163 & 0.027 \\
\hline sigma_1_2 & 6065 & 0.025 & sigma_7_11 & 7771 & 0.004 \\
\hline sigma_1_3 & 5212 & 0.052 & sigma_8_9 & 10839 & 0.017 \\
\hline sigma_1_4 & 6266 & 0.045 & sigma_8_10 & 6105 & 0.034 \\
\hline sigma_1_5 & 7551 & 0.004 & sigma_8_11 & 8899 & 0.030 \\
\hline sigma_1_6 & 2161 & 0.029 & sigma_9_10 & 10839 & 0.027 \\
\hline sigma_1_7 & 3200 & 0.015 & sigma_9_11 & 10229 & 0.033 \\
\hline sigma_1_8 & 7094 & 0.052 & sigma_10_11 & 7174 & 0.030 \\
\hline sigma_1_9 & 2565 & 0.045 & sigma_1_1 & 670 & 0.004 \\
\hline sigma_1_10 & 6043 & 0.097 & sigma_2_2 & 2777 & 0.017 \\
\hline sigma_1_11 & 6253 & 0.014 & sigma_3_3 & 8236 & 0.002 \\
\hline sigma_2_3 & 5474 & 0.030 & sigma_4_4 & 9312 & 0.038 \\
\hline sigma_2_4 & 3511 & 0.011 & sigma_5_5 & 9393 & 0.026 \\
\hline sigma_2_5 & 8200 & 0.009 & sigma_6_6 & 5015 & 0.011 \\
\hline sigma_2_6 & 2254 & 0.007 & sigma_7_7 & 8662 & 0.036 \\
\hline sigma_2_7 & 3399 & 0.012 & sigma_8_8 & 8937 & 0.037 \\
\hline sigma_2_8 & 4666 & 0.046 & sigma_9_9 & 7661 & 0.004 \\
\hline sigma_2_9 & 4229 & 0.003 & sigma_10_10 & 9344 & 0.065 \\
\hline sigma_2_10 & 7253 & 0.002 & sigma_11_11 & 8962 & 0.028 \\
\hline sigma_2_11 & 5239 & 0.028 & $\operatorname{dim}$ & 10839 & 0.000 \\
\hline sigma_3_4 & 10839 & 0.000 & root_1 & 2277 & 0.026 \\
\hline sigma_3_5 & 10839 & 0.013 & root_2 & 1332 & 0.002 \\
\hline sigma_3_6 & 7830 & 0.001 & root_3 & 10839 & 0.000 \\
\hline sigma_3_7 & 9707 & 0.008 & root_4 & 9310 & 0.032 \\
\hline sigma_3_8 & 10839 & 0.016 & root_5 & 4104 & 0.020 \\
\hline sigma_3_9 & 10839 & 0.014 & root_6 & 5364 & 0.014 \\
\hline sigma_3_10 & 8179 & 0.021 & root_7 & 9228 & 0.024 \\
\hline sigma_3_11 & 10839 & 0.006 & root_8 & 10839 & 0.013 \\
\hline sigma_4_5 & 10839 & 0.003 & root_9 & 9842 & 0.043 \\
\hline sigma_4_6 & 7332 & 0.024 & root_10 & 7989 & 0.027 \\
\hline sigma_4_7 & 7929 & 0.023 & root_11 & 7474 & 0.008 \\
\hline sigma_4_8 & 9691 & 0.036 & statent & 864 & 0.042 \\
\hline sigma_4_9 & 10839 & 0.013 & rrent & 3726 & 0.023 \\
\hline sigma_4_10 & 10443 & 0.032 & diag0 & 1665 & 0.020 \\
\hline sigma_4_11 & 9234 & 0.027 & diag1 & 2010 & 0.012 \\
\hline sigma_5_6 & 10839 & 0.021 & diag2 & 10839 & 0.001 \\
\hline sigma_5_7 & 10839 & 0.021 & diag3 & 10839 & 0.033 \\
\hline sigma_5_8 & 6960 & 0.014 & diag4 & 9710 & 0.001 \\
\hline sigma_5_9 & 7631 & 0.004 & diag5 & 9358 & 0.000 \\
\hline sigma_5_10 & 10839 & 0.001 & diag6 & 7616 & 0.043 \\
\hline sigma_5_11 & 10839 & 0.043 & diag7 & 10839 & 0.029 \\
\hline sigma_6_7 & 10839 & 0.000 & diag8 & 10839 & 0.003 \\
\hline sigma_6_8 & 10839 & 0.006 & diag9 & 10839 & 0.008 \\
\hline sigma_6_9 & 10839 & 0.034 & $\operatorname{diag} 10$ & 10839 & 0.001 \\
\hline
\end{tabular}




\begin{tabular}{|c|c|c|c|c|c|}
\hline \multicolumn{6}{|c|}{ NUCLEAR CODING GENES (dN) } \\
\hline Parameters & Effective size & Discrepancy & Parameters & Effective size & Discrepancy \\
\hline \#logprior & 224 & 0.029 & sigma_6_10 & 23387 & 0.014 \\
\hline $\ln L$ & 3389 & 0.015 & sigma_6_11 & 8329 & 0.008 \\
\hline length & 3265 & 0.018 & sigma_7_8 & 11785 & 0.010 \\
\hline $\mathrm{dN}$ & 1249 & 0.034 & sigma_7_9 & 11260 & 0.009 \\
\hline rootage & 32825 & 0.017 & sigma_7_10 & 10472 & 0.010 \\
\hline sigma_1_2 & 1995 & 0.016 & sigma_7_11 & 10842 & 0.011 \\
\hline sigma_1_3 & 15184 & 0.013 & sigma_8_9 & 11271 & 0.011 \\
\hline sigma_1_4 & 9715 & 0.019 & sigma_8_10 & 10583 & 0.011 \\
\hline sigma_1_5 & 21586 & 0.034 & sigma_8_11 & 10593 & 0.012 \\
\hline sigma_1_6 & 12264 & 0.025 & sigma_9_10 & 10074 & 0.009 \\
\hline sigma_1_7 & 13985 & 0.010 & sigma_9_11 & 10992 & 0.012 \\
\hline sigma_1_8 & 13923 & 0.018 & sigma_10_11 & 11354 & 0.012 \\
\hline sigma_1_9 & 21813 & 0.013 & sigma_1_1 & 1995 & 0.012 \\
\hline sigma_1_10 & 8605 & 0.004 & sigma_2_2 & 1070 & 0.005 \\
\hline sigma_1_11 & 18320 & 0.022 & sigma_3_3 & 11883 & 0.011 \\
\hline sigma_2_3 & 13804 & 0.016 & sigma_4_4 & 12930 & 0.009 \\
\hline sigma_2_4 & 8581 & 0.017 & sigma_5_5 & 16417 & 0.010 \\
\hline sigma_2_5 & 6044 & 0.019 & sigma_6_6 & 25920 & 0.001 \\
\hline sigma_2_6 & 7645 & 0.010 & sigma_7_7 & 13751 & 0.007 \\
\hline sigma_2_7 & 17944 & 0.014 & sigma_8_8 & 11570 & 0.011 \\
\hline sigma_2_8 & 14813 & 0.019 & sigma_9_9 & 11923 & 0.007 \\
\hline sigma_2_9 & 21543 & 0.017 & sigma_10_10 & 11747 & 0.004 \\
\hline sigma_2_10 & 18876 & 0.025 & sigma_11_11 & 10394 & 0.014 \\
\hline sigma_2_11 & 12176 & 0.020 & $\operatorname{dim}$ & 36746 & 0.000 \\
\hline sigma_3_4 & 10952 & 0.013 & root_1 & 4347 & 0.001 \\
\hline sigma_3_5 & 10005 & 0.010 & root_2 & 1227 & 0.041 \\
\hline sigma_3_6 & 9868 & 0.006 & root_3 & 9191 & 0.022 \\
\hline sigma_3_7 & 9676 & 0.008 & root_4 & 11335 & 0.018 \\
\hline sigma_3_8 & 10123 & 0.009 & root_5 & 16922 & 0.025 \\
\hline sigma_3_9 & 10380 & 0.009 & root_6 & 6626 & 0.012 \\
\hline sigma_3_10 & 9948 & 0.010 & root_7 & 24272 & 0.008 \\
\hline sigma_3_11 & 10853 & 0.012 & root_8 & 16795 & 0.005 \\
\hline sigma_4_5 & 9147 & 0.011 & root_9 & 26382 & 0.009 \\
\hline sigma_4_6 & 10870 & 0.009 & root_10 & 20193 & 0.007 \\
\hline sigma_4_7 & 14335 & 0.008 & root_11 & 18903 & 0.012 \\
\hline sigma_4_8 & 11436 & 0.011 & statent & 30168 & 0.006 \\
\hline sigma_4_9 & 12659 & 0.011 & rrent & 2112 & 0.011 \\
\hline sigma_4_10 & 12735 & 0.012 & diag0 & 2015 & 0.032 \\
\hline sigma_4_11 & 11465 & 0.013 & diag1 & 536 & 0.020 \\
\hline sigma_5_6 & 12024 & 0.005 & diag2 & 36291 & 0.006 \\
\hline sigma_5_7 & 14218 & 0.009 & diag3 & 29354 & 0.017 \\
\hline sigma_5_8 & 9975 & 0.011 & diag4 & 17520 & 0.010 \\
\hline sigma_5_9 & 14682 & 0.010 & diag5 & 36746 & 0.003 \\
\hline sigma_5_10 & 22888 & 0.017 & diag6 & 9711 & 0.002 \\
\hline sigma_5_11 & 8531 & 0.014 & diag7 & 33620 & 0.007 \\
\hline sigma_6_7 & 12096 & 0.003 & diag8 & 31775 & 0.028 \\
\hline sigma_6_8 & 9834 & 0.007 & diag9 & 31721 & 0.004 \\
\hline sigma_6_9 & 18305 & 0.004 & $\operatorname{diag} 10$ & 31227 & 0.007 \\
\hline
\end{tabular}




\begin{tabular}{|c|c|c|c|c|c|}
\hline \multicolumn{6}{|c|}{ NUCLEAR CODING GENES $(\omega)$} \\
\hline Parameters & Effective size & Discrepancy & Parameters & Effective size & Discrepancy \\
\hline \#logprior & 415 & 0.091 & sigma_6_10 & 5379 & 0.036 \\
\hline $\ln L$ & 1910 & 0.022 & sigma_6_11 & 5369 & 0.031 \\
\hline length & 1714 & 0.019 & sigma_7_8 & 7576 & 0.024 \\
\hline$\omega$ & 1262 & 0.018 & sigma_7_9 & 5197 & 0.027 \\
\hline rootage & 7576 & 0.018 & sigma_7_10 & 5409 & 0.039 \\
\hline sigma_1_2 & 2669 & 0.008 & sigma_7_11 & 5655 & 0.022 \\
\hline sigma_1_3 & 1273 & 0.019 & sigma_8_9 & 3256 & 0.031 \\
\hline sigma_1_4 & 3224 & 0.052 & sigma_8_10 & 7576 & 0.004 \\
\hline sigma_1_5 & 7504 & 0.002 & sigma_8_11 & 5594 & 0.040 \\
\hline sigma_1_6 & 900 & 0.063 & sigma_9_10 & 2614 & 0.017 \\
\hline sigma_1_7 & 1688 & 0.008 & sigma_9_11 & 4896 & 0.000 \\
\hline sigma_1_8 & 2819 & 0.001 & sigma_10_11 & 4436 & 0.009 \\
\hline sigma_1_9 & 1217 & 0.047 & sigma_1_1 & 2204 & 0.048 \\
\hline sigma_1_10 & 1606 & 0.078 & sigma_2_2 & 1760 & 0.037 \\
\hline sigma_1_11 & 2789 & 0.033 & sigma_3_3 & 4627 & 0.002 \\
\hline sigma_2_3 & 940 & 0.009 & sigma_4_4 & 6938 & 0.025 \\
\hline sigma_2_4 & 4186 & 0.047 & sigma_5_5 & 4775 & 0.022 \\
\hline sigma_2_5 & 1250 & 0.046 & sigma_6_6 & 6735 & 0.029 \\
\hline sigma_2_6 & 2839 & 0.056 & sigma_7_7 & 5565 & 0.021 \\
\hline sigma_2_7 & 956 & 0.015 & sigma_8_8 & 6290 & 0.002 \\
\hline sigma_2_8 & 3552 & 0.040 & sigma_9_9 & 3457 & 0.014 \\
\hline sigma_2_9 & 1920 & 0.010 & sigma_10_10 & 5310 & 0.033 \\
\hline sigma_2_10 & 3551 & 0.026 & sigma_11_11 & 6219 & 0.021 \\
\hline sigma_2_11 & 3100 & 0.017 & $\operatorname{dim}$ & 7576 & 0.000 \\
\hline sigma_3_4 & 5805 & 0.021 & root_1 & 2016 & 0.015 \\
\hline sigma_3_5 & 5440 & 0.032 & root_2 & 5602 & 0.052 \\
\hline sigma_3_6 & 2700 & 0.001 & root_3 & 4413 & 0.004 \\
\hline sigma_3_7 & 5077 & 0.016 & root_4 & 5336 & 0.004 \\
\hline sigma_3_8 & 6696 & 0.014 & root_5 & 6844 & 0.043 \\
\hline sigma_3_9 & 3153 & 0.033 & root_6 & 6592 & 0.020 \\
\hline sigma_3_10 & 3500 & 0.021 & root_7 & 5262 & 0.057 \\
\hline sigma_3_11 & 3732 & 0.014 & root_8 & 7576 & 0.006 \\
\hline sigma_4_5 & 6301 & 0.018 & root_9 & 5279 & 0.036 \\
\hline sigma_4_6 & 4806 & 0.020 & root_10 & 7576 & 0.024 \\
\hline sigma_4_7 & 6351 & 0.001 & root_11 & 6774 & 0.013 \\
\hline sigma_4_8 & 7176 & 0.009 & statent & 5934 & 0.006 \\
\hline sigma_4_9 & 6003 & 0.027 & rrent & 888 & 0.046 \\
\hline sigma_4_10 & 3496 & 0.020 & diag0 & 2767 & 0.044 \\
\hline sigma_4_11 & 6090 & 0.037 & diag1 & 2000 & 0.029 \\
\hline sigma_5_6 & 7027 & 0.003 & diag2 & 7576 & 0.023 \\
\hline sigma_5_7 & 5002 & 0.039 & diag3 & 7576 & 0.015 \\
\hline sigma_5_8 & 5528 & 0.030 & diag4 & 7576 & 0.022 \\
\hline sigma_5_9 & 7576 & 0.039 & diag5 & 7576 & 0.009 \\
\hline sigma_5_10 & 6712 & 0.024 & diag6 & 2419 & 0.025 \\
\hline sigma_5_11 & 5504 & 0.032 & diag7 & 5942 & 0.008 \\
\hline sigma_6_7 & 5371 & 0.009 & diag8 & 7541 & 0.008 \\
\hline sigma_6_8 & 4115 & 0.015 & diag9 & 7576 & 0.004 \\
\hline sigma_6_9 & 4613 & 0.039 & $\operatorname{diag} 10$ & 7576 & 0.009 \\
\hline
\end{tabular}


Appendix S6. Number of cycles, burnin and read points per sequence analysed in Coevol.

\begin{tabular}{|c|c|c|c|}
\hline Analysis & \# Cycles & Burnin & $\begin{array}{l}\text { Read } \\
\text { points }\end{array}$ \\
\hline \multicolumn{4}{|l|}{ Mitochondrial } \\
\hline Non-coding 1 & 6800 & \multirow{2}{*}{1000} & 5800 \\
\hline Non-coding 2 & 6762 & & 5762 \\
\hline Coding $(d N) 1$ & 15720 & \multirow{2}{*}{2000} & 13720 \\
\hline Coding $(d N) 2$ & 15724 & & 13724 \\
\hline Coding $(\omega) 1$ & 14439 & \multirow{2}{*}{3600} & 10839 \\
\hline Coding $(\omega) 2$ & 14442 & & 10842 \\
\hline \multicolumn{4}{|l|}{ Nuclear } \\
\hline Coding $(d N) 1$ & 46791 & \multirow{2}{*}{10000} & 7358 \\
\hline Coding $(d N) 2$ & 46746 & & 7349 \\
\hline Coding $(\omega) 1$ & 12643 & \multirow{2}{*}{5000} & 7643 \\
\hline Coding $(\omega) 2$ & 12576 & & 7576 \\
\hline
\end{tabular}


Appendix S7. Results of the Coevol analyses. The tables show the covariances, correlation coefficients (Correl. Coeff.), posterior probabilities (Post. Prob.) and precisions, together with partial correlation coefficients (Partial C.C.) and their posterior probabilities, between all evaluated factors for each analysis: substitution rate (Subst. rate), rate of synonymous substitution (dS), rate of non-synonymous substitution $(\mathrm{dN})$ and the ratio of non-synonymous to synonymous substitution ( $\omega)$, diversification events (Diversif), body size, elevation (Elev), latitude (Lat), temperature (Temp), relative humidity $(\mathrm{RH})$, net primary productivity (NPP), precipitation (Prec) and UVB radiation (UVB). The tables also show the results when controlling for temperature.

\begin{tabular}{|c|c|c|c|c|c|c|c|c|c|c|}
\hline \multicolumn{11}{|c|}{ MITOHONDRIAL NON-CODING GENES: ANALYSIS 1} \\
\hline Covariances & $\begin{array}{c}\text { Subst. } \\
\text { rate }\end{array}$ & Diversif & Body size & Elev & Lat & Temp & HR & NPP & Prec & UVB \\
\hline Subst. rate & 0.45 & 0.06 & -0.03 & -0.31 & -0.12 & 0.07 & 0.00 & 0.06 & -0.01 & 0.00 \\
\hline Diversif & 0.06 & 0.08 & -0.01 & -0.01 & -0.06 & 0.00 & 0.00 & -0.01 & 0.01 & 0.00 \\
\hline Body size & -0.03 & -0.01 & 0.05 & 0.03 & 0.02 & 0.00 & 0.00 & 0.00 & 0.01 & 0.00 \\
\hline Elev & -0.31 & -0.01 & 0.03 & 1.46 & 0.26 & -0.12 & -0.03 & -0.12 & -0.16 & 0.02 \\
\hline Lat & -0.12 & -0.06 & 0.02 & 0.26 & 4.13 & -0.04 & 0.00 & 0.28 & -0.19 & 0.03 \\
\hline Temp & 0.07 & 0.00 & 0.00 & -0.12 & -0.04 & 0.03 & 0.00 & 0.03 & 0.04 & -0.01 \\
\hline$H R$ & 0.00 & 0.00 & 0.00 & -0.03 & 0.00 & 0.00 & 0.01 & -0.01 & 0.03 & 0.00 \\
\hline NPP & 0.06 & -0.01 & 0.00 & -0.12 & 0.28 & 0.03 & -0.01 & 0.43 & -0.02 & 0.02 \\
\hline Prec & -0.01 & 0.01 & 0.01 & -0.16 & -0.19 & 0.04 & 0.03 & -0.02 & 0.39 & -0.02 \\
\hline UVB & 0.00 & 0.00 & 0.00 & 0.02 & 0.03 & -0.01 & 0.00 & 0.02 & -0.02 & 0.02 \\
\hline Correl. Coeff. & $\begin{array}{c}\text { Subst. } \\
\text { rate }\end{array}$ & Diversif & Body size & Elev & Lat & Temp & HR & NPP & Prec & UVB \\
\hline Subst. rate & 1.00 & 0.31 & -0.19 & -0.38 & -0.09 & 0.56 & 0.00 & 0.14 & -0.03 & 0.01 \\
\hline Diversif & 0.31 & 1.00 & -0.11 & -0.04 & -0.10 & 0.09 & 0.01 & -0.04 & 0.03 & 0.06 \\
\hline Body size & -0.19 & -0.11 & 1.00 & 0.11 & 0.04 & -0.12 & 0.14 & 0.01 & 0.09 & 0.04 \\
\hline Elev & -0.38 & -0.04 & 0.11 & 1.00 & 0.10 & -0.55 & -0.26 & -0.15 & -0.22 & 0.10 \\
\hline Lat & -0.09 & -0.10 & 0.04 & 0.10 & 1.00 & -0.11 & 0.01 & 0.21 & -0.15 & 0.10 \\
\hline Temp & 0.56 & 0.09 & -0.12 & -0.55 & -0.11 & 1.00 & 0.17 & 0.26 & 0.32 & -0.24 \\
\hline $\mathrm{HR}$ & 0.00 & 0.01 & 0.14 & -0.26 & 0.01 & 0.17 & 1.00 & -0.14 & 0.53 & -0.24 \\
\hline NPP & 0.14 & -0.04 & 0.01 & -0.15 & 0.21 & 0.26 & -0.14 & 1.00 & -0.05 & 0.23 \\
\hline Prec & -0.03 & 0.03 & 0.09 & -0.22 & -0.15 & 0.32 & 0.53 & -0.05 & 1.00 & -0.27 \\
\hline UVB & 0.01 & 0.06 & 0.04 & 0.10 & 0.10 & -0.24 & -0.24 & 0.23 & -0.27 & 1.00 \\
\hline Post. Prob. & $\begin{array}{c}\text { Subst. } \\
\text { rate }\end{array}$ & Diversif & Body size & Elev & Lat & Temp & HR & NPP & Prec & UVB \\
\hline Subst. rate & - & 1.00 & 0.08 & 0.00 & 0.28 & 1.00 & 0.49 & 0.82 & 0.41 & 0.53 \\
\hline Diversif & 1.00 & - & 0.14 & 0.34 & 0.16 & 0.81 & 0.53 & 0.35 & 0.62 & 0.74 \\
\hline Body size & 0.08 & 0.14 & - & 0.87 & 0.66 & 0.12 & 0.91 & 0.52 & 0.81 & 0.66 \\
\hline Elev & 0.00 & 0.34 & 0.87 & - & 0.84 & 0.00 & 0.00 & 0.08 & 0.02 & 0.84 \\
\hline Lat & 0.28 & 0.16 & 0.66 & 0.84 & - & 0.14 & 0.54 & 0.98 & 0.06 & 0.84 \\
\hline Temp & 1.00 & 0.81 & 0.12 & 0.00 & 0.14 & - & 0.96 & 0.99 & 1.00 & 0.01 \\
\hline$H R$ & 0.49 & 0.53 & 0.91 & 0.00 & 0.54 & 0.96 & - & 0.08 & 1.00 & 0.01 \\
\hline NPP & 0.82 & 0.35 & 0.52 & 0.08 & 0.98 & 0.99 & 0.08 & - & 0.29 & 0.99 \\
\hline Prec & 0.41 & 0.62 & 0.81 & 0.02 & 0.06 & 1.00 & 1.00 & 0.29 & - & 0.00 \\
\hline UVB & 0.53 & 0.74 & 0.66 & 0.84 & 0.84 & 0.01 & 0.01 & 0.99 & 0.00 & - \\
\hline
\end{tabular}




\begin{tabular}{|c|c|c|c|c|c|c|c|c|c|c|}
\hline Precisions & $\begin{array}{c}\text { Subst. } \\
\text { rate }\end{array}$ & Diversif & Body size & Elev & Lat & Temp & HR & NPP & Prec & UVB \\
\hline Subst. rate & 5.84 & -3.22 & 1.18 & 0.32 & 0.04 & 12.00 & -0.73 & 0.25 & 1.41 & -2.72 \\
\hline Diversif & -3.22 & 16.00 & 1.38 & $\begin{array}{c}- \\
0.31\end{array}$ & 0.15 & 3.63 & -0.71 & 0.36 & -0.83 & -2.49 \\
\hline Body size & 1.18 & 1.38 & 26.70 & $\begin{array}{c}- \\
0.48\end{array}$ & 0.00 & 0.86 & -9.48 & $\begin{array}{c}- \\
0.46\end{array}$ & -0.50 & -3.11 \\
\hline Elev & 0.32 & -0.31 & -0.48 & 1.22 & $\begin{array}{c}- \\
0.05\end{array}$ & 3.43 & 3.44 & 0.10 & -0.07 & 0.51 \\
\hline Lat & 0.04 & 0.15 & 0.00 & $\begin{array}{c}- \\
0.05\end{array}$ & 0.31 & 0.23 & -1.17 & $\begin{array}{c}- \\
0.25\end{array}$ & 0.20 & -0.06 \\
\hline Temp & 12.00 & 3.63 & 0.86 & 3.43 & 0.23 & 84.90 & 8.54 & $\begin{array}{c}- \\
5.05\end{array}$ & -6.81 & 22.10 \\
\hline $\mathrm{HR}$ & -0.73 & -0.71 & -9.48 & 3.44 & $\begin{array}{c}- \\
1.17\end{array}$ & 8.54 & 181.00 & 3.74 & $\begin{array}{c}- \\
14.20\end{array}$ & 12.10 \\
\hline NPP & 0.25 & 0.36 & -0.46 & 0.10 & $\begin{array}{c}- \\
0.25\end{array}$ & -5.05 & 3.74 & 3.50 & 0.05 & -4.42 \\
\hline Prec & 1.41 & -0.83 & -0.50 & $\begin{array}{c}- \\
0.07\end{array}$ & 0.20 & -6.81 & -14.20 & 0.05 & 5.05 & 1.34 \\
\hline UVB & -2.72 & -2.49 & -3.11 & 0.51 & $\begin{array}{c}- \\
0.06\end{array}$ & 22.10 & 12.10 & $\begin{array}{c}- \\
4.42\end{array}$ & 1.34 & 76.40 \\
\hline Partial C.C. & $\begin{array}{c}\text { Subst. } \\
\text { rate }\end{array}$ & Diversif & Body size & Elev & Lat & Temp & HR & NPP & Prec & UVB \\
\hline Subst. rate & -1.00 & 0.33 & -0.09 & $\begin{array}{c}- \\
0.12\end{array}$ & $\begin{array}{c}- \\
0.03\end{array}$ & 0.53 & 0.02 & $\begin{array}{c}- \\
0.06\end{array}$ & -0.25 & 0.13 \\
\hline Diversif & 0.33 & -1.00 & -0.07 & 0.07 & $\begin{array}{c}- \\
0.07\end{array}$ & -0.09 & 0.01 & $\begin{array}{c}- \\
0.05\end{array}$ & 0.09 & 0.07 \\
\hline Body size & -0.09 & -0.07 & -1.00 & 0.08 & 0.00 & -0.02 & 0.14 & 0.05 & 0.04 & 0.07 \\
\hline Elev & -0.12 & 0.07 & 0.08 & 1.00 & 0.08 & -0.34 & -0.23 & 0.05 & 0.03 & -0.05 \\
\hline Lat & -0.03 & -0.07 & 0.00 & 0.08 & 1.00 & -0.05 & 0.15 & 0.23 & -0.15 & 0.01 \\
\hline Temp & 0.53 & -0.09 & -0.02 & $\begin{array}{c}- \\
0.34\end{array}$ & $\begin{array}{c}- \\
0.05\end{array}$ & -1.00 & -0.07 & 0.29 & 0.32 & -0.27 \\
\hline $\mathrm{HR}$ & 0.02 & 0.01 & 0.14 & $\begin{array}{c}- \\
0.23\end{array}$ & 0.15 & -0.07 & -1.00 & $\begin{array}{c}- \\
0.15\end{array}$ & 0.47 & -0.10 \\
\hline NPP & -0.06 & -0.05 & 0.05 & $\begin{array}{c}- \\
0.05\end{array}$ & 0.23 & 0.29 & -0.15 & $\begin{array}{c}- \\
1.00\end{array}$ & -0.01 & 0.27 \\
\hline Prec & -0.25 & 0.09 & 0.04 & 0.03 & $\begin{array}{c}- \\
0.15\end{array}$ & 0.32 & 0.47 & $\begin{array}{c}- \\
0.01\end{array}$ & -1.00 & -0.07 \\
\hline UVB & 0.13 & 0.07 & 0.07 & $\begin{array}{c}- \\
0.05 \\
\end{array}$ & 0.01 & -0.27 & -0.10 & 0.27 & -0.07 & -1.00 \\
\hline Post. Probab. & $\begin{array}{c}\text { Subst. } \\
\text { rate }\end{array}$ & Diversif & Body size & Elev & Lat & Temp & HR & NPP & Prec & UVB \\
\hline Subst. rate & - & 0.99 & 0.28 & 0.23 & 0.44 & 1.00 & 0.55 & 0.38 & 0.07 & 0.79 \\
\hline Diversif & 0.99 & - & 0.26 & 0.74 & 0.27 & 0.22 & 0.55 & 0.33 & 0.80 & 0.76 \\
\hline Body size & 0.28 & 0.26 & - & 0.80 & 0.5 & 0.42 & 0.91 & 0.68 & 0.67 & 0.76 \\
\hline Elev & 0.23 & 0.74 & 0.80 & - & 0.79 & 0.00 & 0.01 & 0.31 & 0.6 & 0.29 \\
\hline Lat & 0.44 & 0.27 & 0.5 & 0.79 & - & 0.36 & 0.94 & 0.99 & 0.07 & 0.55 \\
\hline Temp & 1.00 & 0.22 & 0.42 & 0.00 & 0.36 & - & 0.29 & 0.99 & 1.00 & 0.01 \\
\hline $\mathrm{HR}$ & 0.55 & 0.55 & 0.91 & 0.01 & 0.94 & 0.29 & - & 0.07 & 1.00 & 0.15 \\
\hline NPP & 0.38 & 0.33 & 0.68 & 0.31 & 0.99 & 0.99 & 0.07 & - & 0.47 & 1.00 \\
\hline Prec & 0.07 & 0.80 & 0.67 & 0.60 & 0.07 & 1.00 & 1.00 & 0.47 & - & 0.25 \\
\hline UVB & 0.79 & 0.76 & 0.76 & 0.29 & 0.55 & 0.01 & 0.15 & 1.00 & 0.25 & - \\
\hline
\end{tabular}




\begin{tabular}{|c|c|c|c|c|c|c|c|c|c|}
\hline \multicolumn{10}{|c|}{ MITOCHONDRIAL NON-CODING GENES: ANALYSIS 1 (Controlling for Temperature) } \\
\hline Covariances & $\begin{array}{c}\text { Subst. } \\
\text { rate }\end{array}$ & Diversif & Body Size & Elev & Lat & HR & NPP & Prec & UVB \\
\hline Subst. rate & 0.30 & 0.05 & -0.02 & -0.06 & -0.03 & -0.01 & 0.00 & -0.09 & 0.01 \\
\hline Diversif & 0.05 & 0.08 & -0.01 & 0.00 & -0.05 & 0.00 & -0.01 & 0.00 & 0.00 \\
\hline Body Size & -0.02 & -0.01 & 0.05 & 0.01 & 0.01 & 0.00 & 0.01 & 0.02 & 0.00 \\
\hline Elev & -0.06 & 0.00 & 0.01 & 1.01 & 0.10 & -0.02 & 0.00 & -0.03 & 0.00 \\
\hline Lat & -0.03 & -0.05 & 0.01 & 0.10 & 4.03 & 0.01 & 0.32 & -0.14 & 0.02 \\
\hline$H R$ & -0.01 & 0.00 & 0.00 & -0.02 & 0.01 & 0.01 & -0.01 & 0.03 & 0.00 \\
\hline NPP & 0.00 & -0.01 & 0.01 & 0.00 & 0.32 & -0.01 & 0.40 & -0.06 & 0.03 \\
\hline Prec & -0.09 & 0.00 & 0.02 & -0.03 & -0.14 & 0.03 & -0.06 & 0.34 & -0.02 \\
\hline UVB & 0.01 & 0.00 & 0.00 & 0.00 & 0.02 & 0.00 & 0.03 & -0.02 & 0.02 \\
\hline Correl. Coeff. & $\begin{array}{c}\text { Subst. } \\
\text { rate }\end{array}$ & Diversif & Body Size & Elev & Lat & HR & NPP & Prec & UVB \\
\hline Subst. rate & 1.00 & 0.32 & -0.15 & -0.10 & -0.03 & -0.12 & -0.01 & -0.27 & 0.18 \\
\hline Diversif & 0.32 & 1.00 & -0.10 & 0.01 & -0.09 & -0.01 & -0.07 & 0.00 & 0.09 \\
\hline Body Size & -0.15 & -0.10 & 1.00 & 0.05 & 0.03 & 0.16 & 0.04 & 0.14 & 0.02 \\
\hline Elev & -0.10 & 0.01 & 0.05 & 1.00 & 0.05 & -0.20 & 0.00 & -0.05 & -0.03 \\
\hline Lat & -0.03 & -0.09 & 0.03 & 0.05 & 1.00 & 0.03 & 0.25 & -0.12 & 0.08 \\
\hline $\mathrm{HR}$ & -0.12 & -0.01 & 0.16 & -0.20 & 0.03 & 1.00 & -0.20 & 0.51 & -0.21 \\
\hline NPP & -0.01 & -0.07 & 0.04 & 0.00 & 0.25 & -0.20 & 1.00 & -0.15 & 0.31 \\
\hline Prec & -0.27 & 0.00 & 0.14 & -0.05 & -0.12 & 0.51 & -0.15 & 1.00 & -0.21 \\
\hline UVB & 0.18 & 0.09 & 0.02 & -0.03 & 0.08 & -0.21 & 0.31 & -0.21 & 1.00 \\
\hline Post. Prob. & $\begin{array}{c}\text { Subst. } \\
\text { rate }\end{array}$ & Diversif & Body Size & Elev & Lat & $H R$ & NPP & Prec & UVB \\
\hline Subst. rate & - & 1.00 & 0.15 & 0.24 & 0.42 & 0.22 & 0.48 & 0.05 & 0.88 \\
\hline Diversif & 1.00 & - & 0.16 & 0.54 & 0.18 & 0.48 & 0.26 & 0.50 & 0.82 \\
\hline Body Size & 0.15 & 0.16 & - & 0.71 & 0.61 & 0.94 & 0.65 & 0.92 & 0.56 \\
\hline Elev & 0.24 & 0.54 & 0.71 & - & 0.69 & 0.02 & 0.50 & 0.32 & 0.38 \\
\hline Lat & 0.42 & 0.18 & 0.61 & 0.69 & - & 0.61 & 0.99 & 0.12 & 0.78 \\
\hline$H R$ & 0.22 & 0.48 & 0.94 & 0.02 & 0.61 & - & 0.03 & 1.00 & 0.02 \\
\hline NPP & 0.48 & 0.26 & 0.65 & 0.50 & 0.99 & 0.03 & - & 0.06 & 1.00 \\
\hline Prec & 0.05 & 0.50 & 0.92 & 0.32 & 0.12 & 1.00 & 0.06 & - & 0.02 \\
\hline UVB & 0.88 & 0.82 & 0.56 & 0.38 & 0.78 & 0.02 & 1.00 & 0.02 & - \\
\hline
\end{tabular}




\begin{tabular}{|c|c|c|c|c|c|c|c|c|c|c|}
\hline \multicolumn{11}{|c|}{ MITOHONDRIAL NON-CODING GENES: ANALYSIS 2} \\
\hline Covariances & $\begin{array}{c}\text { Subst. } \\
\text { rate }\end{array}$ & Diversif & Body size & Elev & Lat & Temp & HR & NPP & Prec & UVB \\
\hline Subst. rate & 0.45 & 0.06 & -0.03 & -0.31 & -0.12 & 0.07 & 0.00 & 0.06 & -0.01 & 0.00 \\
\hline Diversif & 0.06 & 0.08 & -0.01 & -0.02 & -0.06 & 0.00 & 0.00 & -0.01 & 0.01 & 0.00 \\
\hline Body size & -0.03 & -0.01 & 0.05 & 0.03 & 0.02 & 0.00 & 0.00 & 0.00 & 0.01 & 0.00 \\
\hline Elev & -0.31 & -0.02 & 0.03 & 1.47 & 0.26 & -0.12 & -0.03 & -0.12 & -0.16 & 0.02 \\
\hline Lat & -0.12 & -0.06 & 0.02 & 0.26 & 4.15 & -0.04 & 0.00 & 0.28 & -0.19 & 0.03 \\
\hline Temp & 0.07 & 0.00 & 0.00 & -0.12 & -0.04 & 0.03 & 0.00 & 0.03 & 0.04 & -0.01 \\
\hline $\mathrm{HR}$ & 0.00 & 0.00 & 0.00 & -0.03 & 0.00 & 0.00 & 0.01 & -0.01 & 0.03 & 0.00 \\
\hline NPP & 0.06 & -0.01 & 0.00 & -0.12 & 0.28 & 0.03 & -0.01 & 0.43 & -0.02 & 0.02 \\
\hline Prec & -0.01 & 0.01 & 0.01 & -0.16 & -0.19 & 0.04 & 0.03 & -0.02 & 0.39 & -0.02 \\
\hline UVB & 0.00 & 0.00 & 0.00 & 0.02 & 0.03 & -0.01 & 0.00 & 0.02 & -0.02 & 0.02 \\
\hline Correl. Coeff. & $\begin{array}{c}\text { Subst. } \\
\text { rate }\end{array}$ & Diversif & Body size & Elev & Lat & Temp & HR & NPP & Prec & UVB \\
\hline Subst. rate & 1.00 & 0.31 & -0.19 & -0.38 & -0.09 & 0.56 & 0.00 & 0.14 & -0.03 & 0.00 \\
\hline Diversif & 0.31 & 1.00 & -0.11 & -0.04 & -0.10 & 0.09 & 0.01 & -0.04 & 0.03 & 0.06 \\
\hline Body size & -0.19 & -0.11 & 1.00 & 0.12 & 0.04 & -0.12 & 0.14 & 0.01 & 0.09 & 0.04 \\
\hline Elev & -0.38 & -0.04 & 0.12 & 1.00 & 0.11 & -0.55 & -0.26 & -0.15 & -0.22 & 0.11 \\
\hline Lat & -0.09 & -0.10 & 0.04 & 0.11 & 1.00 & -0.12 & 0.01 & 0.21 & -0.15 & 0.10 \\
\hline Temp & 0.56 & 0.09 & -0.12 & -0.55 & -0.12 & 1.00 & 0.17 & 0.26 & 0.32 & -0.24 \\
\hline $\mathrm{HR}$ & 0.00 & 0.01 & 0.14 & -0.26 & 0.01 & 0.17 & 1.00 & -0.14 & 0.53 & -0.24 \\
\hline NPP & 0.14 & -0.04 & 0.01 & -0.15 & 0.21 & 0.26 & -0.14 & 1.00 & -0.05 & 0.23 \\
\hline Prec & -0.03 & 0.03 & 0.09 & -0.22 & -0.15 & 0.32 & 0.53 & -0.05 & 1.00 & -0.27 \\
\hline UVB & 0.00 & 0.06 & 0.04 & 0.11 & 0.10 & -0.24 & -0.24 & 0.23 & -0.27 & 1.00 \\
\hline Post. Prob. & $\begin{array}{c}\text { Subst. } \\
\text { rate }\end{array}$ & Diversif & Body size & Elev & Lat & Temp & HR & NPP & Prec & UVB \\
\hline Subst. rate & - & 1.00 & 0.07 & 0.00 & 0.27 & 1.00 & 0.49 & 0.81 & 0.41 & 0.52 \\
\hline Diversif & 1.00 & - & 0.13 & 0.33 & 0.15 & 0.81 & 0.55 & 0.33 & 0.62 & 0.73 \\
\hline Body size & 0.07 & 0.13 & - & 0.87 & 0.67 & 0.12 & 0.90 & 0.52 & 0.81 & 0.67 \\
\hline Elev & 0.00 & 0.33 & 0.87 & - & 0.85 & 0.00 & 0.01 & 0.07 & 0.02 & 0.85 \\
\hline Lat & 0.27 & 0.15 & 0.67 & 0.85 & - & 0.13 & 0.52 & 0.98 & 0.06 & 0.84 \\
\hline Temp & 1.00 & 0.81 & 0.12 & 0.00 & 0.13 & - & 0.95 & 0.99 & 1.00 & 0.01 \\
\hline $\mathrm{HR}$ & 0.49 & 0.55 & 0.90 & 0.01 & 0.52 & 0.95 & - & 0.09 & 1.00 & 0.01 \\
\hline NPP & 0.81 & 0.33 & 0.52 & 0.07 & 0.98 & 0.99 & 0.09 & - & 0.30 & 0.99 \\
\hline Prec & 0.41 & 0.62 & 0.81 & 0.02 & 0.06 & 1.00 & 1.00 & 0.30 & - & 0.00 \\
\hline UVB & 0.52 & 0.73 & 0.67 & 0.85 & 0.84 & 0.01 & 0.01 & 0.99 & 0.00 & \\
\hline
\end{tabular}




\begin{tabular}{|c|c|c|c|c|c|c|c|c|c|c|}
\hline Precisions & $\begin{array}{l}\text { Subst. } \\
\text { rate }\end{array}$ & Diversif & Body size & Elev & Lat & Temp & HR & NPP & Prec & UVB \\
\hline Subst. rate & 5.83 & -3.18 & 1.18 & 0.33 & 0.04 & -11.90 & -0.70 & 0.26 & 1.41 & -2.65 \\
\hline Diversif & -3.18 & 15.90 & 1.38 & -0.30 & 0.15 & 3.54 & -0.76 & 0.39 & -0.83 & -2.49 \\
\hline Body size & 1.18 & 1.38 & 26.70 & -0.48 & -0.01 & 0.87 & -9.31 & -0.45 & -0.52 & -3.00 \\
\hline Elev & 0.33 & -0.30 & -0.48 & 1.22 & -0.05 & 3.41 & 3.46 & 0.11 & -0.07 & 0.49 \\
\hline Lat & 0.04 & 0.15 & -0.01 & -0.05 & 0.31 & 0.25 & -1.16 & -0.25 & 0.19 & -0.05 \\
\hline Temp & -11.90 & 3.54 & 0.87 & 3.41 & 0.25 & 85.00 & 8.61 & -5.12 & -6.83 & 22.10 \\
\hline $\mathrm{HR}$ & -0.70 & -0.76 & -9.31 & 3.46 & -1.16 & 8.61 & 181.00 & 3.70 & -14.20 & 12.10 \\
\hline NPP & 0.26 & 0.39 & -0.45 & 0.11 & -0.25 & -5.12 & 3.70 & 3.51 & 0.05 & -4.45 \\
\hline Prec & 1.41 & -0.83 & -0.52 & -0.07 & 0.19 & -6.83 & -14.20 & 0.05 & 5.03 & 1.30 \\
\hline UVB & -2.65 & -2.49 & -3.00 & 0.49 & -0.05 & 22.10 & 12.10 & -4.45 & 1.30 & 76.30 \\
\hline Partial C.C. & $\begin{array}{c}\text { Subst. } \\
\text { rate }\end{array}$ & Diversif & Body size & Elev & Lat & Temp & HR & NPP & Prec & UVB \\
\hline Subst. rate & -1.00 & 0.33 & -0.09 & -0.12 & -0.03 & 0.52 & 0.02 & -0.06 & -0.26 & 0.12 \\
\hline Diversif & 0.33 & -1.00 & -0.07 & 0.07 & -0.07 & -0.09 & 0.01 & -0.05 & 0.09 & 0.07 \\
\hline Body size & -0.09 & -0.07 & -1.00 & 0.08 & 0.00 & -0.02 & 0.13 & 0.05 & 0.05 & 0.07 \\
\hline Elev & -0.12 & 0.07 & 0.08 & -1.00 & 0.08 & -0.34 & -0.23 & -0.05 & 0.03 & -0.05 \\
\hline Lat & -0.03 & -0.07 & 0.00 & 0.08 & -1.00 & -0.05 & 0.15 & 0.23 & -0.15 & 0.01 \\
\hline Temp & 0.52 & -0.09 & -0.02 & -0.34 & -0.05 & -1.00 & -0.07 & 0.29 & 0.33 & -0.27 \\
\hline$H R$ & 0.02 & 0.01 & 0.13 & -0.23 & 0.15 & -0.07 & -1.00 & -0.15 & 0.47 & -0.10 \\
\hline NPP & -0.06 & -0.05 & 0.05 & -0.05 & 0.23 & 0.29 & -0.15 & -1.00 & -0.01 & 0.27 \\
\hline Prec & -0.26 & 0.09 & 0.05 & 0.03 & -0.15 & 0.33 & 0.47 & -0.01 & -1.00 & -0.07 \\
\hline UVB & 0.12 & 0.07 & 0.07 & -0.05 & 0.01 & -0.27 & -0.10 & 0.27 & -0.07 & -1.00 \\
\hline Post. Probab. & $\begin{array}{c}\text { Subst. } \\
\text { rate }\end{array}$ & Diversif & Body size & Elev & Lat & Temp & HR & NPP & Prec & UVB \\
\hline Subst. rate & - & 1 & 0.27 & 0.22 & 0.43 & 1.00 & 0.54 & 0.37 & 0.06 & 0.79 \\
\hline Diversif & 1 & - & 0.26 & 0.73 & 0.28 & 0.22 & 0.56 & 0.32 & 0.80 & 0.75 \\
\hline Body size & 0.27 & 0.26 & - & 0.80 & 0.51 & 0.43 & 0.91 & 0.68 & 0.68 & 0.74 \\
\hline Elev & 0.22 & 0.73 & 0.80 & - & 0.78 & 0.01 & 0.01 & 0.31 & 0.61 & 0.31 \\
\hline Lat & 0.43 & 0.28 & 0.51 & 0.78 & - & 0.35 & 0.94 & 0.99 & 0.068 & 0.54 \\
\hline Temp & 1.00 & 0.22 & 0.43 & 0.01 & 0.35 & - & 0.28 & 0.99 & 1.00 & 0.01 \\
\hline$H R$ & 0.54 & 0.56 & 0.91 & 0.01 & 0.94 & 0.28 & - & 0.07 & 1.00 & 0.15 \\
\hline NPP & 0.37 & 0.32 & 0.68 & 0.31 & 0.99 & 0.99 & 0.07 & - & 0.46 & 1.00 \\
\hline Prec & 0.06 & 0.80 & 0.68 & 0.61 & 0.07 & 1.00 & 1.00 & 0.46 & - & 0.26 \\
\hline UVB & 0.79 & 0.75 & 0.74 & 0.31 & 0.54 & 0.01 & 0.15 & 1.00 & 0.26 & - \\
\hline
\end{tabular}




\begin{tabular}{|c|c|c|c|c|c|c|c|c|c|}
\hline \multicolumn{10}{|c|}{ MITOCHONDRIAL NON-CODING GENES: ANALYSIS 2 (Controlling for Temperature) } \\
\hline Covariances & $\begin{array}{l}\text { Subst. } \\
\text { rate }\end{array}$ & Diversif & Body Size & Elev & Lat & HR & NPP & Prec & UVB \\
\hline Subst. rate & 0.30 & 0.05 & -0.02 & -0.06 & -0.04 & -0.01 & -0.01 & -0.09 & 0.01 \\
\hline Diversif & 0.05 & 0.08 & -0.01 & 0.00 & -0.05 & 0.00 & -0.01 & 0.00 & 0.00 \\
\hline Body Size & -0.02 & -0.01 & 0.05 & 0.01 & 0.01 & 0.00 & 0.01 & 0.02 & 0.00 \\
\hline Elev & -0.06 & 0.00 & 0.01 & 1.01 & 0.10 & -0.02 & 0.00 & -0.03 & 0.00 \\
\hline Lat & -0.04 & -0.05 & 0.01 & 0.10 & 4.04 & 0.01 & 0.32 & -0.14 & 0.02 \\
\hline $\mathrm{HR}$ & -0.01 & 0.00 & 0.00 & -0.02 & 0.01 & 0.01 & -0.01 & 0.03 & 0.00 \\
\hline NPP & -0.01 & -0.01 & 0.01 & 0.00 & 0.32 & -0.01 & 0.40 & -0.06 & 0.03 \\
\hline Prec & -0.09 & 0.00 & 0.02 & -0.03 & -0.14 & 0.03 & -0.06 & 0.35 & -0.02 \\
\hline UVB & 0.01 & 0.00 & 0.00 & 0.00 & 0.02 & 0.00 & 0.03 & -0.02 & 0.02 \\
\hline Correl. Coeff. & $\begin{array}{c}\text { Subst. } \\
\text { rate }\end{array}$ & Diversif & Body Size & Elev & Lat & HR & NPP & Prec & UVB \\
\hline Subst. rate & 1.00 & 0.32 & -0.15 & -0.11 & -0.03 & -0.12 & -0.01 & -0.27 & 0.17 \\
\hline Diversif & 0.32 & 1.00 & -0.10 & 0.01 & -0.09 & -0.01 & -0.07 & 0.00 & 0.09 \\
\hline Body Size & -0.15 & -0.10 & 1.00 & 0.06 & 0.03 & 0.16 & 0.04 & 0.14 & 0.02 \\
\hline Elev & -0.11 & 0.01 & 0.06 & 1.00 & 0.05 & -0.20 & 0.00 & -0.05 & -0.03 \\
\hline Lat & -0.03 & -0.09 & 0.03 & 0.05 & 1.00 & 0.03 & 0.25 & -0.12 & 0.07 \\
\hline $\mathrm{HR}$ & -0.12 & -0.01 & 0.16 & -0.20 & 0.03 & 1.00 & -0.19 & 0.51 & -0.21 \\
\hline NPP & -0.01 & -0.07 & 0.04 & 0.00 & 0.25 & -0.19 & 1.00 & -0.15 & 0.31 \\
\hline Prec & -0.27 & 0.00 & 0.14 & -0.05 & -0.12 & 0.51 & -0.15 & 1.00 & -0.20 \\
\hline UVB & 0.17 & 0.09 & 0.02 & -0.03 & 0.07 & -0.21 & 0.31 & -0.20 & 1.00 \\
\hline Post. Prob. & $\begin{array}{c}\text { Subst. } \\
\text { rate }\end{array}$ & Diversif & Body Size & Elev & Lat & HR & NPP & Prec & UVB \\
\hline Subst. rate & - & 1.00 & 0.15 & 0.24 & 0.42 & 0.23 & 0.47 & 0.05 & 0.87 \\
\hline Diversif & 1.00 & - & 0.15 & 0.54 & 0.18 & 0.48 & 0.24 & 0.50 & 0.81 \\
\hline Body Size & 0.15 & 0.15 & - & 0.71 & 0.62 & 0.94 & 0.66 & 0.91 & 0.56 \\
\hline Elev & 0.24 & 0.54 & 0.71 & - & 0.69 & 0.02 & 0.49 & 0.33 & 0.38 \\
\hline Lat & 0.42 & 0.18 & 0.62 & 0.69 & - & 0.60 & 0.99 & 0.12 & 0.77 \\
\hline $\mathrm{HR}$ & 0.23 & 0.48 & 0.94 & 0.02 & 0.60 & - & 0.02 & 1.00 & 0.02 \\
\hline NPP & 0.47 & 0.24 & 0.66 & 0.49 & 0.99 & 0.02 & - & 0.07 & 1.00 \\
\hline Prec & 0.05 & 0.50 & 0.91 & 0.33 & 0.12 & 1.00 & 0.07 & - & 0.02 \\
\hline UVB & 0.87 & 0.81 & 0.56 & 0.38 & 0.77 & 0.02 & 1.00 & 0.02 & - \\
\hline
\end{tabular}




\begin{tabular}{|c|c|c|c|c|c|c|c|c|c|c|c|}
\hline \multicolumn{12}{|c|}{ MITOCHONDRIAL CODING-GENE: dN ANALYSIS 1} \\
\hline Covariances & dS & $d \mathbf{N}$ & Diversif & Body Size & Elev & Lat & Temp & HR & NPP & Prec & UVB \\
\hline dS & 0.25 & 0.14 & 0.03 & -0.02 & -0.16 & -0.11 & 0.05 & 0.00 & -0.03 & -0.01 & -0.02 \\
\hline$d N$ & 0.14 & 0.34 & 0.03 & 0.00 & -0.20 & 0.11 & 0.05 & 0.01 & 0.04 & 0.04 & -0.01 \\
\hline Diversif & 0.03 & 0.03 & 0.09 & -0.01 & -0.02 & -0.06 & 0.01 & 0.00 & -0.01 & 0.01 & 0.00 \\
\hline Body Size & -0.02 & 0.00 & -0.01 & 0.04 & 0.03 & 0.03 & 0.00 & 0.00 & 0.01 & 0.01 & 0.00 \\
\hline Elev & -0.16 & -0.20 & -0.02 & 0.03 & 1.35 & 0.27 & -0.11 & -0.03 & -0.11 & -0.18 & 0.02 \\
\hline Lat & -0.11 & 0.11 & -0.06 & 0.03 & 0.27 & 3.88 & -0.05 & 0.00 & 0.24 & -0.22 & 0.04 \\
\hline Temp & 0.05 & 0.05 & 0.01 & 0.00 & -0.11 & -0.05 & 0.03 & 0.00 & 0.03 & 0.05 & -0.01 \\
\hline $\mathrm{HR}$ & 0.00 & 0.01 & 0.00 & 0.00 & -0.03 & 0.00 & 0.00 & 0.01 & -0.01 & 0.03 & 0.00 \\
\hline NPP & -0.03 & 0.04 & -0.01 & 0.01 & -0.11 & 0.24 & 0.03 & -0.01 & 0.41 & -0.02 & 0.02 \\
\hline Prec & -0.01 & 0.04 & 0.01 & 0.01 & -0.18 & -0.22 & 0.05 & 0.03 & -0.02 & 0.38 & -0.02 \\
\hline UVB & -0.02 & -0.01 & 0.00 & 0.00 & 0.02 & 0.04 & -0.01 & 0.00 & 0.02 & -0.02 & 0.02 \\
\hline Correl. Coeff. & dS & $d N$ & Diversif & Body Size & Elev & Lat & Temp & HR & NPP & Prec & UVB \\
\hline dS & 1.00 & 0.50 & 0.24 & -0.21 & -0.28 & -0.11 & 0.58 & 0.10 & -0.08 & -0.01 & -0.26 \\
\hline $\mathrm{dN}$ & 0.50 & 1.00 & 0.16 & 0.00 & -0.32 & 0.09 & 0.50 & 0.19 & 0.11 & 0.12 & -0.16 \\
\hline Diversif & 0.24 & 0.16 & 1.00 & -0.11 & -0.05 & -0.11 & 0.11 & 0.02 & -0.03 & 0.06 & 0.04 \\
\hline Body Size & -0.21 & 0.00 & -0.11 & 1.00 & 0.11 & 0.08 & -0.09 & 0.09 & 0.04 & 0.06 & 0.07 \\
\hline Elev & -0.28 & -0.32 & -0.05 & 0.11 & 1.00 & 0.12 & -0.55 & -0.30 & -0.14 & -0.25 & 0.12 \\
\hline Lat & -0.11 & 0.09 & -0.11 & 0.08 & 0.12 & 1.00 & -0.14 & 0.01 & 0.19 & -0.18 & 0.14 \\
\hline Temp & 0.58 & 0.50 & 0.11 & -0.09 & -0.55 & -0.14 & 1.00 & 0.27 & 0.22 & 0.42 & -0.32 \\
\hline $\mathrm{HR}$ & 0.10 & 0.19 & 0.02 & 0.09 & -0.30 & 0.01 & 0.27 & 1.00 & -0.11 & 0.53 & -0.25 \\
\hline NPP & -0.08 & 0.11 & -0.03 & 0.04 & -0.14 & 0.19 & 0.22 & -0.11 & 1.00 & -0.04 & 0.21 \\
\hline Prec & -0.01 & 0.12 & 0.06 & 0.06 & -0.25 & -0.18 & 0.42 & 0.53 & -0.04 & 1.00 & -0.28 \\
\hline UVB & -0.26 & -0.16 & 0.04 & 0.07 & 0.12 & 0.14 & -0.32 & -0.25 & 0.21 & -0.28 & 1.00 \\
\hline Post. Prob. & dS & $\mathrm{dN}$ & Diversif & Body Size & Elev & Lat & Temp & HR & NPP & Prec & UVB \\
\hline dS & - & 0.98 & 0.97 & 0.06 & 0.03 & 0.25 & 1.00 & 0.73 & 0.32 & 0.46 & 0.04 \\
\hline $\mathrm{dN}$ & 0.98 & - & 0.86 & 0.51 & 0.05 & 0.65 & 0.99 & 0.81 & 0.69 & 0.70 & 0.22 \\
\hline Diversif & 0.97 & 0.86 & - & 0.15 & 0.30 & 0.14 & 0.86 & 0.58 & 0.38 & 0.71 & 0.64 \\
\hline Body Size & 0.06 & 0.51 & 0.15 & - & 0.85 & 0.77 & 0.19 & 0.80 & 0.66 & 0.70 & 0.73 \\
\hline Elev & 0.03 & 0.05 & 0.30 & 0.85 & - & 0.87 & 0.00 & 0.00 & 0.09 & 0.01 & 0.88 \\
\hline Lat & 0.25 & 0.65 & 0.14 & 0.77 & 0.87 & - & 0.09 & 0.52 & 0.97 & 0.04 & 0.91 \\
\hline Temp & 1.00 & 0.99 & 0.86 & 0.19 & 0.00 & 0.09 & - & 0.99 & 0.98 & 1.00 & 0.00 \\
\hline $\mathrm{HR}$ & 0.73 & 0.81 & 0.58 & 0.80 & 0.00 & 0.52 & 0.99 & - & 0.16 & 1.00 & 0.01 \\
\hline NPP & 0.32 & 0.69 & 0.38 & 0.66 & 0.09 & 0.97 & 0.98 & 0.16 & - & 0.35 & 0.98 \\
\hline Prec & 0.46 & 0.70 & 0.71 & 0.70 & 0.01 & 0.04 & 1.00 & 1.00 & 0.35 & - & 0.00 \\
\hline UVB & 0.04 & 0.22 & 0.64 & 0.73 & 0.88 & 0.91 & 0.00 & 0.01 & 0.98 & 0.00 & - \\
\hline
\end{tabular}




\begin{tabular}{|c|c|c|c|c|c|c|c|c|c|c|c|}
\hline Precisions & dS & $d N$ & Diversif & Body Size & Elev & Lat & Temp & HR & NPP & Prec & UVB \\
\hline dS & 18.30 & -5.81 & -3.44 & 5.02 & -0.46 & 0.16 & -27.00 & -4.56 & 2.35 & 4.47 & 4.23 \\
\hline $\mathrm{dN}$ & -5.81 & 20.40 & -2.13 & -3.62 & 0.59 & -0.54 & -13.10 & -5.53 & -0.40 & -0.03 & 0.62 \\
\hline Diversif & -3.44 & -2.13 & 15.70 & 1.47 & -0.13 & 0.28 & 6.06 & 1.98 & -0.09 & -1.35 & -5.00 \\
\hline Body Size & 5.02 & -3.62 & 1.47 & 31.40 & -0.83 & 0.01 & -1.87 & -8.30 & 0.10 & 0.19 & -1.80 \\
\hline Elev & -0.46 & 0.59 & -0.13 & -0.83 & 1.45 & -0.08 & 4.94 & 3.77 & 0.06 & -0.26 & 0.94 \\
\hline Lat & 0.16 & -0.54 & 0.28 & 0.01 & -0.08 & 0.40 & 0.66 & -1.15 & -0.22 & 0.23 & -0.26 \\
\hline Temp & -27.00 & -13.10 & 6.06 & -1.87 & 4.94 & 0.66 & 148.00 & 19.20 & -9.44 & -14.80 & 14.90 \\
\hline$H R$ & -4.56 & -5.53 & 1.98 & -8.30 & 3.77 & -1.15 & 19.20 & 222.00 & 2.97 & -17.10 & 12.60 \\
\hline NPP & 2.35 & -0.40 & -0.09 & 0.10 & 0.06 & -0.22 & -9.44 & 2.97 & 4.35 & 0.76 & -3.85 \\
\hline Prec & 4.47 & -0.03 & -1.35 & 0.19 & -0.26 & 0.23 & -14.80 & -17.10 & 0.76 & 6.92 & 2.99 \\
\hline UVB & 4.23 & 0.62 & -5.00 & -1.80 & 0.94 & -0.26 & 14.90 & 12.60 & -3.85 & 2.99 & 89.70 \\
\hline Partial C.C. & dS & $d N$ & Diversif & Body Size & Elev & Lat & Temp & HR & NPP & Prec & UVB \\
\hline $\mathrm{dS}$ & -1.00 & 0.31 & 0.20 & -0.20 & 0.09 & -0.06 & 0.52 & 0.07 & -0.27 & -0.40 & -0.10 \\
\hline $\mathrm{dN}$ & 0.31 & -1.00 & 0.12 & 0.15 & -0.11 & 0.22 & 0.22 & 0.08 & 0.04 & -0.01 & -0.01 \\
\hline Diversif & 0.20 & 0.12 & -1.00 & -0.07 & 0.03 & -0.11 & -0.12 & -0.03 & 0.01 & 0.12 & 0.13 \\
\hline Body Size & -0.20 & 0.15 & -0.07 & -1.00 & 0.12 & 0.00 & 0.03 & 0.10 & 0.00 & -0.01 & 0.04 \\
\hline Elev & 0.09 & -0.11 & 0.03 & 0.12 & -1.00 & 0.11 & -0.34 & -0.21 & -0.03 & 0.08 & -0.08 \\
\hline Lat & -0.06 & 0.22 & -0.11 & 0.00 & 0.11 & -1.00 & -0.08 & 0.13 & 0.17 & -0.14 & 0.04 \\
\hline Temp & 0.52 & 0.22 & -0.12 & 0.03 & -0.34 & -0.08 & -1.00 & -0.10 & 0.37 & 0.45 & -0.14 \\
\hline $\mathrm{HR}$ & 0.07 & 0.08 & -0.03 & 0.10 & -0.21 & 0.13 & -0.10 & -1.00 & -0.10 & 0.43 & -0.09 \\
\hline NPP & -0.27 & 0.04 & 0.01 & 0.00 & -0.03 & 0.17 & 0.37 & -0.10 & -1.00 & -0.13 & 0.20 \\
\hline Prec & -0.40 & -0.01 & 0.12 & -0.01 & 0.08 & -0.14 & 0.45 & 0.43 & -0.13 & -1.00 & -0.12 \\
\hline UVB & -0.10 & -0.01 & 0.13 & 0.04 & -0.08 & 0.04 & -0.14 & -0.09 & 0.20 & -0.12 & -1.00 \\
\hline Post. Prob. & dS & $d N$ & Diversif & Body Size & Elev & Lat & Temp & HR & NPP & Prec & UVB \\
\hline dS & - & 0.89 & 0.89 & 0.13 & 0.68 & 0.38 & 0.99 & 0.64 & 0.10 & 0.02 & 0.30 \\
\hline$d N$ & 0.89 & - & 0.73 & 0.75 & 0.32 & 0.80 & 0.82 & 0.62 & 0.57 & 0.48 & 0.49 \\
\hline Diversif & 0.89 & 0.73 & - & 0.29 & 0.59 & 0.19 & 0.21 & 0.41 & 0.52 & 0.84 & 0.87 \\
\hline Body Size & 0.13 & 0.75 & 0.29 & - & 0.84 & 0.51 & 0.57 & 0.80 & 0.49 & 0.48 & 0.62 \\
\hline Elev & 0.68 & 0.32 & 0.59 & 0.84 & - & 0.81 & 0.02 & 0.05 & 0.42 & 0.73 & 0.24 \\
\hline Lat & 0.38 & 0.80 & 0.19 & 0.51 & 0.81 & - & 0.32 & 0.83 & 0.89 & 0.17 & 0.64 \\
\hline Temp & 0.99 & 0.82 & 0.21 & 0.57 & 0.02 & 0.32 & - & 0.26 & 0.98 & 1.00 & 0.19 \\
\hline $\mathrm{HR}$ & 0.64 & 0.62 & 0.41 & 0.80 & 0.05 & 0.83 & 0.26 & - & 0.22 & 1.00 & 0.22 \\
\hline NPP & 0.10 & 0.57 & 0.52 & 0.49 & 0.42 & 0.89 & 0.98 & 0.22 & - & 0.18 & 0.94 \\
\hline Prec & 0.02 & 0.48 & 0.84 & 0.48 & 0.73 & 0.17 & 1.00 & 1.00 & 0.18 & - & 0.19 \\
\hline UVB & 0.30 & 0.49 & 0.87 & 0.62 & 0.24 & 0.64 & 0.19 & 0.22 & 0.94 & 0.19 & - \\
\hline
\end{tabular}




\begin{tabular}{|c|c|c|c|c|c|c|c|c|c|c|}
\hline \multicolumn{11}{|c|}{ MITOCHONDRIAL CODING-GENE: dN ANALYSIS 1 (Controlling for Temperature) } \\
\hline Covariances & dS & $d N$ & Diversif & Body Size & Elev & Lat & HR & NPP & Prec & UVB \\
\hline dS & 0.16 & 0.06 & 0.02 & -0.02 & 0.02 & -0.03 & 0.00 & -0.07 & -0.08 & -0.01 \\
\hline $\mathrm{dN}$ & 0.06 & 0.25 & 0.02 & 0.01 & -0.03 & 0.18 & 0.00 & 0.00 & -0.03 & 0.00 \\
\hline Diversif & 0.02 & 0.02 & 0.08 & -0.01 & 0.00 & -0.05 & 0.00 & -0.01 & 0.00 & 0.00 \\
\hline Body Size & -0.02 & 0.01 & -0.01 & 0.04 & 0.01 & 0.03 & 0.00 & 0.01 & 0.01 & 0.00 \\
\hline Elev & 0.02 & -0.03 & 0.00 & 0.01 & 0.94 & 0.09 & -0.02 & -0.01 & -0.01 & -0.01 \\
\hline Lat & -0.03 & 0.18 & -0.05 & 0.03 & 0.09 & 3.76 & 0.01 & 0.28 & -0.15 & 0.03 \\
\hline $\mathrm{HR}$ & 0.00 & 0.00 & 0.00 & 0.00 & -0.02 & 0.01 & 0.01 & -0.01 & 0.02 & 0.00 \\
\hline NPP & -0.07 & 0.00 & -0.01 & 0.01 & -0.01 & 0.28 & -0.01 & 0.38 & -0.05 & 0.02 \\
\hline Prec & -0.08 & -0.03 & 0.00 & 0.01 & -0.01 & -0.15 & 0.02 & -0.05 & 0.31 & -0.01 \\
\hline UVB & -0.01 & 0.00 & 0.00 & 0.00 & -0.01 & 0.03 & 0.00 & 0.02 & -0.01 & 0.02 \\
\hline Correl. Coeff. & dS & $\mathrm{dN}$ & Diversif & Body Size & Elev & Lat & HR & NPP & Prec & UVB \\
\hline dS & 1.00 & 0.30 & 0.22 & -0.20 & 0.05 & -0.03 & -0.07 & -0.26 & -0.35 & -0.10 \\
\hline $\mathrm{dN}$ & 0.30 & 1.00 & 0.13 & 0.06 & -0.07 & 0.19 & 0.07 & -0.01 & -0.11 & 0.00 \\
\hline Diversif & 0.22 & 0.13 & 1.00 & -0.10 & 0.01 & -0.10 & -0.01 & -0.06 & 0.01 & 0.08 \\
\hline Body Size & -0.20 & 0.06 & -0.10 & 1.00 & 0.07 & 0.06 & 0.12 & 0.07 & 0.11 & 0.04 \\
\hline Elev & 0.05 & -0.07 & 0.01 & 0.07 & 1.00 & 0.05 & -0.18 & -0.02 & -0.02 & -0.06 \\
\hline Lat & -0.03 & 0.19 & -0.10 & 0.06 & 0.05 & 1.00 & 0.05 & 0.23 & -0.13 & 0.10 \\
\hline $\mathrm{HR}$ & -0.07 & 0.07 & -0.01 & 0.12 & -0.18 & 0.05 & 1.00 & -0.18 & 0.47 & -0.18 \\
\hline NPP & -0.26 & -0.01 & -0.06 & 0.07 & -0.02 & 0.23 & -0.18 & 1.00 & -0.15 & 0.30 \\
\hline Prec & -0.35 & -0.11 & 0.01 & 0.11 & -0.02 & -0.13 & 0.47 & -0.15 & 1.00 & -0.17 \\
\hline UVB & -0.10 & 0.00 & 0.08 & 0.04 & -0.06 & 0.10 & -0.18 & 0.30 & -0.17 & 1.00 \\
\hline Post. Prob. & dS & $d N$ & Diversif & Body Size & Elev & Lat & HR & NPP & Prec & UVB \\
\hline dS & - & 0.86 & 0.95 & 0.09 & 0.62 & 0.43 & 0.34 & 0.08 & 0.03 & 0.27 \\
\hline $\mathrm{dN}$ & 0.86 & - & 0.79 & 0.62 & 0.36 & 0.78 & 0.61 & 0.49 & 0.33 & 0.50 \\
\hline Diversif & 0.95 & 0.79 & - & 0.17 & 0.55 & 0.17 & 0.46 & 0.28 & 0.55 & 0.77 \\
\hline Body Size & 0.09 & 0.62 & 0.17 & - & 0.75 & 0.73 & 0.88 & 0.73 & 0.84 & 0.64 \\
\hline Elev & 0.62 & 0.36 & 0.55 & 0.75 & - & 0.68 & 0.04 & 0.41 & 0.42 & 0.27 \\
\hline Lat & 0.43 & 0.78 & 0.17 & 0.73 & 0.68 & - & 0.67 & 0.99 & 0.10 & 0.83 \\
\hline $\mathrm{HR}$ & 0.34 & 0.61 & 0.46 & 0.88 & 0.04 & 0.67 & - & 0.04 & 1.00 & 0.04 \\
\hline NPP & 0.08 & 0.49 & 0.28 & 0.73 & 0.41 & 0.99 & 0.04 & - & 0.08 & 1.00 \\
\hline Prec & 0.03 & 0.33 & 0.55 & 0.84 & 0.42 & 0.10 & 1.00 & 0.08 & - & 0.05 \\
\hline UVB & 0.27 & 0.50 & 0.77 & 0.64 & 0.27 & 0.83 & 0.04 & 1.00 & 0.05 & - \\
\hline
\end{tabular}




\begin{tabular}{|c|c|c|c|c|c|c|c|c|c|c|c|}
\hline \multicolumn{12}{|c|}{ MITOCHONDRIAL CODING-GENE: dN ANALYSIS 2} \\
\hline Covariances & dS & $d \mathbf{N}$ & Diversif & Body Size & Elev & Lat & Temp & HR & NPP & Prec & UVB \\
\hline dS & 0.25 & 0.14 & 0.04 & -0.02 & -0.16 & -0.11 & 0.05 & 0.00 & -0.02 & -0.01 & -0.02 \\
\hline $\mathrm{dN}$ & 0.14 & 0.32 & 0.03 & 0.00 & -0.20 & 0.11 & 0.05 & 0.01 & 0.04 & 0.04 & -0.01 \\
\hline Diversif & 0.04 & 0.03 & 0.09 & -0.01 & -0.02 & -0.07 & 0.01 & 0.00 & -0.01 & 0.01 & 0.00 \\
\hline Body Size & -0.02 & 0.00 & -0.01 & 0.04 & 0.03 & 0.03 & 0.00 & 0.00 & 0.01 & 0.01 & 0.00 \\
\hline Elev & -0.16 & -0.20 & -0.02 & 0.03 & 1.36 & 0.28 & -0.11 & -0.03 & -0.11 & -0.17 & 0.02 \\
\hline Lat & -0.11 & 0.11 & -0.07 & 0.03 & 0.28 & 3.90 & -0.05 & 0.00 & 0.24 & -0.22 & 0.04 \\
\hline Temp & 0.05 & 0.05 & 0.01 & 0.00 & -0.11 & -0.05 & 0.03 & 0.00 & 0.03 & 0.05 & -0.01 \\
\hline HR & 0.00 & 0.01 & 0.00 & 0.00 & -0.03 & 0.00 & 0.00 & 0.01 & -0.01 & 0.03 & 0.00 \\
\hline NPP & -0.02 & 0.04 & -0.01 & 0.01 & -0.11 & 0.24 & 0.03 & -0.01 & 0.41 & -0.02 & 0.02 \\
\hline Prec & -0.01 & 0.04 & 0.01 & 0.01 & -0.17 & -0.22 & 0.05 & 0.03 & -0.02 & 0.38 & -0.02 \\
\hline UVB & -0.02 & -0.01 & 0.00 & 0.00 & 0.02 & 0.04 & -0.01 & 0.00 & 0.02 & -0.02 & 0.02 \\
\hline Correl. Coeff. & dS & $d N$ & Diversif & Body Size & Elev & Lat & Temp & HR & NPP & Prec & UVB \\
\hline dS & 1.00 & 0.50 & 0.24 & -0.21 & -0.29 & -0.11 & 0.58 & 0.10 & -0.07 & -0.02 & -0.26 \\
\hline $\mathrm{dN}$ & 0.50 & 1.00 & 0.17 & 0.00 & -0.33 & 0.09 & 0.50 & 0.19 & 0.12 & 0.12 & -0.16 \\
\hline Diversif & 0.24 & 0.17 & 1.00 & -0.11 & -0.05 & -0.11 & 0.11 & 0.02 & -0.03 & 0.06 & 0.03 \\
\hline Body Size & -0.21 & 0.00 & -0.11 & 1.00 & 0.11 & 0.08 & -0.09 & 0.09 & 0.04 & 0.06 & 0.06 \\
\hline Elev & -0.29 & -0.33 & -0.05 & 0.11 & 1.00 & 0.12 & -0.55 & -0.29 & -0.14 & -0.24 & 0.12 \\
\hline Lat & -0.11 & 0.09 & -0.11 & 0.08 & 0.12 & 1.00 & -0.14 & 0.01 & 0.19 & -0.18 & 0.14 \\
\hline Temp & 0.58 & 0.50 & 0.11 & -0.09 & -0.55 & -0.14 & 1.00 & 0.27 & 0.22 & 0.42 & -0.32 \\
\hline $\mathrm{HR}$ & 0.10 & 0.19 & 0.02 & 0.09 & -0.29 & 0.01 & 0.27 & 1.00 & -0.11 & 0.52 & -0.25 \\
\hline NPP & -0.07 & 0.12 & -0.03 & 0.04 & -0.14 & 0.19 & 0.22 & -0.11 & 1.00 & -0.04 & 0.21 \\
\hline Prec & -0.02 & 0.12 & 0.06 & 0.06 & -0.24 & -0.18 & 0.42 & 0.52 & -0.04 & 1.00 & -0.28 \\
\hline UVB & -0.26 & -0.16 & 0.03 & 0.06 & 0.12 & 0.14 & -0.32 & -0.25 & 0.21 & -0.28 & 1.00 \\
\hline Post. Prob. & dS & $d N$ & Diversif & Body Size & Elev & Lat & Temp & HR & NPP & Prec & UVB \\
\hline dS & - & 0.98 & 0.98 & 0.06 & 0.03 & 0.25 & 1.00 & 0.73 & 0.34 & 0.45 & 0.04 \\
\hline $\mathrm{dN}$ & 0.98 & - & 0.87 & 0.49 & 0.05 & 0.65 & 0.99 & 0.81 & 0.70 & 0.70 & 0.22 \\
\hline Diversif & 0.98 & 0.87 & - & 0.15 & 0.30 & 0.14 & 0.86 & 0.59 & 0.38 & 0.71 & 0.63 \\
\hline Body Size & 0.06 & 0.49 & 0.15 & - & 0.85 & 0.77 & 0.19 & 0.80 & 0.66 & 0.71 & 0.73 \\
\hline Elev & 0.03 & 0.05 & 0.30 & 0.85 & - & 0.87 & 0.00 & 0.00 & 0.09 & 0.01 & 0.88 \\
\hline Lat & 0.25 & 0.65 & 0.14 & 0.77 & 0.87 & - & 0.09 & 0.53 & 0.96 & 0.04 & 0.91 \\
\hline Temp & 1.00 & 0.99 & 0.86 & 0.19 & 0.00 & 0.09 & - & 1.00 & 0.98 & 1.00 & 0.00 \\
\hline $\mathrm{HR}$ & 0.73 & 0.81 & 0.59 & 0.80 & 0.00 & 0.53 & 1.00 & - & 0.15 & 1.00 & 0.01 \\
\hline NPP & 0.34 & 0.70 & 0.38 & 0.66 & 0.09 & 0.96 & 0.98 & 0.15 & - & 0.35 & 0.98 \\
\hline Prec & 0.45 & 0.70 & 0.71 & 0.71 & 0.01 & 0.04 & 1.00 & 1.00 & 0.35 & - & 0.00 \\
\hline UVB & 0.04 & 0.22 & 0.63 & 0.73 & 0.88 & 0.91 & 0.00 & 0.01 & 0.98 & 0.00 & - \\
\hline
\end{tabular}




\begin{tabular}{|c|c|c|c|c|c|c|c|c|c|c|c|}
\hline Precisions & dS & $d N$ & Diversif & Body Size & Elev & Lat & Temp & HR & NPP & Prec & UVB \\
\hline dS & 18.70 & -6.07 & -3.56 & 4.98 & -0.48 & 0.17 & -27.70 & -4.66 & 2.30 & 4.56 & 4.41 \\
\hline $\mathrm{dN}$ & -6.07 & 35.40 & -2.25 & -3.59 & 0.63 & -0.55 & -14.70 & -5.63 & -0.37 & 0.01 & 0.48 \\
\hline Diversif & -3.56 & -2.25 & 15.80 & 1.48 & -0.12 & 0.28 & 6.53 & 2.04 & -0.08 & -1.39 & -4.91 \\
\hline Body Size & 4.98 & -3.59 & 1.48 & 31.30 & -0.82 & 0.01 & -1.97 & -8.32 & 0.05 & 0.20 & -1.59 \\
\hline Elev & -0.48 & 0.63 & -0.12 & -0.82 & 1.44 & -0.08 & 4.92 & 3.76 & 0.07 & -0.27 & 0.92 \\
\hline Lat & 0.17 & -0.55 & 0.28 & 0.01 & -0.08 & 0.40 & 0.65 & -1.15 & -0.22 & 0.23 & -0.26 \\
\hline Temp & -27.70 & -14.70 & 6.53 & -1.97 & 4.92 & 0.65 & 150.00 & 19.80 & -9.36 & -15.00 & 14.60 \\
\hline$H R$ & -4.66 & -5.63 & 2.04 & -8.32 & 3.76 & -1.15 & 19.80 & 220.00 & 2.96 & -17.10 & 12.80 \\
\hline NPP & 2.30 & -0.37 & -0.08 & 0.05 & 0.07 & -0.22 & -9.36 & 2.96 & 4.32 & 0.75 & -3.85 \\
\hline Prec & 4.56 & 0.01 & -1.39 & 0.20 & -0.27 & 0.23 & -15.00 & -17.10 & 0.75 & 6.94 & 3.02 \\
\hline UVB & 4.41 & 0.48 & -4.91 & -1.59 & 0.92 & -0.26 & 14.60 & 12.80 & -3.85 & 3.02 & 89.40 \\
\hline Partial C.C. & dS & $d N$ & Diversif & Body Size & Elev & Lat & Temp & HR & NPP & Prec & UVB \\
\hline dS & -1.00 & 0.31 & 0.21 & -0.19 & 0.09 & -0.07 & 0.52 & 0.08 & -0.26 & -0.40 & -0.10 \\
\hline $\mathrm{dN}$ & 0.31 & -1.00 & 0.12 & 0.14 & -0.11 & 0.22 & 0.22 & 0.08 & 0.04 & -0.01 & -0.01 \\
\hline Diversif & 0.21 & 0.12 & -1.00 & -0.07 & 0.03 & -0.11 & -0.12 & -0.03 & 0.01 & 0.13 & 0.13 \\
\hline Body Size & -0.19 & 0.14 & -0.07 & -1.00 & 0.12 & 0.00 & 0.03 & 0.10 & 0.00 & -0.01 & 0.03 \\
\hline Elev & 0.09 & -0.11 & 0.03 & 0.12 & -1.00 & 0.11 & -0.34 & -0.21 & -0.03 & 0.08 & -0.08 \\
\hline Lat & -0.07 & 0.22 & -0.11 & 0.00 & 0.11 & -1.00 & -0.08 & 0.13 & 0.17 & -0.14 & 0.04 \\
\hline Temp & 0.52 & 0.22 & -0.12 & 0.03 & -0.34 & -0.08 & -1.00 & -0.10 & 0.36 & 0.46 & -0.13 \\
\hline$H R$ & 0.08 & 0.08 & -0.03 & 0.10 & -0.21 & 0.13 & -0.10 & -1.00 & -0.10 & 0.44 & -0.09 \\
\hline NPP & -0.26 & 0.04 & 0.01 & 0.00 & -0.03 & 0.17 & 0.36 & -0.10 & -1.00 & -0.13 & 0.20 \\
\hline Prec & -0.40 & -0.01 & 0.13 & -0.01 & 0.08 & -0.14 & 0.46 & 0.44 & -0.13 & -1.00 & -0.12 \\
\hline UVB & -0.10 & -0.01 & 0.13 & 0.03 & -0.08 & 0.04 & -0.13 & -0.09 & 0.20 & -0.12 & -1.00 \\
\hline Post. Prob. & dS & $d N$ & Diversif & Body Size & Elev & Lat & Temp & HR & NPP & Prec & UVB \\
\hline dS & - & 0.88 & 0.90 & 0.15 & 0.68 & 0.37 & 0.99 & 0.65 & 0.10 & 0.02 & 0.30 \\
\hline $\mathrm{dN}$ & 0.88 & - & 0.74 & 0.74 & 0.32 & 0.80 & 0.82 & 0.62 & 0.57 & 0.47 & 0.49 \\
\hline Diversif & 0.90 & 0.74 & - & 0.29 & 0.58 & 0.20 & 0.20 & 0.40 & 0.53 & 0.84 & 0.86 \\
\hline Body Size & 0.15 & 0.74 & 0.29 & - & 0.84 & 0.51 & 0.57 & 0.80 & 0.51 & 0.48 & 0.61 \\
\hline Elev & 0.68 & 0.32 & 0.58 & 0.84 & - & 0.81 & 0.02 & 0.04 & 0.41 & 0.73 & 0.24 \\
\hline Lat & 0.37 & 0.80 & 0.20 & 0.51 & 0.81 & - & 0.32 & 0.84 & 0.88 & 0.16 & 0.64 \\
\hline Temp & 0.99 & 0.82 & 0.20 & 0.57 & 0.02 & 0.32 & - & 0.26 & 0.98 & 1.00 & 0.20 \\
\hline $\mathrm{HR}$ & 0.65 & 0.62 & 0.40 & 0.80 & 0.04 & 0.84 & 0.26 & - & 0.22 & 1.00 & 0.21 \\
\hline NPP & 0.10 & 0.57 & 0.53 & 0.51 & 0.41 & 0.88 & 0.98 & 0.22 & - & 0.18 & 0.94 \\
\hline Prec & 0.02 & 0.47 & 0.84 & 0.48 & 0.73 & 0.16 & 1.00 & 1.00 & 0.18 & - & 0.19 \\
\hline UVB & 0.30 & 0.49 & 0.86 & 0.61 & 0.24 & 0.64 & 0.20 & 0.21 & 0.94 & 0.19 & - \\
\hline
\end{tabular}




\begin{tabular}{|c|c|c|c|c|c|c|c|c|c|c|}
\hline \multicolumn{11}{|c|}{ MITOCHONDRIAL CODING-GENE: dN ANALYSIS 2 (Controlling for Temperature) } \\
\hline Covariances & dS & $d \mathbf{N}$ & Diversif & Body Size & Elev & Lat & HR & NPP & Prec & UVB \\
\hline dS & 0.16 & 0.06 & 0.03 & -0.02 & 0.02 & -0.03 & 0.00 & -0.06 & -0.08 & -0.01 \\
\hline $\mathrm{dN}$ & 0.06 & 0.23 & 0.02 & 0.00 & -0.03 & 0.18 & 0.00 & 0.00 & -0.03 & 0.00 \\
\hline Diversif & 0.03 & 0.02 & 0.08 & -0.01 & 0.00 & -0.06 & 0.00 & -0.01 & 0.00 & 0.00 \\
\hline Body Size & -0.02 & 0.00 & -0.01 & 0.04 & 0.01 & 0.03 & 0.00 & 0.01 & 0.01 & 0.00 \\
\hline Elev & 0.02 & -0.03 & 0.00 & 0.01 & 0.94 & 0.10 & -0.02 & -0.02 & -0.01 & -0.01 \\
\hline Lat & -0.03 & 0.18 & -0.06 & 0.03 & 0.10 & 3.78 & 0.01 & 0.28 & -0.15 & 0.03 \\
\hline $\mathrm{HR}$ & 0.00 & 0.00 & 0.00 & 0.00 & -0.02 & 0.01 & 0.01 & -0.01 & 0.02 & 0.00 \\
\hline NPP & -0.06 & 0.00 & -0.01 & 0.01 & -0.02 & 0.28 & -0.01 & 0.38 & -0.05 & 0.02 \\
\hline Prec & -0.08 & -0.03 & 0.00 & 0.01 & -0.01 & -0.15 & 0.02 & -0.05 & 0.31 & -0.01 \\
\hline UVB & -0.01 & 0.00 & 0.00 & 0.00 & -0.01 & 0.03 & 0.00 & 0.02 & -0.01 & 0.02 \\
\hline Correl. Coeff. & dS & $d \mathbf{N}$ & Diversif & Body Size & Elev & Lat & HR & NPP & Prec & UVB \\
\hline $\mathrm{dS}$ & 1.00 & 0.30 & 0.22 & -0.20 & 0.05 & -0.03 & -0.07 & -0.25 & -0.35 & -0.10 \\
\hline $\mathrm{dN}$ & 0.30 & 1.00 & 0.13 & 0.05 & -0.07 & 0.19 & 0.07 & 0.00 & -0.11 & 0.00 \\
\hline Diversif & 0.22 & 0.13 & 1.00 & -0.10 & 0.01 & -0.10 & -0.01 & -0.06 & 0.01 & 0.08 \\
\hline Body Size & -0.20 & 0.05 & -0.10 & 1.00 & 0.07 & 0.06 & 0.12 & 0.07 & 0.11 & 0.04 \\
\hline Elev & 0.05 & -0.07 & 0.01 & 0.07 & 1.00 & 0.05 & -0.18 & -0.03 & -0.02 & -0.06 \\
\hline Lat & -0.03 & 0.19 & -0.10 & 0.06 & 0.05 & 1.00 & 0.05 & 0.23 & -0.13 & 0.10 \\
\hline$H R$ & -0.07 & 0.07 & -0.01 & 0.12 & -0.18 & 0.05 & 1.00 & -0.18 & 0.47 & -0.19 \\
\hline NPP & -0.25 & 0.00 & -0.06 & 0.07 & -0.03 & 0.23 & -0.18 & 1.00 & -0.15 & 0.30 \\
\hline Prec & -0.35 & -0.11 & 0.01 & 0.11 & -0.02 & -0.13 & 0.47 & -0.15 & 1.00 & -0.17 \\
\hline UVB & -0.10 & 0.00 & 0.08 & 0.04 & -0.06 & 0.10 & -0.19 & 0.30 & -0.17 & 1.00 \\
\hline Post. Prob. & dS & $d N$ & Diversif & Body Size & Elev & Lat & HR & NPP & Prec & UVB \\
\hline dS & - & 0.86 & 0.95 & 0.10 & 0.61 & 0.43 & 0.34 & 0.09 & 0.02 & 0.27 \\
\hline $\mathrm{dN}$ & 0.86 & - & 0.79 & 0.61 & 0.37 & 0.78 & 0.62 & 0.49 & 0.32 & 0.50 \\
\hline Diversif & 0.95 & 0.79 & - & 0.17 & 0.54 & 0.17 & 0.46 & 0.28 & 0.55 & 0.76 \\
\hline Body Size & 0.10 & 0.61 & 0.17 & - & 0.75 & 0.73 & 0.87 & 0.73 & 0.84 & 0.63 \\
\hline Elev & 0.61 & 0.37 & 0.54 & 0.75 & - & 0.69 & 0.04 & 0.41 & 0.43 & 0.27 \\
\hline Lat & 0.43 & 0.78 & 0.17 & 0.73 & 0.69 & - & 0.67 & 0.99 & 0.10 & 0.84 \\
\hline$H R$ & 0.34 & 0.62 & 0.46 & 0.87 & 0.04 & 0.67 & - & 0.04 & 1.00 & 0.04 \\
\hline NPP & 0.09 & 0.49 & 0.28 & 0.73 & 0.41 & 0.99 & 0.04 & - & 0.07 & 1.00 \\
\hline Prec & 0.02 & 0.32 & 0.55 & 0.84 & 0.43 & 0.10 & 1.00 & 0.07 & - & 0.05 \\
\hline UVB & 0.27 & 0.50 & 0.76 & 0.63 & 0.27 & 0.84 & 0.04 & 1.00 & 0.05 & - \\
\hline
\end{tabular}




\begin{tabular}{|c|c|c|c|c|c|c|c|c|c|c|c|}
\hline \multicolumn{12}{|c|}{ MITOCHONDRIAL CODING-GENE: $\omega$ ANALYSIS 1} \\
\hline Covariances & dS & $\omega$ & Diversif & Body Size & Elev & Lat & Temp & HR & NPP & Prec & UVB \\
\hline $\mathrm{dS}$ & 0.27 & 0.00 & 0.03 & -0.02 & -0.17 & -0.07 & 0.05 & 0.00 & -0.02 & -0.01 & -0.02 \\
\hline$\omega$ & 0.00 & 0.04 & 0.00 & 0.00 & 0.00 & 0.02 & 0.00 & 0.00 & 0.00 & 0.00 & 0.00 \\
\hline Diversif & 0.03 & 0.00 & 0.09 & -0.01 & -0.02 & -0.07 & 0.01 & 0.00 & -0.01 & 0.01 & 0.00 \\
\hline Body Size & -0.02 & 0.00 & -0.01 & 0.04 & 0.03 & 0.03 & 0.00 & 0.00 & 0.01 & 0.01 & 0.00 \\
\hline Elev & -0.17 & 0.00 & -0.02 & 0.03 & 1.33 & 0.28 & -0.11 & -0.03 & -0.10 & -0.17 & 0.02 \\
\hline Lat & -0.07 & 0.02 & -0.07 & 0.03 & 0.28 & 3.85 & -0.05 & 0.00 & 0.24 & -0.22 & 0.04 \\
\hline Temp & 0.05 & 0.00 & 0.01 & 0.00 & -0.11 & -0.05 & 0.03 & 0.00 & 0.02 & 0.05 & -0.01 \\
\hline $\mathrm{HR}$ & 0.00 & 0.00 & 0.00 & 0.00 & -0.03 & 0.00 & 0.00 & 0.01 & -0.01 & 0.03 & 0.00 \\
\hline NPP & -0.02 & 0.00 & -0.01 & 0.01 & -0.10 & 0.24 & 0.02 & -0.01 & 0.40 & -0.02 & 0.02 \\
\hline Prec & -0.01 & 0.00 & 0.01 & 0.01 & -0.17 & -0.22 & 0.05 & 0.03 & -0.02 & 0.37 & -0.02 \\
\hline UVB & -0.02 & 0.00 & 0.00 & 0.00 & 0.02 & 0.04 & -0.01 & 0.00 & 0.02 & -0.02 & 0.02 \\
\hline Correl. Coeff. & dS & $\omega$ & Diversif & Body Size & Elev & Lat & Temp & HR & NPP & Prec & UVB \\
\hline dS & 1.00 & -0.03 & 0.23 & -0.20 & -0.29 & -0.07 & 0.57 & 0.10 & -0.06 & -0.02 & -0.25 \\
\hline$\omega$ & -0.03 & 1.00 & 0.01 & 0.08 & 0.02 & 0.03 & -0.03 & 0.03 & -0.01 & 0.02 & 0.01 \\
\hline Diversif & 0.23 & 0.01 & 1.00 & -0.11 & -0.05 & -0.12 & 0.11 & 0.02 & -0.03 & 0.06 & 0.04 \\
\hline Body Size & -0.20 & 0.08 & -0.11 & 1.00 & 0.11 & 0.08 & -0.10 & 0.09 & 0.04 & 0.05 & 0.07 \\
\hline Elev & -0.29 & 0.02 & -0.05 & 0.11 & 1.00 & 0.12 & -0.55 & -0.30 & -0.14 & -0.25 & 0.12 \\
\hline Lat & -0.07 & 0.03 & -0.12 & 0.08 & 0.12 & 1.00 & -0.15 & 0.00 & 0.19 & -0.18 & 0.14 \\
\hline Temp & 0.57 & -0.03 & 0.11 & -0.10 & -0.55 & -0.15 & 1.00 & 0.27 & 0.22 & 0.41 & -0.31 \\
\hline$H R$ & 0.10 & 0.03 & 0.02 & 0.09 & -0.30 & 0.00 & 0.27 & 1.00 & -0.11 & 0.53 & -0.25 \\
\hline NPP & -0.06 & -0.01 & -0.03 & 0.04 & -0.14 & 0.19 & 0.22 & -0.11 & 1.00 & -0.05 & 0.21 \\
\hline Prec & -0.02 & 0.02 & 0.06 & 0.05 & -0.25 & -0.18 & 0.41 & 0.53 & -0.05 & 1.00 & -0.28 \\
\hline UVB & -0.25 & 0.01 & 0.04 & 0.07 & 0.12 & 0.14 & -0.31 & -0.25 & 0.21 & -0.28 & 1.00 \\
\hline Post. Prob. & dS & $\omega$ & Diversif & Body Size & Elev & Lat & Temp & HR & NPP & Prec & UVB \\
\hline dS & - & 0.46 & 0.97 & 0.08 & 0.02 & 0.32 & 1.00 & 0.73 & 0.35 & 0.45 & 0.05 \\
\hline$\omega$ & 0.46 & - & 0.51 & 0.62 & 0.52 & 0.54 & 0.48 & 0.54 & 0.48 & 0.53 & 0.51 \\
\hline Diversif & 0.97 & 0.51 & - & 0.14 & 0.30 & 0.13 & 0.86 & 0.58 & 0.38 & 0.71 & 0.64 \\
\hline Body Size & 0.08 & 0.62 & 0.14 & - & 0.85 & 0.77 & 0.18 & 0.80 & 0.65 & 0.70 & 0.73 \\
\hline Elev & 0.02 & 0.52 & 0.30 & 0.85 & - & 0.88 & 0.00 & 0.00 & 0.09 & 0.01 & 0.87 \\
\hline Lat & 0.32 & 0.54 & 0.13 & 0.77 & 0.88 & - & 0.09 & 0.50 & 0.97 & 0.04 & 0.91 \\
\hline Temp & 1.00 & 0.48 & 0.86 & 0.18 & 0.00 & 0.09 & - & 0.99 & 0.98 & 1.00 & 0.00 \\
\hline $\mathrm{HR}$ & 0.73 & 0.54 & 0.58 & 0.80 & 0.00 & 0.50 & 0.99 & - & 0.15 & 1.00 & 0.01 \\
\hline NPP & 0.35 & 0.48 & 0.38 & 0.65 & 0.09 & 0.97 & 0.98 & 0.15 & - & 0.34 & 0.98 \\
\hline Prec & 0.45 & 0.53 & 0.71 & 0.70 & 0.01 & 0.04 & 1.00 & 1.00 & 0.34 & - & 0.00 \\
\hline UVB & 0.05 & 0.51 & 0.64 & 0.73 & 0.87 & 0.91 & 0.00 & 0.01 & 0.98 & 0.00 & - \\
\hline
\end{tabular}




\begin{tabular}{|c|c|c|c|c|c|c|c|c|c|c|c|}
\hline Precisions & dS & $\omega$ & Diversif & Body Size & Elev & Lat & Temp & $H R$ & NPP & Prec & UVB \\
\hline dS & 14.70 & 0.46 & -3.51 & 2.58 & -0.12 & -0.12 & -26.50 & -5.14 & 2.01 & 3.94 & 3.38 \\
\hline$\omega$ & 0.46 & 3280.00 & -5.22 & -18.90 & -0.88 & -0.37 & 15.10 & -14.70 & 2.15 & -0.78 & 1.09 \\
\hline Diversif & -3.51 & -5.22 & 16.50 & 1.41 & -0.09 & 0.24 & 3.54 & 0.97 & -0.11 & -1.15 & -4.58 \\
\hline Body Size & 2.58 & -18.90 & 1.41 & 33.20 & -0.61 & -0.11 & -2.68 & -8.34 & -0.19 & 0.04 & -2.04 \\
\hline Elev & -0.12 & -0.88 & -0.09 & -0.61 & 1.52 & -0.06 & 4.90 & 3.85 & 0.10 & -0.23 & 0.94 \\
\hline Lat & -0.12 & -0.37 & 0.24 & -0.11 & -0.06 & 0.40 & 0.51 & -1.29 & -0.25 & 0.19 & -0.25 \\
\hline Temp & -26.50 & 15.10 & 3.54 & -2.68 & 4.90 & 0.51 & 139.00 & 14.10 & -9.05 & -13.70 & 15.80 \\
\hline $\mathrm{HR}$ & -5.14 & -14.70 & 0.97 & -8.34 & 3.85 & -1.29 & 14.10 & 236.00 & 2.92 & -16.50 & 13.20 \\
\hline NPP & 2.01 & 2.15 & -0.11 & -0.19 & 0.10 & -0.25 & -9.05 & 2.92 & 4.56 & 0.72 & -3.84 \\
\hline Prec & 3.94 & -0.78 & -1.15 & 0.04 & -0.23 & 0.19 & -13.70 & -16.50 & 0.72 & 7.22 & 2.61 \\
\hline UVB & 3.38 & 1.09 & -4.58 & -2.04 & 0.94 & -0.25 & 15.80 & 13.20 & -3.84 & 2.61 & 94.40 \\
\hline Partial C.C. & dS & $\omega$ & Diversif & Body Size & Elev & Lat & Temp & HR & NPP & Prec & UVB \\
\hline dS & -1.00 & -0.01 & 0.22 & -0.12 & 0.03 & 0.05 & 0.58 & 0.09 & -0.25 & -0.38 & -0.09 \\
\hline$\omega$ & -0.01 & -1.00 & 0.03 & 0.11 & 0.01 & 0.03 & -0.02 & 0.03 & -0.02 & 0.02 & 0.01 \\
\hline Diversif & 0.22 & 0.03 & -1.00 & -0.06 & 0.02 & -0.09 & -0.07 & -0.01 & 0.01 & 0.10 & 0.12 \\
\hline Body Size & -0.12 & 0.11 & -0.06 & -1.00 & 0.09 & 0.03 & 0.04 & 0.10 & 0.02 & 0.00 & 0.04 \\
\hline Elev & 0.03 & 0.01 & 0.02 & 0.09 & -1.00 & 0.08 & -0.34 & -0.21 & -0.04 & 0.07 & -0.08 \\
\hline Lat & 0.05 & 0.03 & -0.09 & 0.03 & 0.08 & -1.00 & -0.07 & 0.14 & 0.19 & -0.12 & 0.04 \\
\hline Temp & 0.58 & -0.02 & -0.07 & 0.04 & -0.34 & -0.07 & -1.00 & -0.08 & 0.36 & 0.43 & -0.14 \\
\hline $\mathrm{HR}$ & 0.09 & 0.03 & -0.01 & 0.10 & -0.21 & 0.14 & -0.08 & -1.00 & -0.09 & 0.41 & -0.09 \\
\hline NPP & -0.25 & -0.02 & 0.01 & 0.02 & -0.04 & 0.19 & 0.36 & -0.09 & -1.00 & -0.12 & 0.19 \\
\hline Prec & -0.38 & 0.02 & 0.10 & 0.00 & 0.07 & -0.12 & 0.43 & 0.41 & -0.12 & -1.00 & -0.10 \\
\hline UVB & -0.09 & 0.01 & 0.12 & 0.04 & -0.08 & 0.04 & -0.14 & -0.09 & 0.19 & -0.10 & -1.00 \\
\hline Post. Prob. & dS & $\omega$ & Diversif & Body Size & Elev & Lat & Temp & $H R$ & NPP & Prec & UVB \\
\hline dS & - & 0.49 & 0.91 & 0.26 & 0.56 & 0.59 & 1.00 & 0.67 & 0.11 & 0.02 & 0.31 \\
\hline$\omega$ & 0.49 & - & 0.55 & 0.63 & 0.51 & 0.55 & 0.47 & 0.54 & 0.48 & 0.52 & 0.51 \\
\hline Diversif & 0.91 & 0.55 & - & 0.32 & 0.56 & 0.24 & 0.33 & 0.46 & 0.53 & 0.77 & 0.82 \\
\hline Body Size & 0.26 & 0.63 & 0.32 & - & 0.75 & 0.60 & 0.58 & 0.77 & 0.56 & 0.50 & 0.62 \\
\hline Elev & 0.56 & 0.51 & 0.56 & 0.75 & - & 0.74 & 0.02 & 0.07 & 0.38 & 0.69 & 0.26 \\
\hline Lat & 0.59 & 0.55 & 0.24 & 0.60 & 0.74 & - & 0.34 & 0.85 & 0.91 & 0.21 & 0.63 \\
\hline Temp & 1.00 & 0.47 & 0.33 & 0.58 & 0.02 & 0.34 & - & 0.32 & 0.98 & 0.99 & 0.18 \\
\hline $\mathrm{HR}$ & 0.67 & 0.54 & 0.46 & 0.77 & 0.07 & 0.85 & 0.32 & - & 0.25 & 0.99 & 0.24 \\
\hline NPP & 0.11 & 0.48 & 0.53 & 0.56 & 0.38 & 0.91 & 0.98 & 0.25 & - & 0.21 & 0.91 \\
\hline Prec & 0.02 & 0.52 & 0.77 & 0.50 & 0.69 & 0.21 & 0.99 & 0.99 & 0.21 & - & 0.24 \\
\hline UVB & 0.31 & 0.51 & 0.82 & 0.62 & 0.26 & 0.63 & 0.18 & 0.24 & 0.91 & 0.24 & - \\
\hline
\end{tabular}




\begin{tabular}{|c|c|c|c|c|c|c|c|c|c|c|}
\hline \multicolumn{11}{|c|}{ MITOCHONDRIAL CODING-GENE: $\omega$ ANALYSIS 1 (Controlling for Temperature) } \\
\hline Covariances & dS & $\omega$ & Diversif & Body Size & Elev & Lat & HR & NPP & Prec & UVB \\
\hline dS & 0.18 & 0.00 & 0.02 & -0.02 & 0.01 & 0.01 & 0.00 & -0.06 & -0.08 & 0.00 \\
\hline$\omega$ & 0.00 & 0.03 & 0.00 & 0.00 & 0.00 & 0.02 & 0.00 & 0.00 & 0.00 & 0.00 \\
\hline Diversif & 0.02 & 0.00 & 0.08 & -0.01 & 0.00 & -0.06 & 0.00 & -0.01 & 0.00 & 0.00 \\
\hline Body Size & -0.02 & 0.00 & -0.01 & 0.04 & 0.01 & 0.03 & 0.00 & 0.01 & 0.01 & 0.00 \\
\hline Elev & 0.01 & 0.00 & 0.00 & 0.01 & 0.92 & 0.10 & -0.02 & -0.02 & -0.01 & -0.01 \\
\hline Lat & 0.01 & 0.02 & -0.06 & 0.03 & 0.10 & 3.72 & 0.01 & 0.28 & -0.15 & 0.03 \\
\hline HR & 0.00 & 0.00 & 0.00 & 0.00 & -0.02 & 0.01 & 0.01 & -0.01 & 0.02 & 0.00 \\
\hline NPP & -0.06 & 0.00 & -0.01 & 0.01 & -0.02 & 0.28 & -0.01 & 0.38 & -0.05 & 0.02 \\
\hline Prec & -0.08 & 0.00 & 0.00 & 0.01 & -0.01 & -0.15 & 0.02 & -0.05 & 0.31 & -0.01 \\
\hline UVB & 0.00 & 0.00 & 0.00 & 0.00 & -0.01 & 0.03 & 0.00 & 0.02 & -0.01 & 0.02 \\
\hline Correl. Coeff. & dS & $\omega$ & Diversif & Body Size & Elev & Lat & HR & NPP & Prec & UVB \\
\hline dS & 1.00 & -0.02 & 0.21 & -0.17 & 0.03 & 0.01 & -0.07 & -0.24 & -0.35 & -0.09 \\
\hline$\omega$ & -0.02 & 1.00 & 0.01 & 0.09 & 0.00 & 0.03 & 0.04 & -0.01 & 0.03 & 0.00 \\
\hline Diversif & 0.21 & 0.01 & 1.00 & -0.10 & 0.01 & -0.10 & -0.01 & -0.06 & 0.01 & 0.08 \\
\hline Body Size & -0.17 & 0.09 & -0.10 & 1.00 & 0.07 & 0.06 & 0.12 & 0.06 & 0.11 & 0.04 \\
\hline Elev & 0.03 & 0.00 & 0.01 & 0.07 & 1.00 & 0.05 & -0.19 & -0.03 & -0.02 & -0.06 \\
\hline Lat & 0.01 & 0.03 & -0.10 & 0.06 & 0.05 & 1.00 & 0.04 & 0.23 & -0.14 & 0.10 \\
\hline$H R$ & -0.07 & 0.04 & -0.01 & 0.12 & -0.19 & 0.04 & 1.00 & -0.18 & 0.47 & -0.19 \\
\hline NPP & -0.24 & -0.01 & -0.06 & 0.06 & -0.03 & 0.23 & -0.18 & 1.00 & -0.15 & 0.30 \\
\hline Prec & -0.35 & 0.03 & 0.01 & 0.11 & -0.02 & -0.14 & 0.47 & -0.15 & 1.00 & -0.18 \\
\hline UVB & -0.09 & 0.00 & 0.08 & 0.04 & -0.06 & 0.10 & -0.19 & 0.30 & -0.18 & 1.00 \\
\hline Post. Prob. & dS & $\omega$ & Diversif & Body Size & Elev & Lat & HR & NPP & Prec & UVB \\
\hline dS & - & 0.47 & 0.94 & 0.13 & 0.57 & 0.52 & 0.35 & 0.10 & 0.02 & 0.29 \\
\hline$\omega$ & 0.47 & - & 0.52 & 0.63 & 0.51 & 0.54 & 0.55 & 0.49 & 0.55 & 0.50 \\
\hline Diversif & 0.94 & 0.52 & - & 0.17 & 0.53 & 0.16 & 0.46 & 0.29 & 0.54 & 0.77 \\
\hline Body Size & 0.13 & 0.63 & 0.17 & - & 0.73 & 0.74 & 0.87 & 0.73 & 0.84 & 0.64 \\
\hline Elev & 0.57 & 0.51 & 0.53 & 0.73 & - & 0.69 & 0.04 & 0.40 & 0.41 & 0.27 \\
\hline Lat & 0.52 & 0.54 & 0.16 & 0.74 & 0.69 & - & 0.66 & 0.99 & 0.10 & 0.83 \\
\hline$H R$ & 0.35 & 0.55 & 0.46 & 0.87 & 0.04 & 0.66 & - & 0.04 & 1.00 & 0.04 \\
\hline NPP & 0.10 & 0.49 & 0.29 & 0.73 & 0.40 & 0.99 & 0.04 & - & 0.07 & 1.00 \\
\hline Prec & 0.02 & 0.55 & 0.54 & 0.84 & 0.41 & 0.10 & 1.00 & 0.07 & - & 0.04 \\
\hline UVB & 0.29 & 0.50 & 0.77 & 0.64 & 0.27 & 0.83 & 0.04 & 1.00 & 0.04 & - \\
\hline
\end{tabular}




\begin{tabular}{|c|c|c|c|c|c|c|c|c|c|c|c|}
\hline \multicolumn{12}{|c|}{ MITOCHONDRIAL CODING-GENE: $\omega$ ANALYSIS 2} \\
\hline Covariances & dS & $\omega$ & Diversif & Body Size & Elev & Lat & Temp & HR & NPP & Prec & UVB \\
\hline dS & 0.27 & 0.00 & 0.04 & -0.02 & -0.17 & -0.07 & 0.05 & 0.00 & -0.02 & -0.01 & -0.02 \\
\hline$\omega$ & 0.00 & 0.04 & 0.00 & 0.00 & 0.00 & 0.02 & 0.00 & 0.00 & 0.00 & 0.00 & 0.00 \\
\hline Diversif & 0.04 & 0.00 & 0.09 & -0.01 & -0.02 & -0.07 & 0.01 & 0.00 & -0.01 & 0.01 & 0.00 \\
\hline Body Size & -0.02 & 0.00 & -0.01 & 0.04 & 0.03 & 0.03 & 0.00 & 0.00 & 0.01 & 0.01 & 0.00 \\
\hline Elev & -0.17 & 0.00 & -0.02 & 0.03 & 1.34 & 0.28 & -0.11 & -0.03 & -0.10 & -0.17 & 0.02 \\
\hline Lat & -0.07 & 0.02 & -0.07 & 0.03 & 0.28 & 3.84 & -0.05 & 0.00 & 0.24 & -0.22 & 0.04 \\
\hline Temp & 0.05 & 0.00 & 0.01 & 0.00 & -0.11 & -0.05 & 0.03 & 0.00 & 0.02 & 0.05 & -0.01 \\
\hline $\mathrm{HR}$ & 0.00 & 0.00 & 0.00 & 0.00 & -0.03 & 0.00 & 0.00 & 0.01 & -0.01 & 0.03 & 0.00 \\
\hline NPP & -0.02 & 0.00 & -0.01 & 0.01 & -0.10 & 0.24 & 0.02 & -0.01 & 0.40 & -0.02 & 0.02 \\
\hline Prec & -0.01 & 0.00 & 0.01 & 0.01 & -0.17 & -0.22 & 0.05 & 0.03 & -0.02 & 0.38 & -0.02 \\
\hline UVB & -0.02 & 0.00 & 0.00 & 0.00 & 0.02 & 0.04 & -0.01 & 0.00 & 0.02 & -0.02 & 0.02 \\
\hline Correl. Coeff. & dS & $\omega$ & Diversif & Body Size & Elev & Lat & Temp & HR & NPP & Prec & UVB \\
\hline dS & 1.00 & -0.03 & 0.23 & -0.20 & -0.29 & -0.07 & 0.56 & 0.09 & -0.06 & -0.03 & -0.25 \\
\hline$\omega$ & -0.03 & 1.00 & 0.01 & 0.08 & 0.00 & 0.02 & -0.01 & 0.04 & -0.01 & 0.04 & 0.00 \\
\hline Diversif & 0.23 & 0.01 & 1.00 & -0.11 & -0.05 & -0.12 & 0.11 & 0.02 & -0.03 & 0.05 & 0.04 \\
\hline Body Size & -0.20 & 0.08 & -0.11 & 1.00 & 0.11 & 0.08 & -0.09 & 0.09 & 0.04 & 0.06 & 0.06 \\
\hline Elev & -0.29 & 0.00 & -0.05 & 0.11 & 1.00 & 0.13 & -0.55 & -0.29 & -0.14 & -0.24 & 0.13 \\
\hline Lat & -0.07 & 0.02 & -0.12 & 0.08 & 0.13 & 1.00 & -0.14 & 0.00 & 0.19 & -0.18 & 0.14 \\
\hline Temp & 0.56 & -0.01 & 0.11 & -0.09 & -0.55 & -0.14 & 1.00 & 0.27 & 0.22 & 0.41 & -0.32 \\
\hline $\mathrm{HR}$ & 0.09 & 0.04 & 0.02 & 0.09 & -0.29 & 0.00 & 0.27 & 1.00 & -0.11 & 0.53 & -0.26 \\
\hline NPP & -0.06 & -0.01 & -0.03 & 0.04 & -0.14 & 0.19 & 0.22 & -0.11 & 1.00 & -0.04 & 0.21 \\
\hline Prec & -0.03 & 0.04 & 0.05 & 0.06 & -0.24 & -0.18 & 0.41 & 0.53 & -0.04 & 1.00 & -0.28 \\
\hline UVB & -0.25 & 0.00 & 0.04 & 0.06 & 0.13 & 0.14 & -0.32 & -0.26 & 0.21 & -0.28 & 1.00 \\
\hline Post. Prob. & dS & $\omega$ & Diversif & Body Size & Elev & Lat & Temp & HR & NPP & Prec & UVB \\
\hline $\mathrm{dS}$ & - & 0.47 & 0.97 & 0.08 & 0.02 & 0.33 & 1.00 & 0.72 & 0.36 & 0.41 & 0.05 \\
\hline$\omega$ & 0.47 & - & 0.51 & 0.62 & 0.50 & 0.54 & 0.50 & 0.55 & 0.49 & 0.54 & 0.50 \\
\hline Diversif & 0.97 & 0.51 & - & 0.15 & 0.31 & 0.13 & 0.85 & 0.56 & 0.38 & 0.69 & 0.64 \\
\hline Body Size & 0.08 & 0.62 & 0.15 & - & 0.85 & 0.77 & 0.18 & 0.80 & 0.65 & 0.71 & 0.72 \\
\hline Elev & 0.02 & 0.50 & 0.31 & 0.85 & - & 0.88 & 0.00 & 0.00 & 0.09 & 0.01 & 0.89 \\
\hline Lat & 0.33 & 0.54 & 0.13 & 0.77 & 0.88 & - & 0.09 & 0.51 & 0.97 & 0.04 & 0.91 \\
\hline Temp & 1.00 & 0.50 & 0.85 & 0.18 & 0.00 & 0.09 & - & 1.00 & 0.98 & 1.00 & 0.00 \\
\hline $\mathrm{HR}$ & 0.72 & 0.55 & 0.56 & 0.80 & 0.00 & 0.51 & 1.00 & - & 0.15 & 1.00 & 0.01 \\
\hline NPP & 0.36 & 0.49 & 0.38 & 0.65 & 0.09 & 0.97 & 0.98 & 0.15 & - & 0.34 & 0.98 \\
\hline Prec & 0.41 & 0.54 & 0.69 & 0.71 & 0.01 & 0.04 & 1.00 & 1.00 & 0.34 & - & 0.00 \\
\hline UVB & 0.05 & 0.50 & 0.64 & 0.72 & 0.89 & 0.91 & 0.00 & 0.01 & 0.98 & 0.00 & - \\
\hline
\end{tabular}




\begin{tabular}{|c|c|c|c|c|c|c|c|c|c|c|c|}
\hline Precisions & dS & $\omega$ & Diversif & Body Size & Elev & Lat & Temp & HR & NPP & Prec & UVB \\
\hline dS & 14.50 & 1.53 & -3.57 & 2.75 & -0.14 & -0.13 & -26.20 & -5.48 & 1.92 & 4.02 & 3.54 \\
\hline$\omega$ & 1.53 & 3560.00 & -3.18 & -19.90 & 0.17 & -0.36 & 3.02 & -16.30 & 1.79 & -2.69 & -1.32 \\
\hline Diversif & -3.57 & -3.18 & 16.60 & 1.27 & -0.10 & 0.24 & 3.86 & 1.16 & -0.14 & -1.21 & -4.64 \\
\hline Body Size & 2.75 & -19.90 & 1.27 & 33.10 & -0.64 & -0.12 & -2.94 & -8.60 & -0.18 & 0.08 & -2.02 \\
\hline Elev & -0.14 & 0.17 & -0.10 & -0.64 & 1.52 & -0.06 & 4.92 & 3.82 & 0.11 & -0.23 & 0.90 \\
\hline Lat & -0.13 & -0.36 & 0.24 & -0.12 & -0.06 & 0.40 & 0.51 & -1.26 & -0.26 & 0.19 & -0.28 \\
\hline Temp & -26.20 & 3.02 & 3.86 & -2.94 & 4.92 & 0.51 & 138.00 & 14.50 & -8.84 & -13.70 & 15.70 \\
\hline $\mathrm{HR}$ & -5.48 & -16.30 & 1.16 & -8.60 & 3.82 & -1.26 & 14.50 & 234.00 & 2.90 & -16.40 & 12.70 \\
\hline NPP & 1.92 & 1.79 & -0.14 & -0.18 & 0.11 & -0.26 & -8.84 & 2.90 & 4.54 & 0.69 & -3.87 \\
\hline Prec & 4.02 & -2.69 & -1.21 & 0.08 & -0.23 & 0.19 & -13.70 & -16.40 & 0.69 & 7.25 & 2.75 \\
\hline UVB & 3.54 & -1.32 & -4.64 & -2.02 & 0.90 & -0.28 & 15.70 & 12.70 & -3.87 & 2.75 & 94.50 \\
\hline Partial C.C. & dS & $\omega$ & Diversif & Body Size & Elev & Lat & Temp & HR & NPP & Prec & UVB \\
\hline dS & -1.00 & -0.01 & 0.23 & -0.12 & 0.03 & 0.05 & 0.58 & 0.09 & -0.24 & -0.39 & -0.09 \\
\hline$\omega$ & -0.01 & -1.00 & 0.02 & 0.10 & 0.00 & 0.03 & -0.01 & 0.03 & -0.02 & 0.03 & 0.01 \\
\hline Diversif & 0.23 & 0.02 & -1.00 & -0.06 & 0.02 & -0.09 & -0.07 & -0.02 & 0.01 & 0.11 & 0.12 \\
\hline Body Size & -0.12 & 0.10 & -0.06 & -1.00 & 0.09 & 0.03 & 0.04 & 0.10 & 0.02 & 0.00 & 0.04 \\
\hline Elev & 0.03 & 0.00 & 0.02 & 0.09 & -1.00 & 0.08 & -0.35 & -0.21 & -0.04 & 0.07 & -0.08 \\
\hline Lat & 0.05 & 0.03 & -0.09 & 0.03 & 0.08 & -1.00 & -0.07 & 0.14 & 0.19 & -0.12 & 0.05 \\
\hline Temp & 0.58 & -0.01 & -0.07 & 0.04 & -0.35 & -0.07 & -1.00 & -0.08 & 0.35 & 0.43 & -0.14 \\
\hline $\mathrm{HR}$ & 0.09 & 0.03 & -0.02 & 0.10 & -0.21 & 0.14 & -0.08 & -1.00 & -0.09 & 0.41 & -0.09 \\
\hline NPP & -0.24 & -0.02 & 0.01 & 0.02 & -0.04 & 0.19 & 0.35 & -0.09 & -1.00 & -0.12 & 0.19 \\
\hline Prec & -0.39 & 0.03 & 0.11 & 0.00 & 0.07 & -0.12 & 0.43 & 0.41 & -0.12 & -1.00 & -0.10 \\
\hline UVB & -0.09 & 0.01 & 0.12 & 0.04 & -0.08 & 0.05 & -0.14 & -0.09 & 0.19 & -0.10 & -1.00 \\
\hline Post. Prob. & dS & $\omega$ & Diversif & Body Size & Elev & Lat & Temp & HR & NPP & Prec & UVB \\
\hline dS & - & 0.50 & 0.92 & 0.24 & 0.56 & 0.60 & 1.00 & 0.69 & 0.12 & 0.02 & 0.31 \\
\hline$\omega$ & 0.50 & - & 0.53 & 0.62 & 0.50 & 0.54 & 0.49 & 0.54 & 0.48 & 0.53 & 0.51 \\
\hline Diversif & 0.92 & 0.53 & - & 0.33 & 0.56 & 0.24 & 0.30 & 0.45 & 0.53 & 0.79 & 0.82 \\
\hline Body Size & 0.24 & 0.62 & 0.33 & - & 0.76 & 0.61 & 0.60 & 0.77 & 0.55 & 0.50 & 0.62 \\
\hline Elev & 0.56 & 0.50 & 0.56 & 0.76 & - & 0.74 & 0.02 & 0.07 & 0.38 & 0.69 & 0.27 \\
\hline Lat & 0.60 & 0.54 & 0.24 & 0.61 & 0.74 & - & 0.34 & 0.84 & 0.91 & 0.21 & 0.64 \\
\hline Temp & 1.00 & 0.49 & 0.30 & 0.60 & 0.02 & 0.34 & - & 0.30 & 0.98 & 0.99 & 0.18 \\
\hline $\mathrm{HR}$ & 0.69 & 0.54 & 0.45 & 0.77 & 0.07 & 0.84 & 0.30 & - & 0.25 & 0.99 & 0.25 \\
\hline NPP & 0.12 & 0.48 & 0.53 & 0.55 & 0.38 & 0.91 & 0.98 & 0.25 & - & 0.22 & 0.91 \\
\hline Prec & 0.02 & 0.53 & 0.79 & 0.50 & 0.69 & 0.21 & 0.99 & 0.99 & 0.22 & - & 0.23 \\
\hline UVB & 0.31 & 0.51 & 0.82 & 0.62 & 0.27 & 0.64 & 0.18 & 0.25 & 0.91 & 0.23 & - \\
\hline
\end{tabular}




\begin{tabular}{|c|c|c|c|c|c|c|c|c|c|c|}
\hline \multicolumn{11}{|c|}{ MITOCHONDRIAL CODING-GENE: $\omega$ ANALYSIS 2 (Controlling for Temperature) } \\
\hline Covariances & dS & $\omega$ & Diversif & Body Size & Elev & Lat & HR & NPP & Prec & UVB \\
\hline dS & 0.18 & 0.00 & 0.03 & -0.02 & 0.01 & 0.01 & 0.00 & -0.06 & -0.08 & 0.00 \\
\hline$\omega$ & 0.00 & 0.04 & 0.00 & 0.00 & 0.00 & 0.02 & 0.00 & 0.00 & 0.00 & 0.00 \\
\hline Diversif & 0.03 & 0.00 & 0.08 & -0.01 & 0.00 & -0.06 & 0.00 & -0.01 & 0.00 & 0.00 \\
\hline Body Size & -0.02 & 0.00 & -0.01 & 0.04 & 0.01 & 0.03 & 0.00 & 0.01 & 0.01 & 0.00 \\
\hline Elev & 0.01 & 0.00 & 0.00 & 0.01 & 0.92 & 0.10 & -0.02 & -0.01 & -0.01 & -0.01 \\
\hline Lat & 0.01 & 0.02 & -0.06 & 0.03 & 0.10 & 3.72 & 0.01 & 0.27 & -0.15 & 0.03 \\
\hline $\mathrm{HR}$ & 0.00 & 0.00 & 0.00 & 0.00 & -0.02 & 0.01 & 0.01 & -0.01 & 0.02 & 0.00 \\
\hline NPP & -0.06 & 0.00 & -0.01 & 0.01 & -0.01 & 0.27 & -0.01 & 0.38 & -0.05 & 0.02 \\
\hline Prec & -0.08 & 0.00 & 0.00 & 0.01 & -0.01 & -0.15 & 0.02 & -0.05 & 0.31 & -0.01 \\
\hline UVB & 0.00 & 0.00 & 0.00 & 0.00 & -0.01 & 0.03 & 0.00 & 0.02 & -0.01 & 0.02 \\
\hline Correl. Coeff. & dS & $\omega$ & Diversif & Body Size & Elev & Lat & HR & NPP & Prec & UVB \\
\hline $\mathrm{dS}$ & 1.00 & -0.03 & 0.21 & -0.18 & 0.03 & 0.02 & -0.07 & -0.23 & -0.36 & -0.08 \\
\hline$\omega$ & -0.03 & 1.00 & 0.01 & 0.08 & 0.00 & 0.03 & 0.05 & -0.01 & 0.04 & 0.00 \\
\hline Diversif & 0.21 & 0.01 & 1.00 & -0.10 & 0.01 & -0.10 & -0.01 & -0.06 & 0.01 & 0.08 \\
\hline Body Size & -0.18 & 0.08 & -0.10 & 1.00 & 0.07 & 0.07 & 0.12 & 0.06 & 0.11 & 0.04 \\
\hline Elev & 0.03 & 0.00 & 0.01 & 0.07 & 1.00 & 0.06 & -0.18 & -0.02 & -0.02 & -0.06 \\
\hline Lat & 0.02 & 0.03 & -0.10 & 0.07 & 0.06 & 1.00 & 0.04 & 0.23 & -0.14 & 0.10 \\
\hline $\mathrm{HR}$ & -0.07 & 0.05 & -0.01 & 0.12 & -0.18 & 0.04 & 1.00 & -0.18 & 0.47 & -0.19 \\
\hline NPP & -0.23 & -0.01 & -0.06 & 0.06 & -0.02 & 0.23 & -0.18 & 1.00 & -0.15 & 0.30 \\
\hline Prec & -0.36 & 0.04 & 0.01 & 0.11 & -0.02 & -0.14 & 0.47 & -0.15 & 1.00 & -0.18 \\
\hline UVB & -0.08 & 0.00 & 0.08 & 0.04 & -0.06 & 0.10 & -0.19 & 0.30 & -0.18 & 1.00 \\
\hline Post. Prob. & dS & $\omega$ & Diversif & Body Size & Elev & Lat & HR & NPP & Prec & UVB \\
\hline $\mathrm{dS}$ & - & 0.46 & 0.95 & 0.11 & 0.57 & 0.53 & 0.34 & 0.11 & 0.02 & 0.30 \\
\hline$\omega$ & 0.46 & - & 0.51 & 0.62 & 0.49 & 0.54 & 0.56 & 0.49 & 0.55 & 0.50 \\
\hline Diversif & 0.95 & 0.51 & - & 0.17 & 0.54 & 0.17 & 0.45 & 0.29 & 0.53 & 0.77 \\
\hline Body Size & 0.11 & 0.62 & 0.17 & - & 0.74 & 0.74 & 0.87 & 0.73 & 0.84 & 0.63 \\
\hline Elev & 0.57 & 0.49 & 0.54 & 0.74 & - & 0.70 & 0.04 & 0.41 & 0.41 & 0.27 \\
\hline Lat & 0.53 & 0.54 & 0.17 & 0.74 & 0.70 & - & 0.65 & 0.99 & 0.09 & 0.83 \\
\hline$H R$ & 0.34 & 0.56 & 0.45 & 0.87 & 0.04 & 0.65 & - & 0.04 & 1.00 & 0.04 \\
\hline NPP & 0.11 & 0.49 & 0.29 & 0.73 & 0.41 & 0.99 & 0.04 & - & 0.07 & 1.00 \\
\hline Prec & 0.02 & 0.55 & 0.53 & 0.84 & 0.41 & 0.09 & 1.00 & 0.07 & - & 0.05 \\
\hline UVB & 0.30 & 0.50 & 0.77 & 0.63 & 0.27 & 0.83 & 0.04 & 1.00 & 0.05 & - \\
\hline
\end{tabular}




\begin{tabular}{|c|c|c|c|c|c|c|c|c|c|c|c|}
\hline \multicolumn{12}{|c|}{ NUCLEAR CODING-GENES: dN ANALYSIS 1} \\
\hline Covariances & dS & $d N$ & Diversif & Body Size & Elev & Lat & Temp & HR & NPP & Prec & UVB \\
\hline dS & 0.13 & 0.05 & 0.01 & -0.01 & -0.21 & -0.11 & 0.04 & 0.01 & 0.06 & 0.06 & -0.01 \\
\hline $\mathrm{dN}$ & 0.05 & 0.22 & 0.01 & -0.01 & 0.02 & -0.09 & 0.00 & 0.00 & 0.01 & -0.06 & 0.01 \\
\hline Diversif & 0.01 & 0.01 & 0.10 & 0.00 & -0.02 & -0.05 & 0.01 & 0.00 & 0.00 & 0.00 & 0.00 \\
\hline Body Size & -0.01 & -0.01 & 0.00 & 0.06 & 0.04 & 0.06 & 0.00 & 0.01 & -0.01 & 0.02 & 0.00 \\
\hline Elev & -0.21 & 0.02 & -0.02 & 0.04 & 1.68 & 0.39 & -0.13 & -0.04 & -0.10 & -0.17 & 0.03 \\
\hline Lat & -0.11 & -0.09 & -0.05 & 0.06 & 0.39 & 5.00 & -0.05 & 0.01 & 0.13 & -0.20 & 0.08 \\
\hline Temp & 0.04 & 0.00 & 0.01 & 0.00 & -0.13 & -0.05 & 0.04 & 0.01 & 0.04 & 0.05 & -0.01 \\
\hline$H R$ & 0.01 & 0.00 & 0.00 & 0.01 & -0.04 & 0.01 & 0.01 & 0.01 & 0.00 & 0.04 & 0.00 \\
\hline NPP & 0.06 & 0.01 & 0.00 & -0.01 & -0.10 & 0.13 & 0.04 & 0.00 & 0.31 & 0.06 & 0.02 \\
\hline Prec & 0.06 & -0.06 & 0.00 & 0.02 & -0.17 & -0.20 & 0.05 & 0.04 & 0.06 & 0.47 & -0.03 \\
\hline UVB & -0.01 & 0.01 & 0.00 & 0.00 & 0.03 & 0.08 & -0.01 & 0.00 & 0.02 & -0.03 & 0.03 \\
\hline Correl. Coeff. & dS & $d N$ & Diversif & Body Size & Elev & Lat & Temp & HR & NPP & Prec & UVB \\
\hline dS & 1.00 & 0.12 & 0.04 & -0.14 & -0.49 & -0.15 & 0.59 & 0.27 & 0.31 & 0.26 & -0.19 \\
\hline$d N$ & 0.12 & 1.00 & 0.02 & -0.12 & 0.04 & -0.06 & -0.07 & -0.11 & 0.00 & -0.21 & 0.06 \\
\hline Diversif & 0.04 & 0.02 & 1.00 & -0.06 & -0.04 & -0.07 & 0.10 & 0.00 & 0.01 & 0.00 & 0.07 \\
\hline Body Size & -0.14 & -0.12 & -0.06 & 1.00 & 0.12 & 0.10 & -0.11 & 0.17 & -0.05 & 0.10 & 0.06 \\
\hline Elev & -0.49 & 0.04 & -0.04 & 0.12 & 1.00 & 0.13 & -0.52 & -0.28 & -0.14 & -0.20 & 0.15 \\
\hline Lat & -0.15 & -0.06 & -0.07 & 0.10 & 0.13 & 1.00 & -0.13 & 0.02 & 0.10 & -0.13 & 0.22 \\
\hline Temp & 0.59 & -0.07 & 0.10 & -0.11 & -0.52 & -0.13 & 1.00 & 0.22 & 0.33 & 0.38 & -0.29 \\
\hline $\mathrm{HR}$ & 0.27 & -0.11 & 0.00 & 0.17 & -0.28 & 0.02 & 0.22 & 1.00 & 0.04 & 0.53 & -0.23 \\
\hline NPP & 0.31 & 0.00 & 0.01 & -0.05 & -0.14 & 0.10 & 0.33 & 0.04 & 1.00 & 0.15 & 0.19 \\
\hline Prec & 0.26 & -0.21 & 0.00 & 0.10 & -0.20 & -0.13 & 0.38 & 0.53 & 0.15 & 1.00 & -0.29 \\
\hline UVB & -0.19 & 0.06 & 0.07 & 0.06 & 0.15 & 0.22 & -0.29 & -0.23 & 0.19 & -0.29 & 1.00 \\
\hline Post. Prob. & dS & $d N$ & Diversif & Body Size & Elev & Lat & Temp & HR & NPP & Prec & UVB \\
\hline dS & - & 0.64 & 0.60 & 0.25 & 0.01 & 0.25 & 1.00 & 0.87 & 0.92 & 0.86 & 0.19 \\
\hline$d N$ & 0.64 & - & 0.56 & 0.30 & 0.55 & 0.41 & 0.40 & 0.35 & 0.49 & 0.23 & 0.59 \\
\hline Diversif & 0.60 & 0.56 & - & 0.30 & 0.34 & 0.26 & 0.83 & 0.50 & 0.55 & 0.50 & 0.73 \\
\hline Body Size & 0.25 & 0.30 & 0.30 & - & 0.86 & 0.82 & 0.17 & 0.94 & 0.32 & 0.82 & 0.71 \\
\hline Elev & 0.01 & 0.55 & 0.34 & 0.86 & - & 0.89 & 0.00 & 0.01 & 0.11 & 0.04 & 0.91 \\
\hline Lat & 0.25 & 0.41 & 0.26 & 0.82 & 0.89 & - & 0.12 & 0.57 & 0.83 & 0.12 & 0.98 \\
\hline Temp & 1.00 & 0.40 & 0.83 & 0.17 & 0.00 & 0.12 & - & 0.98 & 1.00 & 1.00 & 0.00 \\
\hline $\mathrm{HR}$ & 0.87 & 0.35 & 0.50 & 0.94 & 0.01 & 0.57 & 0.98 & - & 0.65 & 1.00 & 0.02 \\
\hline NPP & 0.92 & 0.49 & 0.55 & 0.32 & 0.11 & 0.83 & 1.00 & 0.65 & - & 0.91 & 0.96 \\
\hline Prec & 0.86 & 0.23 & 0.50 & 0.82 & 0.04 & 0.12 & 1.00 & 1.00 & 0.91 & - & 0.00 \\
\hline UVB & 0.19 & 0.59 & 0.73 & 0.71 & 0.91 & 0.98 & 0.00 & 0.02 & 0.96 & 0.00 & - \\
\hline
\end{tabular}




\begin{tabular}{|c|c|c|c|c|c|c|c|c|c|c|c|}
\hline Precisions & dS & $\mathrm{dN}$ & Diversif & Body Size & Elev & Lat & Temp & HR & NPP & Prec & UVB \\
\hline $\mathrm{dS}$ & 63.40 & -1.06 & 1.56 & 3.68 & 3.07 & 0.38 & -34.90 & -18.40 & -4.89 & -0.68 & 3.68 \\
\hline $\mathrm{dN}$ & -1.06 & 1390.00 & -0.11 & 4.83 & -0.63 & 0.34 & 12.30 & 2.71 & 0.02 & 4.92 & -6.32 \\
\hline Diversif & 1.56 & -0.11 & 13.20 & 1.00 & -0.02 & 0.15 & -4.14 & -1.18 & 0.28 & 0.13 & -3.38 \\
\hline Body Size & 3.68 & 4.83 & 1.00 & 25.70 & -0.39 & -0.08 & 0.01 & -10.30 & 0.25 & -0.31 & -3.02 \\
\hline Elev & 3.07 & -0.63 & -0.02 & -0.39 & 1.43 & -0.04 & 2.09 & 2.33 & -0.28 & -0.26 & 0.76 \\
\hline Lat & 0.38 & 0.34 & 0.15 & -0.08 & -0.04 & 0.33 & -0.21 & -1.03 & -0.17 & 0.21 & -0.56 \\
\hline Temp & -34.90 & 12.30 & -4.14 & 0.01 & 2.09 & -0.21 & 92.00 & 17.60 & -3.72 & -3.77 & 15.90 \\
\hline $\mathrm{HR}$ & -18.40 & 2.71 & -1.18 & -10.30 & 2.33 & -1.03 & 17.60 & 180.00 & 2.48 & -12.40 & 9.67 \\
\hline NPP & -4.89 & 0.02 & 0.28 & 0.25 & -0.28 & -0.17 & -3.72 & 2.48 & 6.56 & -0.55 & -6.08 \\
\hline Prec & -0.68 & 4.92 & 0.13 & -0.31 & -0.26 & 0.21 & -3.77 & -12.40 & -0.55 & 5.41 & 2.33 \\
\hline UVB & 3.68 & -6.32 & -3.38 & -3.02 & 0.76 & -0.56 & 15.90 & 9.67 & -6.08 & 2.33 & 76.20 \\
\hline Partial C.C. & dS & $d N$ & Diversif & Body Size & Elev & Lat & Temp & HR & NPP & Prec & UVB \\
\hline dS & -1.00 & 0.20 & -0.05 & -0.08 & -0.30 & -0.09 & 0.43 & 0.17 & 0.22 & 0.03 & -0.05 \\
\hline$d N$ & 0.20 & -1.00 & 0.02 & -0.10 & 0.09 & -0.09 & -0.11 & 0.00 & 0.01 & -0.21 & 0.03 \\
\hline Diversif & -0.05 & 0.02 & -1.00 & -0.05 & 0.01 & -0.07 & 0.12 & 0.03 & -0.03 & -0.02 & 0.11 \\
\hline Body Size & -0.08 & -0.10 & -0.05 & -1.00 & 0.07 & 0.03 & -0.01 & 0.15 & -0.03 & 0.03 & 0.07 \\
\hline Elev & -0.30 & 0.09 & 0.01 & 0.07 & -1.00 & 0.06 & -0.21 & -0.16 & 0.09 & 0.10 & -0.07 \\
\hline Lat & -0.09 & -0.09 & -0.07 & 0.03 & 0.06 & -1.00 & 0.03 & 0.14 & 0.11 & -0.16 & 0.12 \\
\hline Temp & 0.43 & -0.11 & 0.12 & -0.01 & -0.21 & 0.03 & -1.00 & -0.13 & 0.18 & 0.18 & -0.20 \\
\hline $\mathrm{HR}$ & 0.17 & 0.00 & 0.03 & 0.15 & -0.16 & 0.14 & -0.13 & -1.00 & -0.06 & 0.41 & -0.09 \\
\hline NPP & 0.22 & 0.01 & -0.03 & -0.03 & 0.09 & 0.11 & 0.18 & -0.06 & -1.00 & 0.09 & 0.28 \\
\hline Prec & 0.03 & -0.21 & -0.02 & 0.03 & 0.10 & -0.16 & 0.18 & 0.41 & 0.09 & -1.00 & -0.12 \\
\hline UVB & -0.05 & 0.03 & 0.11 & 0.07 & -0.07 & 0.12 & -0.20 & -0.09 & 0.28 & -0.12 & -1.00 \\
\hline Post. Prob. & dS & $d N$ & Diversif & Body Size & Elev & Lat & Temp & HR & NPP & Prec & UVB \\
\hline dS & - & 0.73 & 0.40 & 0.39 & 0.13 & 0.38 & 0.95 & 0.73 & 0.77 & 0.54 & 0.43 \\
\hline$d N$ & 0.73 & - & 0.55 & 0.36 & 0.63 & 0.38 & 0.34 & 0.50 & 0.51 & 0.23 & 0.54 \\
\hline Diversif & 0.40 & 0.55 & - & 0.34 & 0.52 & 0.29 & 0.80 & 0.58 & 0.39 & 0.45 & 0.81 \\
\hline Body Size & 0.39 & 0.36 & 0.34 & - & 0.68 & 0.60 & 0.48 & 0.85 & 0.43 & 0.60 & 0.69 \\
\hline Elev & 0.13 & 0.63 & 0.52 & 0.68 & - & 0.65 & 0.15 & 0.17 & 0.70 & 0.72 & 0.32 \\
\hline Lat & 0.38 & 0.38 & 0.29 & 0.60 & 0.65 & - & 0.57 & 0.82 & 0.77 & 0.16 & 0.79 \\
\hline Temp & 0.95 & 0.34 & 0.80 & 0.48 & 0.15 & 0.57 & - & 0.23 & 0.81 & 0.84 & 0.13 \\
\hline $\mathrm{HR}$ & 0.73 & 0.50 & 0.58 & 0.85 & 0.17 & 0.82 & 0.23 & - & 0.35 & 0.98 & 0.27 \\
\hline NPP & 0.77 & 0.51 & 0.39 & 0.43 & 0.70 & 0.77 & 0.81 & 0.35 & - & 0.73 & 0.96 \\
\hline Prec & 0.54 & 0.23 & 0.45 & 0.60 & 0.72 & 0.16 & 0.84 & 0.98 & 0.73 & - & 0.21 \\
\hline UVB & 0.43 & 0.54 & 0.81 & 0.69 & 0.32 & 0.79 & 0.13 & 0.27 & 0.96 & 0.21 & - \\
\hline
\end{tabular}




\begin{tabular}{|c|c|c|c|c|c|c|c|c|c|c|}
\hline \multicolumn{11}{|c|}{ NUCLEAR CODING-GENES: dN ANALYSIS 1 (Controlling for Temperature) } \\
\hline Covariances & dS & $d \mathbf{N}$ & Diversif & Body Size & Elev & Lat & HR & NPP & Prec & UVB \\
\hline dS & 0.08 & 0.04 & 0.00 & -0.01 & -0.08 & -0.06 & 0.01 & 0.02 & 0.01 & 0.00 \\
\hline $\mathrm{dN}$ & 0.04 & 0.20 & 0.01 & -0.02 & 0.00 & -0.10 & 0.00 & 0.01 & -0.06 & 0.00 \\
\hline Diversif & 0.00 & 0.01 & 0.10 & 0.00 & 0.00 & -0.04 & 0.00 & 0.00 & -0.01 & 0.00 \\
\hline Body Size & -0.01 & -0.02 & 0.00 & 0.06 & 0.02 & 0.05 & 0.01 & 0.00 & 0.02 & 0.00 \\
\hline Elev & -0.08 & 0.00 & 0.00 & 0.02 & 1.21 & 0.19 & -0.02 & 0.03 & 0.00 & 0.00 \\
\hline Lat & -0.06 & -0.10 & -0.04 & 0.05 & 0.19 & 4.86 & 0.01 & 0.18 & -0.13 & 0.06 \\
\hline $\mathrm{HR}$ & 0.01 & 0.00 & 0.00 & 0.01 & -0.02 & 0.01 & 0.01 & 0.00 & 0.03 & 0.00 \\
\hline NPP & 0.02 & 0.01 & 0.00 & 0.00 & 0.03 & 0.18 & 0.00 & 0.27 & 0.01 & 0.03 \\
\hline Prec & 0.01 & -0.06 & -0.01 & 0.02 & 0.00 & -0.13 & 0.03 & 0.01 & 0.40 & -0.02 \\
\hline UVB & 0.00 & 0.00 & 0.00 & 0.00 & 0.00 & 0.06 & 0.00 & 0.03 & -0.02 & 0.02 \\
\hline Correl. Coeff. & dS & $d N$ & Diversif & Body Size & Elev & Lat & HR & NPP & Prec & UVB \\
\hline $\mathrm{dS}$ & 1.00 & 0.20 & -0.03 & -0.09 & -0.28 & -0.09 & 0.18 & 0.15 & 0.05 & -0.02 \\
\hline $\mathrm{dN}$ & 0.20 & 1.00 & 0.03 & -0.14 & 0.01 & -0.08 & -0.10 & 0.03 & -0.21 & 0.05 \\
\hline Diversif & -0.03 & 0.03 & 1.00 & -0.05 & 0.01 & -0.06 & -0.02 & -0.02 & -0.04 & 0.10 \\
\hline Body Size & -0.09 & -0.14 & -0.05 & 1.00 & 0.07 & 0.09 & 0.20 & -0.02 & 0.16 & 0.03 \\
\hline Elev & -0.28 & 0.01 & 0.01 & 0.07 & 1.00 & 0.08 & -0.20 & 0.04 & 0.00 & 0.00 \\
\hline Lat & -0.09 & -0.08 & -0.06 & 0.09 & 0.08 & 1.00 & 0.05 & 0.15 & -0.09 & 0.19 \\
\hline$H R$ & 0.18 & -0.10 & -0.02 & 0.20 & -0.20 & 0.05 & 1.00 & -0.03 & 0.50 & -0.17 \\
\hline NPP & 0.15 & 0.03 & -0.02 & -0.02 & 0.04 & 0.15 & -0.03 & 1.00 & 0.03 & 0.32 \\
\hline Prec & 0.05 & -0.21 & -0.04 & 0.16 & 0.00 & -0.09 & 0.50 & 0.03 & 1.00 & -0.20 \\
\hline UVB & -0.02 & 0.05 & 0.10 & 0.03 & 0.00 & 0.19 & -0.17 & 0.32 & -0.20 & 1.00 \\
\hline Post. Prob. & dS & $d N$ & Diversif & Body Size & Elev & Lat & HR & NPP & Prec & UVB \\
\hline $\mathrm{dS}$ & - & 0.71 & 0.43 & 0.36 & 0.12 & 0.36 & 0.73 & 0.70 & 0.57 & 0.47 \\
\hline $\mathrm{dN}$ & 0.71 & - & 0.58 & 0.29 & 0.51 & 0.39 & 0.36 & 0.54 & 0.24 & 0.57 \\
\hline Diversif & 0.43 & 0.58 & - & 0.33 & 0.54 & 0.30 & 0.42 & 0.42 & 0.35 & 0.83 \\
\hline Body Size & 0.36 & 0.29 & 0.33 & - & 0.74 & 0.79 & 0.96 & 0.44 & 0.92 & 0.63 \\
\hline Elev & 0.12 & 0.51 & 0.54 & 0.74 & - & 0.77 & 0.04 & 0.66 & 0.49 & 0.48 \\
\hline Lat & 0.36 & 0.39 & 0.30 & 0.79 & 0.77 & - & 0.68 & 0.92 & 0.21 & 0.96 \\
\hline$H R$ & 0.73 & 0.36 & 0.42 & 0.96 & 0.04 & 0.68 & - & 0.38 & 1.00 & 0.06 \\
\hline NPP & 0.70 & 0.54 & 0.42 & 0.44 & 0.66 & 0.92 & 0.38 & - & 0.60 & 1.00 \\
\hline Prec & 0.57 & 0.24 & 0.35 & 0.92 & 0.49 & 0.21 & 1.00 & 0.60 & - & 0.03 \\
\hline UVB & 0.47 & 0.57 & 0.83 & 0.63 & 0.48 & 0.96 & 0.06 & 1.00 & 0.03 & - \\
\hline
\end{tabular}




\begin{tabular}{|c|c|c|c|c|c|c|c|c|c|c|c|}
\hline \multicolumn{12}{|c|}{ NUCLEAR CODING-GENES: dN ANALYSIS 2} \\
\hline Covariances & dS & $d N$ & Diversif & Body Size & Elev & Lat & Temp & HR & NPP & Prec & UVB \\
\hline dS & 0.13 & 0.04 & 0.00 & -0.01 & -0.22 & -0.12 & 0.04 & 0.01 & 0.06 & 0.06 & -0.01 \\
\hline $\mathrm{dN}$ & 0.04 & 0.22 & 0.01 & -0.01 & 0.01 & -0.10 & 0.00 & 0.00 & 0.00 & -0.07 & 0.00 \\
\hline Diversif & 0.00 & 0.01 & 0.10 & 0.00 & -0.02 & -0.05 & 0.01 & 0.00 & 0.00 & 0.00 & 0.00 \\
\hline Body Size & -0.01 & -0.01 & 0.00 & 0.06 & 0.04 & 0.06 & -0.01 & 0.01 & -0.01 & 0.02 & 0.00 \\
\hline Elev & -0.22 & 0.01 & -0.02 & 0.04 & 1.68 & 0.39 & -0.13 & -0.04 & -0.10 & -0.18 & 0.03 \\
\hline Lat & -0.12 & -0.10 & -0.05 & 0.06 & 0.39 & 5.01 & -0.05 & 0.01 & 0.13 & -0.21 & 0.08 \\
\hline Temp & 0.04 & 0.00 & 0.01 & -0.01 & -0.13 & -0.05 & 0.04 & 0.01 & 0.04 & 0.05 & -0.01 \\
\hline HR & 0.01 & 0.00 & 0.00 & 0.01 & -0.04 & 0.01 & 0.01 & 0.01 & 0.00 & 0.04 & 0.00 \\
\hline NPP & 0.06 & 0.00 & 0.00 & -0.01 & -0.10 & 0.13 & 0.04 & 0.00 & 0.31 & 0.06 & 0.02 \\
\hline Prec & 0.06 & -0.07 & 0.00 & 0.02 & -0.18 & -0.21 & 0.05 & 0.04 & 0.06 & 0.47 & -0.03 \\
\hline UVB & -0.01 & 0.00 & 0.00 & 0.00 & 0.03 & 0.08 & -0.01 & 0.00 & 0.02 & -0.03 & 0.03 \\
\hline Correl. Coeff. & dS & $d N$ & Diversif & Body Size & Elev & Lat & Temp & HR & NPP & Prec & UVB \\
\hline dS & 1.00 & 0.13 & 0.04 & -0.14 & -0.50 & -0.15 & 0.59 & 0.27 & 0.31 & 0.26 & -0.19 \\
\hline$d N$ & 0.13 & 1.00 & 0.03 & -0.12 & 0.04 & -0.07 & -0.06 & -0.10 & 0.01 & -0.20 & 0.05 \\
\hline Diversif & 0.04 & 0.03 & 1.00 & -0.06 & -0.04 & -0.07 & 0.10 & 0.00 & 0.01 & 0.00 & 0.07 \\
\hline Body Size & -0.14 & -0.12 & -0.06 & 1.00 & 0.11 & 0.10 & -0.11 & 0.17 & -0.05 & 0.10 & 0.06 \\
\hline Elev & -0.50 & 0.04 & -0.04 & 0.11 & 1.00 & 0.13 & -0.52 & -0.29 & -0.14 & -0.20 & 0.15 \\
\hline Lat & -0.15 & -0.07 & -0.07 & 0.10 & 0.13 & 1.00 & -0.13 & 0.02 & 0.10 & -0.14 & 0.22 \\
\hline Temp & 0.59 & -0.06 & 0.10 & -0.11 & -0.52 & -0.13 & 1.00 & 0.23 & 0.33 & 0.38 & -0.29 \\
\hline$H R$ & 0.27 & -0.10 & 0.00 & 0.17 & -0.29 & 0.02 & 0.23 & 1.00 & 0.04 & 0.53 & -0.22 \\
\hline NPP & 0.31 & 0.01 & 0.01 & -0.05 & -0.14 & 0.10 & 0.33 & 0.04 & 1.00 & 0.15 & 0.20 \\
\hline Prec & 0.26 & -0.20 & 0.00 & 0.10 & -0.20 & -0.14 & 0.38 & 0.53 & 0.15 & 1.00 & -0.29 \\
\hline UVB & -0.19 & 0.05 & 0.07 & 0.06 & 0.15 & 0.22 & -0.29 & -0.22 & 0.20 & -0.29 & 1.00 \\
\hline Post. Prob. & dS & $d N$ & Diversif & Body Size & Elev & Lat & Temp & HR & NPP & Prec & UVB \\
\hline dS & - & 0.66 & 0.60 & 0.25 & 0.01 & 0.24 & 1.00 & 0.87 & 0.91 & 0.86 & 0.19 \\
\hline$d N$ & 0.66 & - & 0.56 & 0.30 & 0.55 & 0.41 & 0.42 & 0.36 & 0.51 & 0.24 & 0.57 \\
\hline Diversif & 0.60 & 0.56 & - & 0.30 & 0.36 & 0.26 & 0.83 & 0.50 & 0.54 & 0.51 & 0.73 \\
\hline Body Size & 0.25 & 0.30 & 0.30 & - & 0.85 & 0.82 & 0.16 & 0.94 & 0.33 & 0.82 & 0.72 \\
\hline Elev & 0.01 & 0.55 & 0.36 & 0.85 & - & 0.88 & 0.00 & 0.00 & 0.11 & 0.04 & 0.90 \\
\hline Lat & 0.24 & 0.41 & 0.26 & 0.82 & 0.88 & - & 0.12 & 0.58 & 0.82 & 0.11 & 0.97 \\
\hline Temp & 1.00 & 0.42 & 0.83 & 0.16 & 0.00 & 0.12 & - & 0.98 & 1.00 & 1.00 & 0.01 \\
\hline $\mathrm{HR}$ & 0.87 & 0.36 & 0.50 & 0.94 & 0.00 & 0.58 & 0.98 & - & 0.64 & 1.00 & 0.02 \\
\hline NPP & 0.91 & 0.51 & 0.54 & 0.33 & 0.11 & 0.82 & 1.00 & 0.64 & - & 0.91 & 0.96 \\
\hline Prec & 0.86 & 0.24 & 0.51 & 0.82 & 0.04 & 0.11 & 1.00 & 1.00 & 0.91 & - & 0.00 \\
\hline UVB & 0.19 & 0.57 & 0.73 & 0.72 & 0.90 & 0.97 & 0.01 & 0.02 & 0.96 & 0.00 & - \\
\hline
\end{tabular}




\begin{tabular}{|c|c|c|c|c|c|c|c|c|c|c|c|}
\hline Precisions & dS & $d N$ & Diversif & Body Size & Elev & Lat & Temp & HR & NPP & Prec & UVB \\
\hline dS & 60.00 & -4.10 & 1.40 & 3.60 & 3.01 & 0.39 & -34.50 & -17.80 & -4.74 & -0.70 & 3.97 \\
\hline $\mathrm{dN}$ & -4.10 & 1520.00 & -1.34 & 4.99 & -0.71 & 0.23 & 9.32 & 4.63 & -1.17 & 3.78 & -1.95 \\
\hline Diversif & 1.40 & -1.34 & 13.20 & 0.98 & -0.03 & 0.15 & -4.03 & -1.10 & 0.30 & 0.09 & -3.36 \\
\hline Body Size & 3.60 & 4.99 & 0.98 & 25.30 & -0.37 & -0.07 & -0.10 & -10.30 & 0.25 & -0.27 & -2.90 \\
\hline Elev & 3.01 & -0.71 & -0.03 & -0.37 & 1.42 & -0.04 & 2.05 & 2.40 & -0.28 & -0.27 & 0.73 \\
\hline Lat & 0.39 & 0.23 & 0.15 & -0.07 & -0.04 & 0.33 & -0.23 & -1.04 & -0.16 & 0.21 & -0.53 \\
\hline Temp & -34.50 & 9.32 & -4.03 & -0.10 & 2.05 & -0.23 & 91.60 & 17.50 & -3.61 & -3.83 & 15.80 \\
\hline$H R$ & -17.80 & 4.63 & -1.10 & -10.30 & 2.40 & -1.04 & 17.50 & 179.00 & 2.67 & -12.40 & 9.99 \\
\hline NPP & -4.74 & -1.17 & 0.30 & 0.25 & -0.28 & -0.16 & -3.61 & 2.67 & 6.47 & -0.59 & -6.06 \\
\hline Prec & -0.70 & 3.78 & 0.09 & -0.27 & -0.27 & 0.21 & -3.83 & -12.40 & -0.59 & 5.42 & 2.29 \\
\hline UVB & 3.97 & -1.95 & -3.36 & -2.90 & 0.73 & -0.53 & 15.80 & 9.99 & -6.06 & 2.29 & 75.90 \\
\hline Partial C.C. & dS & $d N$ & Diversif & Body Size & Elev & Lat & Temp & HR & NPP & Prec & UVB \\
\hline dS & -1.00 & 0.20 & -0.05 & -0.08 & -0.30 & -0.09 & 0.43 & 0.17 & 0.22 & 0.03 & -0.05 \\
\hline $\mathrm{dN}$ & 0.20 & -1.00 & 0.03 & -0.10 & 0.09 & -0.09 & -0.10 & 0.00 & 0.01 & -0.21 & 0.02 \\
\hline Diversif & -0.05 & 0.03 & -1.00 & -0.05 & 0.01 & -0.07 & 0.12 & 0.02 & -0.04 & -0.01 & 0.11 \\
\hline Body Size & -0.08 & -0.10 & -0.05 & -1.00 & 0.07 & 0.03 & 0.00 & 0.15 & -0.02 & 0.03 & 0.07 \\
\hline Elev & -0.30 & 0.09 & 0.01 & 0.07 & -1.00 & 0.06 & -0.21 & -0.16 & 0.09 & 0.10 & -0.07 \\
\hline Lat & -0.09 & -0.09 & -0.07 & 0.03 & 0.06 & -1.00 & 0.04 & 0.14 & 0.11 & -0.16 & 0.11 \\
\hline Temp & 0.43 & -0.10 & 0.12 & 0.00 & -0.21 & 0.04 & -1.00 & -0.13 & 0.17 & 0.18 & -0.20 \\
\hline$H R$ & 0.17 & 0.00 & 0.02 & 0.15 & -0.16 & 0.14 & -0.13 & -1.00 & -0.07 & 0.41 & -0.09 \\
\hline NPP & 0.22 & 0.01 & -0.04 & -0.02 & 0.09 & 0.11 & 0.17 & -0.07 & -1.00 & 0.10 & 0.28 \\
\hline Prec & 0.03 & -0.21 & -0.01 & 0.03 & 0.10 & -0.16 & 0.18 & 0.41 & 0.10 & -1.00 & -0.12 \\
\hline UVB & -0.05 & 0.02 & 0.11 & 0.07 & -0.07 & 0.11 & -0.20 & -0.09 & 0.28 & -0.12 & -1.00 \\
\hline Post. Prob. & dS & $d N$ & Diversif & Body Size & Elev & Lat & Temp & HR & NPP & Prec & UVB \\
\hline dS & - & 0.74 & 0.41 & 0.39 & 0.12 & 0.37 & 0.95 & 0.73 & 0.77 & 0.53 & 0.42 \\
\hline $\mathrm{dN}$ & 0.74 & - & 0.56 & 0.36 & 0.64 & 0.38 & 0.35 & 0.50 & 0.51 & 0.24 & 0.53 \\
\hline Diversif & 0.41 & 0.56 & - & 0.33 & 0.54 & 0.29 & 0.80 & 0.57 & 0.38 & 0.46 & 0.80 \\
\hline Body Size & 0.39 & 0.36 & 0.33 & - & 0.68 & 0.59 & 0.48 & 0.85 & 0.43 & 0.59 & 0.69 \\
\hline Elev & 0.12 & 0.64 & 0.54 & 0.68 & - & 0.66 & 0.15 & 0.16 & 0.70 & 0.74 & 0.32 \\
\hline Lat & 0.37 & 0.38 & 0.29 & 0.59 & 0.66 & - & 0.58 & 0.83 & 0.77 & 0.16 & 0.78 \\
\hline Temp & 0.95 & 0.35 & 0.80 & 0.48 & 0.15 & 0.58 & - & 0.22 & 0.82 & 0.84 & 0.13 \\
\hline $\mathrm{HR}$ & 0.73 & 0.50 & 0.57 & 0.85 & 0.16 & 0.83 & 0.22 & - & 0.34 & 0.98 & 0.27 \\
\hline NPP & 0.77 & 0.51 & 0.38 & 0.43 & 0.70 & 0.77 & 0.82 & 0.34 & - & 0.74 & 0.95 \\
\hline Prec & 0.53 & 0.24 & 0.46 & 0.59 & 0.74 & 0.16 & 0.84 & 0.98 & 0.74 & - & 0.22 \\
\hline UVB & 0.42 & 0.53 & 0.80 & 0.69 & 0.32 & 0.78 & 0.13 & 0.27 & 0.95 & 0.22 & - \\
\hline
\end{tabular}




\begin{tabular}{|c|c|c|c|c|c|c|c|c|c|c|}
\hline \multicolumn{11}{|c|}{ NUCLEAR CODING-GENES: dN ANALYSIS 2 (Controlling for Temperature) } \\
\hline Covariances & dS & $\mathrm{dN}$ & Diversif & Body Size & Elev & Lat & HR & NPP & Prec & UVB \\
\hline dS & 0.08 & 0.05 & 0.00 & -0.01 & -0.08 & -0.06 & 0.01 & 0.02 & 0.01 & 0.00 \\
\hline $\mathrm{dN}$ & 0.05 & 0.21 & 0.01 & -0.02 & 0.00 & -0.10 & 0.00 & 0.00 & -0.06 & 0.00 \\
\hline Diversif & 0.00 & 0.01 & 0.10 & 0.00 & 0.00 & -0.04 & 0.00 & 0.00 & -0.01 & 0.00 \\
\hline Body Size & -0.01 & -0.02 & 0.00 & 0.06 & 0.02 & 0.05 & 0.01 & 0.00 & 0.02 & 0.00 \\
\hline Elev & -0.08 & 0.00 & 0.00 & 0.02 & 1.21 & 0.19 & -0.02 & 0.02 & 0.00 & 0.00 \\
\hline Lat & -0.06 & -0.10 & -0.04 & 0.05 & 0.19 & 4.87 & 0.01 & 0.18 & -0.13 & 0.06 \\
\hline$H R$ & 0.01 & 0.00 & 0.00 & 0.01 & -0.02 & 0.01 & 0.01 & 0.00 & 0.03 & 0.00 \\
\hline NPP & 0.02 & 0.00 & 0.00 & 0.00 & 0.02 & 0.18 & 0.00 & 0.27 & 0.01 & 0.03 \\
\hline Prec & 0.01 & -0.06 & -0.01 & 0.02 & 0.00 & -0.13 & 0.03 & 0.01 & 0.40 & -0.02 \\
\hline UVB & 0.00 & 0.00 & 0.00 & 0.00 & 0.00 & 0.06 & 0.00 & 0.03 & -0.02 & 0.02 \\
\hline Correl. Coeff. & dS & $\mathbf{d N}$ & Diversif & Body Size & Elev & Lat & HR & NPP & Prec & UVB \\
\hline dS & 1.00 & 0.20 & -0.03 & -0.10 & -0.28 & -0.10 & 0.17 & 0.15 & 0.05 & -0.02 \\
\hline $\mathrm{dN}$ & 0.20 & 1.00 & 0.03 & -0.13 & 0.01 & -0.07 & -0.09 & 0.04 & -0.20 & 0.04 \\
\hline Diversif & -0.03 & 0.03 & 1.00 & -0.05 & 0.01 & -0.06 & -0.02 & -0.02 & -0.04 & 0.10 \\
\hline Body Size & -0.10 & -0.13 & -0.05 & 1.00 & 0.07 & 0.09 & 0.20 & -0.02 & 0.16 & 0.04 \\
\hline Elev & -0.28 & 0.01 & 0.01 & 0.07 & 1.00 & 0.08 & -0.21 & 0.04 & 0.00 & -0.01 \\
\hline Lat & -0.10 & -0.07 & -0.06 & 0.09 & 0.08 & 1.00 & 0.05 & 0.15 & -0.10 & 0.19 \\
\hline $\mathrm{HR}$ & 0.17 & -0.09 & -0.02 & 0.20 & -0.21 & 0.05 & 1.00 & -0.04 & 0.50 & -0.17 \\
\hline NPP & 0.15 & 0.04 & -0.02 & -0.02 & 0.04 & 0.15 & -0.04 & 1.00 & 0.03 & 0.32 \\
\hline Prec & 0.05 & -0.20 & -0.04 & 0.16 & 0.00 & -0.10 & 0.50 & 0.03 & 1.00 & -0.20 \\
\hline UVB & -0.02 & 0.04 & 0.10 & 0.04 & -0.01 & 0.19 & -0.17 & 0.32 & -0.20 & 1.00 \\
\hline Post. Prob. & dS & $\mathrm{dN}$ & Diversif & Body Size & Elev & Lat & HR & NPP & Prec & UVB \\
\hline $\mathrm{dS}$ & - & 0.72 & 0.43 & 0.35 & 0.11 & 0.35 & 0.73 & 0.70 & 0.57 & 0.46 \\
\hline $\mathrm{dN}$ & 0.72 & - & 0.58 & 0.29 & 0.51 & 0.39 & 0.36 & 0.54 & 0.25 & 0.55 \\
\hline Diversif & 0.43 & 0.58 & - & 0.33 & 0.55 & 0.30 & 0.42 & 0.41 & 0.35 & 0.83 \\
\hline Body Size & 0.35 & 0.29 & 0.33 & - & 0.73 & 0.79 & 0.96 & 0.45 & 0.91 & 0.63 \\
\hline Elev & 0.11 & 0.51 & 0.55 & 0.73 & - & 0.76 & 0.03 & 0.65 & 0.48 & 0.48 \\
\hline Lat & 0.35 & 0.39 & 0.30 & 0.79 & 0.76 & - & 0.67 & 0.92 & 0.19 & 0.96 \\
\hline $\mathrm{HR}$ & 0.73 & 0.36 & 0.42 & 0.96 & 0.03 & 0.67 & - & 0.37 & 1.00 & 0.06 \\
\hline NPP & 0.70 & 0.54 & 0.41 & 0.45 & 0.65 & 0.92 & 0.37 & - & 0.60 & 1.00 \\
\hline Prec & 0.57 & 0.25 & 0.35 & 0.91 & 0.48 & 0.19 & 1.00 & 0.60 & - & 0.03 \\
\hline UVB & 0.46 & 0.55 & 0.83 & 0.63 & 0.48 & 0.96 & 0.06 & 1.00 & 0.03 & - \\
\hline
\end{tabular}




\begin{tabular}{|c|c|c|c|c|c|c|c|c|c|c|c|}
\hline \multicolumn{12}{|c|}{ NUCLEAR CODING-GENES: $\omega$ ANALYSIS 1} \\
\hline Covariances & dS & $\omega$ & Diversif & Body Size & Elev & Lat & Temp & HR & NPP & Prec & UVB \\
\hline $\mathrm{dS}$ & 0.43 & 0.04 & 0.02 & -0.01 & -0.24 & -0.18 & 0.05 & 0.01 & 0.04 & 0.02 & -0.01 \\
\hline$\omega$ & 0.04 & 0.28 & 0.01 & -0.01 & 0.20 & -0.01 & -0.04 & -0.01 & -0.05 & -0.13 & 0.01 \\
\hline Diversif & 0.02 & 0.01 & 0.11 & 0.00 & -0.02 & -0.05 & 0.01 & 0.00 & 0.00 & 0.00 & 0.00 \\
\hline Body Size & -0.01 & -0.01 & 0.00 & 0.07 & 0.04 & 0.06 & 0.00 & 0.01 & -0.01 & 0.02 & 0.01 \\
\hline Elev & -0.24 & 0.20 & -0.02 & 0.04 & 1.62 & 0.37 & -0.13 & -0.04 & -0.10 & -0.17 & 0.03 \\
\hline Lat & -0.18 & -0.01 & -0.05 & 0.06 & 0.37 & 4.81 & -0.05 & 0.00 & 0.12 & -0.19 & 0.07 \\
\hline Temp & 0.05 & -0.04 & 0.01 & 0.00 & -0.13 & -0.05 & 0.04 & 0.00 & 0.04 & 0.05 & -0.01 \\
\hline $\mathrm{HR}$ & 0.01 & -0.01 & 0.00 & 0.01 & -0.04 & 0.00 & 0.00 & 0.01 & 0.00 & 0.04 & 0.00 \\
\hline NPP & 0.04 & -0.05 & 0.00 & -0.01 & -0.10 & 0.12 & 0.04 & 0.00 & 0.29 & 0.05 & 0.02 \\
\hline Prec & 0.02 & -0.13 & 0.00 & 0.02 & -0.17 & -0.19 & 0.05 & 0.04 & 0.05 & 0.44 & -0.03 \\
\hline UVB & -0.01 & 0.01 & 0.00 & 0.01 & 0.03 & 0.07 & -0.01 & 0.00 & 0.02 & -0.03 & 0.03 \\
\hline Correl. Coeff. & dS & $\omega$ & Diversif & Body Size & Elev & Lat & Temp & HR & NPP & Prec & UVB \\
\hline dS & 1.00 & -0.10 & 0.06 & -0.15 & -0.38 & -0.19 & 0.46 & 0.19 & 0.19 & 0.10 & -0.17 \\
\hline$\omega$ & -0.10 & 1.00 & 0.03 & -0.05 & 0.34 & -0.01 & -0.42 & -0.28 & -0.20 & -0.41 & 0.16 \\
\hline Diversif & 0.06 & 0.03 & 1.00 & -0.06 & -0.04 & -0.07 & 0.11 & 0.00 & 0.01 & 0.00 & 0.06 \\
\hline Body Size & -0.15 & -0.05 & -0.06 & 1.00 & 0.11 & 0.10 & -0.11 & 0.17 & -0.06 & 0.10 & 0.06 \\
\hline Elev & -0.38 & 0.34 & -0.04 & 0.11 & 1.00 & 0.13 & -0.52 & -0.29 & -0.14 & -0.20 & 0.15 \\
\hline Lat & -0.19 & -0.01 & -0.07 & 0.10 & 0.13 & 1.00 & -0.13 & 0.02 & 0.10 & -0.13 & 0.21 \\
\hline Temp & 0.46 & -0.42 & 0.11 & -0.11 & -0.52 & -0.13 & 1.00 & 0.22 & 0.34 & 0.37 & -0.29 \\
\hline $\mathrm{HR}$ & 0.19 & -0.28 & 0.00 & 0.17 & -0.29 & 0.02 & 0.22 & 1.00 & 0.03 & 0.54 & -0.22 \\
\hline NPP & 0.19 & -0.20 & 0.01 & -0.06 & -0.14 & 0.10 & 0.34 & 0.03 & 1.00 & 0.14 & 0.19 \\
\hline Prec & 0.10 & -0.41 & 0.00 & 0.10 & -0.20 & -0.13 & 0.37 & 0.54 & 0.14 & 1.00 & -0.29 \\
\hline UVB & -0.17 & 0.16 & 0.06 & 0.06 & 0.15 & 0.21 & -0.29 & -0.22 & 0.19 & -0.29 & 1.00 \\
\hline Post. Prob. & dS & $\omega$ & Diversif & Body Size & Elev & Lat & Temp & HR & NPP & Prec & UVB \\
\hline dS & - & 0.37 & 0.67 & 0.23 & 0.03 & 0.20 & 0.98 & 0.79 & 0.79 & 0.67 & 0.21 \\
\hline$\omega$ & 0.37 & - & 0.56 & 0.40 & 0.92 & 0.48 & 0.04 & 0.14 & 0.18 & 0.06 & 0.76 \\
\hline Diversif & 0.67 & 0.56 & - & 0.28 & 0.35 & 0.25 & 0.83 & 0.51 & 0.55 & 0.50 & 0.72 \\
\hline Body Size & 0.23 & 0.40 & 0.28 & - & 0.85 & 0.81 & 0.16 & 0.93 & 0.29 & 0.81 & 0.70 \\
\hline Elev & 0.03 & 0.92 & 0.35 & 0.85 & - & 0.88 & 0.00 & 0.01 & 0.10 & 0.04 & 0.90 \\
\hline Lat & 0.20 & 0.48 & 0.25 & 0.81 & 0.88 & - & 0.13 & 0.56 & 0.81 & 0.12 & 0.97 \\
\hline Temp & 0.98 & 0.04 & 0.83 & 0.16 & 0.00 & 0.13 & - & 0.98 & 1.00 & 1.00 & 0.01 \\
\hline $\mathrm{HR}$ & 0.79 & 0.14 & 0.51 & 0.93 & 0.01 & 0.56 & 0.98 & - & 0.63 & 1.00 & 0.03 \\
\hline NPP & 0.79 & 0.18 & 0.55 & 0.29 & 0.10 & 0.81 & 1.00 & 0.63 & - & 0.90 & 0.96 \\
\hline Prec & 0.67 & 0.06 & 0.50 & 0.81 & 0.04 & 0.12 & 1.00 & 1.00 & 0.90 & - & 0.01 \\
\hline UVB & 0.21 & 0.76 & 0.72 & 0.70 & 0.90 & 0.97 & 0.01 & 0.03 & 0.96 & 0.01 & - \\
\hline
\end{tabular}




\begin{tabular}{|c|c|c|c|c|c|c|c|c|c|c|c|}
\hline Precisions & dS & $\omega$ & Diversif & Body Size & Elev & Lat & Temp & HR & NPP & Prec & UVB \\
\hline dS & - & 0.37 & 0.67 & 0.23 & 0.03 & 0.20 & 0.98 & 0.79 & 0.79 & 0.67 & 0.21 \\
\hline$\omega$ & 0.37 & - & 0.56 & 0.40 & 0.92 & 0.48 & 0.04 & 0.14 & 0.18 & 0.06 & 0.76 \\
\hline Diversif & 0.67 & 0.56 & - & 0.28 & 0.35 & 0.25 & 0.83 & 0.51 & 0.55 & 0.50 & 0.72 \\
\hline Body Size & 0.23 & 0.40 & 0.28 & - & 0.85 & 0.81 & 0.16 & 0.93 & 0.29 & 0.81 & 0.70 \\
\hline Elev & 0.03 & 0.92 & 0.35 & 0.85 & - & 0.88 & 0.00 & 0.01 & 0.10 & 0.04 & 0.90 \\
\hline Lat & 0.20 & 0.48 & 0.25 & 0.81 & 0.88 & - & 0.13 & 0.56 & 0.81 & 0.12 & 0.97 \\
\hline Temp & 0.98 & 0.04 & 0.83 & 0.16 & 0.00 & 0.13 & - & 0.98 & 1.00 & 1.00 & 0.01 \\
\hline $\mathrm{HR}$ & 0.79 & 0.14 & 0.51 & 0.93 & 0.01 & 0.56 & 0.98 & - & 0.63 & 1.00 & 0.03 \\
\hline NPP & 0.79 & 0.18 & 0.55 & 0.29 & 0.10 & 0.81 & 1.00 & 0.63 & - & 0.90 & 0.96 \\
\hline Prec & 0.67 & 0.06 & 0.50 & 0.81 & 0.04 & 0.12 & 1.00 & 1.00 & 0.90 & - & 0.01 \\
\hline UVB & 0.21 & 0.76 & 0.72 & 0.70 & 0.90 & 0.97 & 0.01 & 0.03 & 0.96 & 0.01 & - \\
\hline Partial C.C. & dS & $\omega$ & Diversif & Body Size & Elev & Lat & Temp & HR & NPP & Prec & UVB \\
\hline dS & -1.00 & 0.17 & -0.01 & -0.10 & -0.19 & -0.16 & 0.36 & 0.20 & 0.13 & -0.12 & -0.05 \\
\hline$\omega$ & 0.17 & -1.00 & 0.06 & -0.07 & 0.23 & -0.09 & -0.30 & -0.05 & -0.09 & -0.28 & 0.05 \\
\hline Diversif & -0.01 & 0.06 & -1.00 & -0.05 & 0.01 & -0.07 & 0.12 & 0.02 & -0.04 & 0.00 & 0.11 \\
\hline Body Size & -0.10 & -0.07 & -0.05 & -1.00 & 0.09 & 0.03 & -0.02 & 0.15 & -0.05 & 0.02 & 0.07 \\
\hline Elev & -0.19 & 0.23 & 0.01 & 0.09 & -1.00 & 0.08 & -0.25 & -0.18 & 0.06 & 0.12 & -0.07 \\
\hline Lat & -0.16 & -0.09 & -0.07 & 0.03 & 0.08 & -1.00 & 0.02 & 0.15 & 0.10 & -0.18 & 0.11 \\
\hline Temp & 0.36 & -0.30 & 0.12 & -0.02 & -0.25 & 0.02 & -1.00 & -0.13 & 0.23 & 0.16 & -0.20 \\
\hline $\mathrm{HR}$ & 0.20 & -0.05 & 0.02 & 0.15 & -0.18 & 0.15 & -0.13 & -1.00 & -0.07 & 0.42 & -0.08 \\
\hline NPP & 0.13 & -0.09 & -0.04 & -0.05 & 0.06 & 0.10 & 0.23 & -0.07 & -1.00 & 0.08 & 0.28 \\
\hline Prec & -0.12 & -0.28 & 0.00 & 0.02 & 0.12 & -0.18 & 0.16 & 0.42 & 0.08 & -1.00 & -0.12 \\
\hline UVB & -0.05 & 0.05 & 0.11 & 0.07 & -0.07 & 0.11 & -0.20 & -0.08 & 0.28 & -0.12 & -1.00 \\
\hline Post. Prob. & dS & $\omega$ & Diversif & Body Size & Elev & Lat & Temp & HR & NPP & Prec & UVB \\
\hline dS & - & 0.73 & 0.49 & 0.34 & 0.22 & 0.26 & 0.93 & 0.78 & 0.68 & 0.31 & 0.41 \\
\hline$\omega$ & 0.73 & - & 0.62 & 0.40 & 0.79 & 0.37 & 0.13 & 0.43 & 0.38 & 0.16 & 0.56 \\
\hline Diversif & 0.49 & 0.62 & - & 0.33 & 0.54 & 0.29 & 0.81 & 0.57 & 0.39 & 0.50 & 0.82 \\
\hline Body Size & 0.34 & 0.40 & 0.33 & - & 0.75 & 0.59 & 0.44 & 0.87 & 0.36 & 0.57 & 0.71 \\
\hline Elev & 0.22 & 0.79 & 0.54 & 0.75 & - & 0.70 & 0.11 & 0.13 & 0.66 & 0.78 & 0.31 \\
\hline Lat & 0.26 & 0.37 & 0.29 & 0.59 & 0.70 & - & 0.55 & 0.84 & 0.77 & 0.12 & 0.80 \\
\hline Temp & 0.93 & 0.13 & 0.81 & 0.44 & 0.11 & 0.55 & - & 0.21 & 0.89 & 0.82 & 0.12 \\
\hline$H R$ & 0.78 & 0.43 & 0.57 & 0.87 & 0.13 & 0.84 & 0.21 & - & 0.33 & 0.99 & 0.28 \\
\hline NPP & 0.68 & 0.38 & 0.39 & 0.36 & 0.66 & 0.77 & 0.89 & 0.33 & - & 0.70 & 0.97 \\
\hline Prec & 0.31 & 0.16 & 0.50 & 0.57 & 0.78 & 0.12 & 0.82 & 0.99 & 0.70 & - & 0.21 \\
\hline UVB & 0.41 & 0.56 & 0.82 & 0.71 & 0.31 & 0.80 & 0.12 & 0.28 & 0.97 & 0.21 & - \\
\hline
\end{tabular}




\begin{tabular}{|c|c|c|c|c|c|c|c|c|c|c|}
\hline \multicolumn{11}{|c|}{ NUCLEAR CODING-GENES: $\omega$ ANALYSIS 1 (Controlling for Temperature) } \\
\hline Covariances & dS & $\omega$ & Diversif & Body Size & Elev & Lat & HR & NPP & Prec & UVB \\
\hline dS & 0.33 & 0.07 & 0.01 & -0.01 & -0.09 & -0.13 & 0.00 & 0.00 & -0.03 & 0.00 \\
\hline$\omega$ & 0.07 & 0.22 & 0.01 & -0.01 & 0.07 & -0.07 & -0.01 & -0.01 & -0.08 & 0.00 \\
\hline Diversif & 0.01 & 0.01 & 0.10 & 0.00 & 0.00 & -0.04 & 0.00 & -0.01 & -0.01 & 0.00 \\
\hline Body Size & -0.01 & -0.01 & 0.00 & 0.06 & 0.02 & 0.05 & 0.01 & 0.00 & 0.02 & 0.00 \\
\hline Elev & -0.09 & 0.07 & 0.00 & 0.02 & 1.16 & 0.18 & -0.02 & 0.02 & 0.00 & 0.00 \\
\hline Lat & -0.13 & -0.07 & -0.04 & 0.05 & 0.18 & 4.66 & 0.01 & 0.17 & -0.12 & 0.06 \\
\hline$H R$ & 0.00 & -0.01 & 0.00 & 0.01 & -0.02 & 0.01 & 0.01 & 0.00 & 0.03 & 0.00 \\
\hline NPP & 0.00 & -0.01 & -0.01 & 0.00 & 0.02 & 0.17 & 0.00 & 0.26 & 0.01 & 0.02 \\
\hline Prec & -0.03 & -0.08 & -0.01 & 0.02 & 0.00 & -0.12 & 0.03 & 0.01 & 0.38 & -0.02 \\
\hline UVB & 0.00 & 0.00 & 0.00 & 0.00 & 0.00 & 0.06 & 0.00 & 0.02 & -0.02 & 0.02 \\
\hline Correl. Coeff. & dS & $\omega$ & Diversif & Body Size & Elev & Lat & HR & NPP & Prec & UVB \\
\hline dS & 1.00 & 0.13 & 0.02 & -0.11 & -0.19 & -0.15 & 0.11 & 0.04 & -0.08 & -0.04 \\
\hline$\omega$ & 0.13 & 1.00 & 0.08 & -0.12 & 0.16 & -0.07 & -0.21 & -0.07 & -0.31 & 0.05 \\
\hline Diversif & 0.02 & 0.08 & 1.00 & -0.05 & 0.01 & -0.06 & -0.02 & -0.02 & -0.04 & 0.10 \\
\hline Body Size & -0.11 & -0.12 & -0.05 & 1.00 & 0.06 & 0.09 & 0.20 & -0.02 & 0.16 & 0.03 \\
\hline Elev & -0.19 & 0.16 & 0.01 & 0.06 & 1.00 & 0.08 & -0.20 & 0.04 & 0.00 & -0.01 \\
\hline Lat & -0.15 & -0.07 & -0.06 & 0.09 & 0.08 & 1.00 & 0.05 & 0.15 & -0.09 & 0.19 \\
\hline $\mathrm{HR}$ & 0.11 & -0.21 & -0.02 & 0.20 & -0.20 & 0.05 & 1.00 & -0.04 & 0.50 & -0.17 \\
\hline NPP & 0.04 & -0.07 & -0.02 & -0.02 & 0.04 & 0.15 & -0.04 & 1.00 & 0.02 & 0.32 \\
\hline Prec & -0.08 & -0.31 & -0.04 & 0.16 & 0.00 & -0.09 & 0.50 & 0.02 & 1.00 & -0.20 \\
\hline UVB & -0.04 & 0.05 & 0.10 & 0.03 & -0.01 & 0.19 & -0.17 & 0.32 & -0.20 & 1.00 \\
\hline Post. Prob. & dS & $\omega$ & Diversif & Body Size & Elev & Lat & HR & NPP & Prec & UVB \\
\hline dS & - & 0.66 & 0.55 & 0.31 & 0.18 & 0.26 & 0.66 & 0.55 & 0.37 & 0.43 \\
\hline$\omega$ & 0.66 & - & 0.67 & 0.30 & 0.73 & 0.38 & 0.22 & 0.40 & 0.13 & 0.57 \\
\hline Diversif & 0.55 & 0.67 & - & 0.31 & 0.55 & 0.29 & 0.42 & 0.42 & 0.35 & 0.82 \\
\hline Body Size & 0.31 & 0.30 & 0.31 & - & 0.72 & 0.78 & 0.96 & 0.41 & 0.91 & 0.60 \\
\hline Elev & 0.18 & 0.73 & 0.55 & 0.72 & - & 0.75 & 0.03 & 0.66 & 0.49 & 0.49 \\
\hline Lat & 0.26 & 0.38 & 0.29 & 0.78 & 0.75 & - & 0.67 & 0.92 & 0.21 & 0.95 \\
\hline$H R$ & 0.66 & 0.22 & 0.42 & 0.96 & 0.03 & 0.67 & - & 0.34 & 1.00 & 0.07 \\
\hline NPP & 0.55 & 0.40 & 0.42 & 0.41 & 0.66 & 0.92 & 0.34 & - & 0.56 & 1.00 \\
\hline Prec & 0.37 & 0.13 & 0.35 & 0.91 & 0.49 & 0.21 & 1.00 & 0.56 & - & 0.04 \\
\hline UVB & 0.43 & 0.57 & 0.82 & 0.60 & 0.49 & 0.95 & 0.07 & 1.00 & 0.04 & - \\
\hline
\end{tabular}




\begin{tabular}{|c|c|c|c|c|c|c|c|c|c|c|c|}
\hline \multicolumn{12}{|c|}{ NUCLEAR CODING-GENES: $\omega$ ANALYSIS 2} \\
\hline Covariances & dS & $\omega$ & Diversif & Body Size & Elev & Lat & Temp & HR & NPP & Prec & UVB \\
\hline dS & 0.35 & 0.03 & 0.01 & -0.02 & -0.24 & -0.22 & 0.05 & 0.01 & 0.05 & 0.04 & -0.01 \\
\hline$\omega$ & 0.03 & 0.30 & 0.01 & -0.01 & 0.21 & -0.03 & -0.04 & -0.02 & -0.05 & -0.13 & 0.01 \\
\hline Diversif & 0.01 & 0.01 & 0.11 & 0.00 & -0.02 & -0.05 & 0.01 & 0.00 & 0.01 & 0.00 & 0.01 \\
\hline Body Size & -0.02 & -0.01 & 0.00 & 0.06 & 0.04 & 0.06 & 0.00 & 0.00 & -0.01 & 0.02 & 0.00 \\
\hline Elev & -0.24 & 0.21 & -0.02 & 0.04 & 1.62 & 0.37 & -0.13 & -0.04 & -0.10 & -0.17 & 0.03 \\
\hline Lat & -0.22 & -0.03 & -0.05 & 0.06 & 0.37 & 4.83 & -0.05 & 0.00 & 0.13 & -0.19 & 0.07 \\
\hline Temp & 0.05 & -0.04 & 0.01 & 0.00 & -0.13 & -0.05 & 0.04 & 0.01 & 0.04 & 0.05 & -0.01 \\
\hline $\mathrm{HR}$ & 0.01 & -0.02 & 0.00 & 0.00 & -0.04 & 0.00 & 0.01 & 0.01 & 0.00 & 0.04 & 0.00 \\
\hline NPP & 0.05 & -0.05 & 0.01 & -0.01 & -0.10 & 0.13 & 0.04 & 0.00 & 0.30 & 0.05 & 0.02 \\
\hline Prec & 0.04 & -0.13 & 0.00 & 0.02 & -0.17 & -0.19 & 0.05 & 0.04 & 0.05 & 0.45 & -0.03 \\
\hline UVB & -0.01 & 0.01 & 0.01 & 0.00 & 0.03 & 0.07 & -0.01 & 0.00 & 0.02 & -0.03 & 0.03 \\
\hline Correl. Coeff. & dS & $\omega$ & Diversif & Body Size & Elev & Lat & Temp & HR & NPP & Prec & UVB \\
\hline dS & 1.00 & -0.08 & 0.07 & -0.16 & -0.38 & -0.20 & 0.46 & 0.20 & 0.19 & 0.11 & -0.17 \\
\hline$\omega$ & -0.08 & 1.00 & 0.02 & -0.07 & 0.34 & -0.02 & -0.42 & -0.28 & -0.20 & -0.41 & 0.16 \\
\hline Diversif & 0.07 & 0.02 & 1.00 & -0.06 & -0.04 & -0.07 & 0.10 & 0.00 & 0.01 & 0.00 & 0.07 \\
\hline Body Size & -0.16 & -0.07 & -0.06 & 1.00 & 0.12 & 0.10 & -0.11 & 0.17 & -0.06 & 0.10 & 0.06 \\
\hline Elev & -0.38 & 0.34 & -0.04 & 0.12 & 1.00 & 0.13 & -0.52 & -0.29 & -0.15 & -0.20 & 0.15 \\
\hline Lat & -0.20 & -0.02 & -0.07 & 0.10 & 0.13 & 1.00 & -0.13 & 0.02 & 0.10 & -0.13 & 0.21 \\
\hline Temp & 0.46 & -0.42 & 0.10 & -0.11 & -0.52 & -0.13 & 1.00 & 0.22 & 0.34 & 0.37 & -0.28 \\
\hline$H R$ & 0.20 & -0.28 & 0.00 & 0.17 & -0.29 & 0.02 & 0.22 & 1.00 & 0.03 & 0.54 & -0.23 \\
\hline NPP & 0.19 & -0.20 & 0.01 & -0.06 & -0.15 & 0.10 & 0.34 & 0.03 & 1.00 & 0.14 & 0.19 \\
\hline Prec & 0.11 & -0.41 & 0.00 & 0.10 & -0.20 & -0.13 & 0.37 & 0.54 & 0.14 & 1.00 & -0.29 \\
\hline UVB & -0.17 & 0.16 & 0.07 & 0.06 & 0.15 & 0.21 & -0.28 & -0.23 & 0.19 & -0.29 & 1.00 \\
\hline Post. Prob. & dS & $\omega$ & Diversif & Body Size & Elev & Lat & Temp & HR & NPP & Prec & UVB \\
\hline dS & - & 0.40 & 0.68 & 0.20 & 0.03 & 0.19 & 0.98 & 0.80 & 0.79 & 0.68 & 0.21 \\
\hline$\omega$ & 0.40 & - & 0.55 & 0.38 & 0.93 & 0.46 & 0.04 & 0.13 & 0.19 & 0.05 & 0.76 \\
\hline Diversif & 0.68 & 0.55 & - & 0.29 & 0.34 & 0.25 & 0.81 & 0.50 & 0.55 & 0.50 & 0.73 \\
\hline Body Size & 0.20 & 0.38 & 0.29 & - & 0.85 & 0.82 & 0.16 & 0.94 & 0.31 & 0.81 & 0.70 \\
\hline Elev & 0.03 & 0.93 & 0.34 & 0.85 & - & 0.88 & 0.00 & 0.00 & 0.10 & 0.04 & 0.90 \\
\hline Lat & 0.19 & 0.46 & 0.25 & 0.82 & 0.88 & - & 0.12 & 0.57 & 0.81 & 0.13 & 0.97 \\
\hline Temp & 0.98 & 0.04 & 0.81 & 0.16 & 0.00 & 0.12 & - & 0.98 & 1.00 & 1.00 & 0.01 \\
\hline$H R$ & 0.80 & 0.13 & 0.50 & 0.94 & 0.00 & 0.57 & 0.98 & - & 0.63 & 1.00 & 0.02 \\
\hline NPP & 0.79 & 0.19 & 0.55 & 0.31 & 0.10 & 0.81 & 1.00 & 0.63 & - & 0.90 & 0.96 \\
\hline Prec & 0.68 & 0.05 & 0.50 & 0.81 & 0.04 & 0.13 & 1.00 & 1.00 & 0.90 & - & 0.01 \\
\hline UVB & 0.21 & 0.76 & 0.73 & 0.70 & 0.90 & 0.97 & 0.01 & 0.02 & 0.96 & 0.01 & - \\
\hline
\end{tabular}




\begin{tabular}{|c|c|c|c|c|c|c|c|c|c|c|c|}
\hline Precisions & dS & $\omega$ & Diversif & Body Size & Elev & Lat & Temp & HR & NPP & Prec & UVB \\
\hline dS & 19.20 & -2.44 & 0.22 & 2.62 & 1.11 & 0.39 & -15.10 & -11.30 & -1.87 & 1.09 & 2.34 \\
\hline$\omega$ & -2.44 & 55.00 & -0.89 & 1.65 & -1.50 & 0.25 & 15.20 & 4.92 & 1.12 & 3.69 & -2.93 \\
\hline Diversif & 0.22 & -0.89 & 12.90 & 0.87 & -0.03 & 0.14 & -3.82 & -0.98 & 0.34 & -0.04 & -3.41 \\
\hline Body Size & 2.62 & 1.65 & 0.87 & 25.10 & -0.54 & -0.07 & 1.09 & -10.70 & 0.57 & -0.11 & -2.85 \\
\hline Elev & 1.11 & -1.50 & -0.03 & -0.54 & 1.40 & -0.05 & 2.52 & 2.70 & -0.20 & -0.38 & 0.77 \\
\hline Lat & 0.39 & 0.25 & 0.14 & -0.07 & -0.05 & 0.33 & -0.10 & -1.09 & -0.15 & 0.27 & -0.54 \\
\hline Temp & -15.10 & 15.20 & -3.82 & 1.09 & 2.52 & -0.10 & 88.80 & 17.50 & -4.98 & -3.44 & 15.70 \\
\hline $\mathrm{HR}$ & -11.30 & 4.92 & -0.98 & -10.70 & 2.70 & -1.09 & 17.50 & 185.00 & 2.44 & -13.80 & 9.12 \\
\hline NPP & -1.87 & 1.12 & 0.34 & 0.57 & -0.20 & -0.15 & -4.98 & 2.44 & 6.33 & -0.47 & -6.33 \\
\hline Prec & 1.09 & 3.69 & -0.04 & -0.11 & -0.38 & 0.27 & -3.44 & -13.80 & -0.47 & 5.93 & 2.42 \\
\hline UVB & 2.34 & -2.93 & -3.41 & -2.85 & 0.77 & -0.54 & 15.70 & 9.12 & -6.33 & 2.42 & 76.30 \\
\hline Partial C.C. & dS & $\omega$ & Diversif & Body Size & Elev & Lat & Temp & HR & NPP & Prec & UVB \\
\hline dS & -1.00 & 0.19 & 0.00 & -0.11 & -0.19 & -0.16 & 0.35 & 0.20 & 0.14 & -0.12 & -0.06 \\
\hline$\omega$ & 0.19 & -1.00 & 0.06 & -0.08 & 0.24 & -0.10 & -0.29 & -0.06 & -0.09 & -0.29 & 0.05 \\
\hline Diversif & 0.00 & 0.06 & -1.00 & -0.05 & 0.01 & -0.07 & 0.11 & 0.02 & -0.04 & 0.00 & 0.11 \\
\hline Body Size & -0.11 & -0.08 & -0.05 & -1.00 & 0.09 & 0.03 & -0.02 & 0.16 & -0.05 & 0.02 & 0.07 \\
\hline Elev & -0.19 & 0.24 & 0.01 & 0.09 & -1.00 & 0.08 & -0.25 & -0.18 & 0.06 & 0.13 & -0.07 \\
\hline Lat & -0.16 & -0.10 & -0.07 & 0.03 & 0.08 & -1.00 & 0.02 & 0.14 & 0.11 & -0.18 & 0.11 \\
\hline Temp & 0.35 & -0.29 & 0.11 & -0.02 & -0.25 & 0.02 & -1.00 & -0.13 & 0.23 & 0.16 & -0.20 \\
\hline HR & 0.20 & -0.06 & 0.02 & 0.16 & -0.18 & 0.14 & -0.13 & -1.00 & -0.07 & 0.42 & -0.08 \\
\hline NPP & 0.14 & -0.09 & -0.04 & -0.05 & 0.06 & 0.11 & 0.23 & -0.07 & -1.00 & 0.08 & 0.29 \\
\hline Prec & -0.12 & -0.29 & 0.00 & 0.02 & 0.13 & -0.18 & 0.16 & 0.42 & 0.08 & -1.00 & -0.12 \\
\hline UVB & -0.06 & 0.05 & 0.11 & 0.07 & -0.07 & 0.11 & -0.20 & -0.08 & 0.29 & -0.12 & -1.00 \\
\hline Post. Prob. & dS & $\omega$ & Diversif & Body Size & Elev & Lat & Temp & HR & NPP & Prec & UVB \\
\hline dS & - & 0.75 & 0.50 & 0.33 & 0.21 & 0.27 & 0.92 & 0.79 & 0.69 & 0.32 & 0.39 \\
\hline$\omega$ & 0.75 & - & 0.61 & 0.38 & 0.81 & 0.36 & 0.14 & 0.42 & 0.37 & 0.15 & 0.57 \\
\hline Diversif & 0.50 & 0.61 & - & 0.35 & 0.54 & 0.29 & 0.79 & 0.56 & 0.37 & 0.51 & 0.82 \\
\hline Body Size & 0.33 & 0.38 & 0.35 & - & 0.74 & 0.58 & 0.43 & 0.87 & 0.37 & 0.55 & 0.70 \\
\hline Elev & 0.21 & 0.81 & 0.54 & 0.74 & - & 0.71 & 0.11 & 0.13 & 0.65 & 0.79 & 0.30 \\
\hline Lat & 0.27 & 0.36 & 0.29 & 0.58 & 0.71 & - & 0.53 & 0.84 & 0.77 & 0.11 & 0.79 \\
\hline Temp & 0.92 & 0.14 & 0.79 & 0.43 & 0.11 & 0.53 & - & 0.21 & 0.89 & 0.82 & 0.12 \\
\hline $\mathrm{HR}$ & 0.79 & 0.42 & 0.56 & 0.87 & 0.13 & 0.84 & 0.21 & - & 0.33 & 0.99 & 0.28 \\
\hline NPP & 0.69 & 0.37 & 0.37 & 0.37 & 0.65 & 0.77 & 0.89 & 0.33 & - & 0.71 & 0.98 \\
\hline Prec & 0.32 & 0.15 & 0.51 & 0.55 & 0.79 & 0.11 & 0.82 & 0.99 & 0.71 & - & 0.21 \\
\hline UVB & 0.39 & 0.57 & 0.82 & 0.70 & 0.30 & 0.79 & 0.12 & 0.28 & 0.98 & 0.21 & - \\
\hline
\end{tabular}




\begin{tabular}{|c|c|c|c|c|c|c|c|c|c|c|}
\hline \multicolumn{11}{|c|}{ NUCLEAR CODING-GENES: $\omega$ ANALYSIS 2 (Controlling for Temperature) } \\
\hline Covariances & dS & $\omega$ & Diversif & Body Size & Elev & Lat & HR & NPP & Prec & UVB \\
\hline dS & 0.28 & 0.07 & 0.00 & -0.01 & -0.09 & -0.15 & 0.00 & 0.01 & -0.02 & 0.00 \\
\hline$\omega$ & 0.07 & 0.24 & 0.01 & -0.02 & 0.07 & -0.09 & -0.01 & -0.01 & -0.08 & 0.00 \\
\hline Diversif & 0.00 & 0.01 & 0.10 & 0.00 & 0.00 & -0.04 & 0.00 & 0.00 & -0.01 & 0.00 \\
\hline Body Size & -0.01 & -0.02 & 0.00 & 0.06 & 0.02 & 0.05 & 0.01 & 0.00 & 0.02 & 0.00 \\
\hline Elev & -0.09 & 0.07 & 0.00 & 0.02 & 1.16 & 0.18 & -0.02 & 0.02 & 0.00 & 0.00 \\
\hline Lat & -0.15 & -0.09 & -0.04 & 0.05 & 0.18 & 4.68 & 0.01 & 0.17 & -0.12 & 0.06 \\
\hline $\mathrm{HR}$ & 0.00 & -0.01 & 0.00 & 0.01 & -0.02 & 0.01 & 0.01 & 0.00 & 0.03 & 0.00 \\
\hline NPP & 0.01 & -0.01 & 0.00 & 0.00 & 0.02 & 0.17 & 0.00 & 0.26 & 0.01 & 0.02 \\
\hline Prec & -0.02 & -0.08 & -0.01 & 0.02 & 0.00 & -0.12 & 0.03 & 0.01 & 0.38 & -0.02 \\
\hline UVB & 0.00 & 0.00 & 0.00 & 0.00 & 0.00 & 0.06 & 0.00 & 0.02 & -0.02 & 0.02 \\
\hline Correl. Coeff. & dS & $\omega$ & Diversif & Body Size & Elev & Lat & HR & NPP & Prec & UVB \\
\hline $\mathrm{dS}$ & 1.00 & 0.14 & 0.02 & -0.13 & -0.19 & -0.16 & 0.11 & 0.05 & -0.08 & -0.05 \\
\hline$\omega$ & 0.14 & 1.00 & 0.07 & -0.13 & 0.16 & -0.09 & -0.22 & -0.06 & -0.31 & 0.05 \\
\hline Diversif & 0.02 & 0.07 & 1.00 & -0.05 & 0.01 & -0.06 & -0.03 & -0.02 & -0.04 & 0.10 \\
\hline Body Size & -0.13 & -0.13 & -0.05 & 1.00 & 0.07 & 0.09 & 0.20 & -0.02 & 0.16 & 0.03 \\
\hline Elev & -0.19 & 0.16 & 0.01 & 0.07 & 1.00 & 0.07 & -0.20 & 0.04 & 0.00 & -0.01 \\
\hline Lat & -0.16 & -0.09 & -0.06 & 0.09 & 0.07 & 1.00 & 0.05 & 0.16 & -0.09 & 0.19 \\
\hline$H R$ & 0.11 & -0.22 & -0.03 & 0.20 & -0.20 & 0.05 & 1.00 & -0.05 & 0.50 & -0.17 \\
\hline NPP & 0.05 & -0.06 & -0.02 & -0.02 & 0.04 & 0.16 & -0.05 & 1.00 & 0.02 & 0.32 \\
\hline Prec & -0.08 & -0.31 & -0.04 & 0.16 & 0.00 & -0.09 & 0.50 & 0.02 & 1.00 & -0.20 \\
\hline UVB & -0.05 & 0.05 & 0.10 & 0.03 & -0.01 & 0.19 & -0.17 & 0.32 & -0.20 & 1.00 \\
\hline Post. Prob. & dS & $\omega$ & Diversif & Body Size & Elev & Lat & HR & NPP & Prec & UVB \\
\hline $\mathrm{dS}$ & - & 0.69 & 0.56 & 0.28 & 0.19 & 0.26 & 0.66 & 0.56 & 0.39 & 0.41 \\
\hline$\omega$ & 0.69 & - & 0.67 & 0.29 & 0.74 & 0.36 & 0.21 & 0.40 & 0.12 & 0.58 \\
\hline Diversif & 0.56 & 0.67 & - & 0.32 & 0.55 & 0.29 & 0.41 & 0.42 & 0.35 & 0.83 \\
\hline Body Size & 0.28 & 0.29 & 0.32 & - & 0.73 & 0.79 & 0.97 & 0.43 & 0.91 & 0.60 \\
\hline Elev & 0.19 & 0.74 & 0.55 & 0.73 & - & 0.75 & 0.03 & 0.65 & 0.49 & 0.49 \\
\hline Lat & 0.26 & 0.36 & 0.29 & 0.79 & 0.75 & - & 0.68 & 0.92 & 0.22 & 0.95 \\
\hline$H R$ & 0.66 & 0.21 & 0.41 & 0.97 & 0.03 & 0.68 & - & 0.34 & 1.00 & 0.06 \\
\hline NPP & 0.56 & 0.40 & 0.42 & 0.43 & 0.65 & 0.92 & 0.34 & - & 0.57 & 1.00 \\
\hline Prec & 0.39 & 0.12 & 0.35 & 0.91 & 0.49 & 0.22 & 1.00 & 0.57 & - & 0.04 \\
\hline UVB & 0.41 & 0.58 & 0.83 & 0.60 & 0.49 & 0.95 & 0.06 & 1.00 & 0.04 & - \\
\hline
\end{tabular}




\section{REFERENCES}

1 Rojas-Runjaic, F. J. M., Infante-Rivero, E. E. \& Cabello, P. 2012 New records and distribution extensions of centrolenid frogs for Venezuela. Check List 8, 819-825.

2 Rivera-Correa, M. 2010 Amphibia, Centrolenidae, Centrolene antioquiense (Noble, 1920): New records and geographical distribution in Colombia. Check List 6, 220-221.

3 Almendáriz, A. \& Batallas, D. 2012 Nuevos datos sobre la Distribución, Historia Natural y el Canto de Centrolene condor Cisneros-Heredia y Morales-Mite 2008 (Amphibia: Anura: Centrolenidae). Politécnica 30, 42-53.

4 Rojas-Runjaic, F. J. M., Infante-Rivero, E. E., Señaris, J. C. \& Cabello, P. 2010 Amphibia, Anura, Centrolenidae, Centrolene daidaleum (RuizCarranza and Lynch, 1991): First record for Venezuela, new altitudinal record, and distribution map. Check List 6, 460-462.

5 Rada, M., Rueda-Almonacid, J. V., Velásquez-Álvarez, Á. A. \& Sánchez-Pacheco, S. J. 2007 Descripción de las larvas de dos centrolénidos (Anura: Centrolenidae) del noroccidente de la Cordillera Oriental, Colombia. Pap. Avulsos Zool. 47, 259-272.

6 Cisneros-Heredia, D. F. \& Yánez-Muñoz, M. H. 2007 Amphibia, Anura, Centrolenidae, Centrolene balionotum, Centrolene geckoideum, and Cochranella cariticommata: Distribution extension, new provincial records, Ecuador. Check List 3, 39-42.

7 Rodríguez Mercado, D. A. 2012 Rulyrana erminea (Torres-Gastello, Suárez-Segovia and Cisneros-Heredia, 2007) (Anura: Centrolenidae): Distribution and range extension, Peru. Check List 8 , 894-895.

8 Ospina-Sarria, J. J., Bolívar-G, W. \& Mendez-Narvaez, J. 2010 Amphibia, Anura, Centrolenidae, Espadarana callistomma (Guayasamin and Trueb, 2007): First country records from Colombia. Check List 6, 244-245.
9 Osorio-Dominguez, D. \& Quintero-Angel, A. 2012 Espadarana prosoblepon Boettger, 1892 (Amphibia: Anura): Distribution extension on the western slopes of the Cordillera Central, Colombia. Check List 8, 898-899.

10 Noronha, C. De, Rodrigues, D. D. J. \& Barros, A. B. 2012 New record and distribution map of Hyalinobatrachium cappellei (van Lidth de Jeude 1904) (Anura: Centrolenidae). Herpetol. Notes 5, 467-468.

11 Simões, P. I., Kaefer, I. L., RodriguesGomes, F. B. \& Pimentel-Lima, A. 2012 Distribution extension of Hyalinobatrachium cappellei (van Lidth de Jeude, 1904) (Anura: Centrolenidae) across Central Amazonia. Check List 8, 636-637.

12 Rodrigues, D. D. J., Lima, M. D. M., Morais, D. H. \& Kawashita-Ribeiro, R. A. 2010 Amphibia, Anura, Centrolenidae, Hyalinobatrachium crurifasciatum Myers and Donnelly, 1997: First record from Brazil and geographic distribution map. Check List 6, 392-394.

13 Castroviejo-Fisher, S., Vilà, C., Ayarzagüena, J., Blanc, M. \& Ernst, R. 2011 Species diversity of Hyalinobatrachium glassfrogs (Amphibia: Centrolenidae) from the Guiana Shield, with the description of two new especies. Zootaxa $1-55$.

14 Cisneros-Heredia, D. F., Strüssmann, C., Ávila, R. W. \& Kawashita-Ribeiro, R. A. 2010 Amphibia, Anura, Centrolenidae, Hyalinobatrachium carlesvilai Castroviejo-Fisher, Padial, Chaparro, Aguayo and de la Riva, 2009: First country record, Brazil. Check List 6, 225-226.

15 Acosta-Galvis, A. R. 2012 First record of Hyalinobatrachium fleischmanni (Boettger, 1893) (Anura: Centrolenidae) from the Caribbean region of Colombia. Check List 8, 794-795.

16 Guayasamin, J. M. \& North, S. 2009 Amphibia, Centrolenidae, Hyalinobatrachium iaspidiense: Distribution extension. Check List 5, 526-529. 
17 Yánez-Muñoz, M., Pérez-Peña, P. E. \& Cisneros-Heredia, D. F. 2009 New country records of Hyalinobatrachium iaspidiense (Amphibia, Anura, Centrolenidae) from the Amazonian lowlands of Ecuador and Peru. Herpetol. Notes 2, 49-52.

18 Castroviejo-Fisher, S., Moravec, J., Aparicio, J., Guerrero-Reinhard, M. \& Calderón, G. 2011 DNA taxonomy reveals two new species records of Hyalinobatrachium (Anura: Centrolenidae) for Bolivia. Zootaxa 2798, 64-68.

19 Castroviejo-Fisher, S., Señaris, J. C., Ayarzagüena, J. \& Vilà, C. 2008 Resurrection of Hyalinobatrachium orocostale and Notes on the Hyalinobatrachium orientale Species Complex (Anura: Centrolenidae). Herpetologica 64, 472-484. (doi:10.1655/07-049R2.1)

20 Castroviejo-Fisher, S., Padial, J. M., Chaparro, J. C., Aguayo, R. \& De la Riva, I. 2009 A new species of Hyalinobatrachium (Anura: Centrolenidae) from the Amazonian slopes of the central Andes, with comments on the diversity of the genus in the area. Zootaxa 2143, 24-44.

21 Cisneros-Heredia, D. F. \& McDiarmid, R. W. 2005 Amphibia, Centrolenidae, Centrolene peristictum, Centrolene prosoblepon, Cochranella cochranae, Cochranella midas, Cochranella resplendens, Cochranella spinosa, Hyalinobatrachium munozorum: Range extensions and new provincial records. Check List 1, 18-22.

22 Guayasamin, J. M., Lehr, E., Rodríguez, D. \& Aguilar, C. 2006 A new species of glass frog (Centrolenidae: Cochranella ocellata group) from Central Peru. Herpetologica 62, 163-172. (doi:10.1655/05-21.1)

23 Cisneros-Heredia, D. F. 2009 Amphibia, Anura, Centrolenidae, Chimerella mariaelenae (Cisneros-Heredia and McDiarmid, 2006), Rulyrana flavopunctata (Lynch and Duellman, 1973), Teratohyla pulverata (Peters, 1873), and Teratohyla spinosa (Taylor, 1949): Historical records, distribution. Check List 5, 912-916.
24 Ortega-Andrade, H. M., Bermingham, J., Aulestia, C. \& Paucar, C. 2010 Herpetofauna of the Bilsa Biological Station, province of Esmeraldas, Ecuador. Check List 6, 13-25.

25 Cisneros-Heredia, D. F., Yánez-Muñoz, M. H. \& Ortega-Andrade, M. 2009 Description of a new species of Teratohyla Taylor (Amphibia: Athesphatanura: Centrolenidae) from northwestern Ecuador. Zootaxa 2227, 53-62.

26 Oliveira, U. S., Meneghelli, D. \& Messias, M. 2012 Amphibia, Anura, Centrolenidae, Cochranella adenocheira Harvey and Noonan, 2005: Distribution and geographic extension in the state of Rondônia, Brazil. Check List 8, 145-146.

27 Toledo, L. F., Araújo, O. G., Ávila, R. W., Kawashita-Ribeiro, R. A., Morais, D. H. \& CisnerosHeredia, D. F. 2009 Amphibia, Anura, Centrolenidae, Cochranella adenocheira: distribution and range extension, Brazil. Check List 5, 380-382.

28 Kok, P. J. R. \& Castroviejo-Fisher, S. 2008 Glassfrogs (Anura: Centrolenidae) of Kaieteur National Park, Guyana, with notes on the distribution and taxonomy of some species of the family in the Guiana Shield. Zootaxa 1680, 25-53.

29 Castroviejo-Fisher, S., Guayasamin, J. M. \& Kok, P. J. R. 2009 Species status of Centrolene lema Duellman and Señaris, 2003 (Amphibia: Centrolenidae) revealed for Integrative Taxonomy. Zootaxa 1980, 16-28.

30 Savaris, M., Lampert, S., Lucas, E. M., Peres, A. V. da R., Orsato, J., Rezende, É. L. \& Argerich, G. R. 2011 Amphibia, Anura, Centrolenidae, Vitreorana uranoscopa (Müller, 1924): New record for the northeastern region of the state of Rio Grande do Sul, Brazil. Check List 7, 841-842.

31 São-Pedro, V. de A. \& Feio, R. N. 2011 Anuran species composition from Serra do Ouro Branco, southernmost Espinhaço Mountain Range, state of Minas. Check List 7, 671-680. 
32 Machado, I. F., Bairos-Moreira, L. F., Silva, R. B., Becker, R. G. \& Oliveira-Mesquita, A. S. 2010 Amphibia, Anura, Centrolenidae, Vitreorana uranoscopa (Müller, 1924): Distribution extension in the state of Rio Grande do Sul, Brazil. Check List 6, 410-411.

33 Almeida, A. P., Gasparini, J. L. \& VieiraPeloso, P. L. 2011 Frogs of the state of Espírito Santo, southeastern Brazil - The need for looking at the "coldspots." Check List 7, 542-560.

34 Rivero, J. A. 1985 Nuevos Centrolenidos de Colombia y Venezuela. Brenesia 23, 335-373.

35 Señaris, J. C. \& Ayarzagüena, J. 2005 Revisión taxonómica de la familia Centrolenidae (Amphibia; Anura) de Venezuela. Publicaciones del Com. Español del Programa Hombre y Biosf. IberoMaB la UNESCO. 7, 1-337.

36 IUCN 2012 The IUCN Red List of Threatened Species. Version 2012.2 , <http://www.iucnredlist.org>.

37 Ayarzagüena, J. \& Señaris, J. C. 1996 Dos nuevas especies de Cochranella (Anura; Centrolenidae) para Venezuela. Publicación Asoc. Amigos Doñana 8, 1-16.

38 Guayasamin, J. M., Bustamante, M. R., Almeida-Reinoso, D. \& Funk, W. C. 2006 Glass frogs (Centrolenidae) of Yanayacu Biological Station, Ecuador, with the description of a new species and comments on centrolenid systematics. Zool. J. Linn. Soc. 147, 489-513. (doi:10.1111/j.10963642.2006.00223.x)

39 Ruiz-Carranza, P. M. \& Lynch, J. D. 1991 Ranas Centrolenidae de Colombia II. Nuevas especies de Centrolene de la Cordillera Oriental y Sierra Nevada de Santa Marta. Lozania (Acta Zool. Colomb. 58, 1-27.

40 Rivero, J. A. 1968 Los Centrolenidos de Venezuela (Amphibia, Salientia). Mem. la Soc. Ciencias Nat. La Salle 28, 301-334.
41 Ruiz-Carranza, P. M. \& Lynch, J. D. 1997

Ranas Centrolenidae de Colombia $X$. Los centrolénidos de un perfil del flanco oriental de la Cordillera Central en el Departamento de Caldas. Rev. la Acad. Colomb. Ciencias Exactas, Físicas y Nat. 21, 541-553.

42 Noble, G. K. 1920 Two new batrachians from Colombia. Bull. Am. Museum Nat. Hist. 42, 441-446.

43 Lynch, J. D. \& Duellman, W. E. 1973 A review of the Centrolenid frogs of Ecuador with descriptions of new species. Occas. Pap. Museum Nat. Hist. Univ. Kansas 16, 1-66.

44 Duellman, W. E. \& Schulte, R. 1993 New Species of Centrolenid Frogs from Northern Peru. Occas. Pap. Museum Nat. Hist. Univ. Kansas 155, 133.

45 Ruiz-Carranza, P. M. \& Lynch, J. D. 1995 Ranas Centrolenidae de Colombia VIII. Cuatro nuevas especies de Centrolene de la Cordillera Central. Lozania (Acta Zool. Colomb. 65, 1-16.

46 Wild, E. R. 1994 Two New Species of Centrolenid Frogs from the Amazonian Slope of the Cordillera Oriental, Ecuador. J. Herpetol. 28, 299310.

47 Duellman, W. E. \& Burrowes, P. A. 1989 New species of frogs, Centrolenella, from the Pacific versant of Ecuador and Southern Colombia. Occas. Pap. Museum Nat. Hist. Univ. Kansas 132, 1-14.

48 Hutter, C. R. \& Guayasamin, J. M. 2012 A new cryptic species of glassfrog (Centrolenidae: Nymphargus) from Reserva Las Gralarias, Ecuador. Zootaxa 3257, 1-21.

49 Boulenger, G. A. 1882 Catalogue of Batrachia Salientia s. Ecaudata. Collection of the British Museum.

50 Cisneros-Heredia, D. F. \& Morales-Mite, M. A. 2008 A new species of glassfrog from the elfin forests of the Cordillera del Cóndor, southeastern Ecuador (Anura: Centrolenidae). Herpetozoa 21, 49-56. 
Ranas Centrolenidae de Colombia III. Nuevas especies de Cochranella del Grupo granulosa. Lozania (Acta Zool. Colomb. 59, 1-19.

52 Cisneros-Heredia, D. F. 2007 A new species of glassfrog of the genus Centrolene from the foothills of Cordillera Oriental of Ecuador. Herpetozoa 20, 27-34.

53 Guayasamin, J. M., Castroviejo-Fisher, S., Trueb, L., Ayarzagüena, J., Rada, M. \& Vilà, C. 2009 Phylogenetic systematics of Glassfrogs (Amphibia: Centrolenidae) and their sister taxon Allophryne ruthveni. Zootaxa 2100, 1-97.

54 Duellman, W. E. 1981 Three new species of centrolenid frogs from the Pacific versant of Ecuados and Colombia. Occas. Pap. Museum Nat. Hist. Univ. Kansas 88, 1-9.

55 Cadle, J. E. \& McDiarmid, R. W. 1990 Two New Species of Centrolenella ( Anura , Centrolenidae ) from Northwestern Peru. Proc. Biol. Soc. Washingt. 103, 746-768.

56 Duellman, W. E. 1980 The identity of Centrolenella grandisonae Cochran and Goin (Anura: Centrolenidae). Trans. Kansas Acad. Sci. 83, 26-32.

57 Cisneros-Heredia, D. F. \& McDiarmid, R. W. 2007 Revision of the character of Centrolenidae (Amphibia: Anura: Athesphatanura), with comments on its taxonomy and the description of new taxa of glassfrogs. Zootaxa 1572, 1-82.

58 Flores, G. 1985 A New Centrolenella (Anura) from Ecuador, with comments on nuptial pads and prepollical spines in Centrolenella. J. Herpetol. 19, 313-320.

59 Cisneros-Heredia, D. F. \& McDiarmid, R. W. 2006 Review of the taxonomy and conservation status of the Ecuadorian Glassfrog: Cedntrolenella puyoensis Flores \& McDiarmid (Amphibia: Anura: Centrolenidae). Zootaxa 1361, 21 - 31.
Cisneros-Heredia, D. F. 2007 Cochranella erminea, a new species of Centrolenidae (Amphibia: Anura: Athesphatanura) from Amazonian Peru. J. Natl. Museum (Prague), Nat. Hist. Ser. 176, 1-12.

61 Kubicki, B. 2007 Ranas de vidrio de Costa Rica / Glass frogs of Costa Rica. Heredia, Costa Rica: Instituto Nacional de Biodiversidad (INBio).

62 Savage, J. M. 2002 The Amphibians and Reptiles of Costa Rica. A Herpetofaun between two Continents, between two seas. Chicago: The University of Chicago Press.

63 Savage, J. M. \& Starrett, P. H. 1967 A new fringe-limbed tree frog (Family Centrolenidae) from lower Central America. Copeia 1967, 604-609.

64 Guayasamin, J. M. \& Bonaccorso, E. 2004 A new species of glass frog (Centrolenidae: Cochranella) from the lowlands of northwestern Ecuador, with comments on the Cochranella granulosa group. Herpetologica 60, 485-494.

65 Ruiz-Carranza, P. M. \& Lynch, J. D. 1996 Ranas Centrolenidae de Colombia IX. Dos nuevas especies del suroeste de Colombia. Lozania (Acta Zool. Colomb. 68, 1-11.

66 Harvey, M. B. 1996 A new species of glass frog (Anura: Centrolenidae: Cochranella) from Bolivia, and the taxonomic status of Cochranella flavidigitata. Herpetologica 52, 427-435.

67 Lötters, S. \& Köhler, J. 2000 Cochranella nola (Anura: Centrolenidae): natural history notes, distribution, and advertisement call. Herpetol. Nat. Hist. 7, 79-81.

68 Guayasamin, J. M. \& Trueb, L. 2007 A new species of Glassfrog (Anura: Centrolenidae) from the lowlands of northwestern Ecuador, with comments on centrolenid osteology. Zootaxa 1447, 27-45.

69 Boettger, O. 1893 Ein neuer Laubfrosch aus Costa Rica. Bergischen Naturfor orschenden Gesellschaft / Frankfurt 1892-1893, 251-252. 
70 Bustamante, M. R., Cisneros-Heredia, D. F., Yánez-Muñoz, M. H., Ortega-Andrade, H. M. \& Guayasamin, J. M. 2007 Amphibia, Centrolenidae, Cochranella pulverata, Hyalinobatrachium aureoguttatum: Distribution extension, Ecuador. Check List 3, 271-276.

71 Cannatella, D. C. 1980 Two new species of Centrolenella from Bolivia (Anura: Centrolenidae). Proc. Biol. Soc. Washingt. 93, 714-724.

72 Myers, C. W. \& Donnelly, M. A. 2001 Herpetofauna of the Yutajé-Corocoro Massif, Venezuela: Second report from the Robert G. Goelet American Museum-Terramar Expedition to the northwestern tepuis. Bull. Am. Museum Nat. Hist. 261, 1-85.

73 Noonan, B. P. \& Bonett, R. M. 2003 A New Species of Hyalinobatrachium (Anura: Centrolenidae) from the Highlands of Guyana. J. Herpetol. 37, 91-97.

74 Myers, C. W. \& Donnelly, M. A. 1997 A tepui herpetofauna on a granitic moun- tain (Tamacuri) in the borderland between Venezuela and Brazil: report from the Phipps Tapirapecó expedition. Am. Museum Novit. 3213, 1-71.

75 Taylor, E. H. 1958 Notes on Costa Rican Centrolenidae with descriptions of new forms. Univ. Kansas Sci. Bull. 39.

76 Taylor, E. H. 1949 Costa Rican frogs of the genera Centrolene and Centrolenella. Univ. Kansas Sci. Bull. 33, 257-270.

77 Ayarzagüena, J. 1992 Los Centrolenidos de la Guayana Venezolana. Publicación Asoc. Amigos Doñana 1, 1-48.

78 Lescure, J. \& Marty, C. 2000 Atlas des Amphibiens de Guyane. Patrimoines Nat. 45, 1-338.

79 Señaris, J. C. \& Ayarzagüena, J. 2001 Una nueva especie de rana de cristal del género Hyalinobatrachium (Anura; Centrolenidae) del delta del río Orinoco. Rev. Biol. Trop. 49, 1007-1017.
80 Ruiz-Carranza, P. M. \& Lynch, J. D. 1998 Ranas Centrolenidae de Colombia XI. Nuevas especies de ranas cristal del genero Hyalinobatrachium. Rev. la Acad. Colomb. Ciencias Exactas, Físicas y Nat. 22, 571-586.

81 Taylor, E. H. 1952 The frogs and toads of Costa Rica. Univ. Kansas Sci. Bull. 35, 577-942.

82 Starrett, P. H. \& Savage, J. M. 1973 The systematic status and distribution of Costa Rica glass-frogs genus Centrolenella (family Centrolenidae), with the description of a new species. Bull. South. Calif. Acad. Sci. 72, 57-78.

83 Castroviejo-Fisher, S., Ayarzagüena, J. \& Vilà, C. 2007 A new species of Hyalinobatrachium (Centrolenidae: Anura) from Serranía de Perijá, Venezuela. Zootaxa 1441, 51-62.

84 Goin, C. J. 1968 A new centrolenid frog from Guyana. Q. J. Florida Acad. Sci. 30, 115-118.

85 Dunn, E. R. 1931 New frogs from Panama and Costa Rica. Occas. Pap. Bost. Soc. Nat. Hist. 5, 385-401.

86 Goin, C. J. 1961 Three new centrolenid frogs from Ecuador. Zool. Anz. 166, 95-104.

87 Ruiz-Carranza, P. M. \& Lynch, J. D. 1995 Ranas Centrolenidae de Colombia V. Cuatro nuevas especies de Cochranella de la Cordillera Central. Lozania (Acta Zool. Colomb. 62, 1-24.

88 Cochran, D. M. \& Goin, C. J. 1970 Frogs of Colombia. Bull. United States Natl. Museum 288, 1655.

89 Boulenger, G. A. 1918 Descriptions of new South American batrachians. Ibid 9, 427-433.

90 Duellman, W. E. 1976 Centrolenid Frogs from Peru. Occas. Pap. Museum Nat. Hist. Univ. Kansas 52, 1-11.

91 Cannatella, D. C. \& Duellman, W. E. 1982 Two new species of Centrolenella with a brief review of the genus in Peru and Bolivia. Herpetologica 38, 380-388. 
Flores, G. \& McDiarmid, R. W. 1989 Two new species of South American Centrolenella (Anura: Centrolenidae) related to C. mariae. Herpetologica , 401-411.

93 Velásquez-Álvarez, Á. A., Rada, M., Sánchez-Pacheco, S. J. \& Acosta-Galvis, A. R. 2007 A New Species of Glassfrog (Anura: Centrolenidae) from the Western Slope of the Cordillera Oriental, Colombia. South Am. J. Herpetol. 2, 191-197.

94 Ruiz-Carranza, P. M. \& Lynch, J. D. 1991 Ranas Centrolenidae de Colombia IV. Nuevas especies de Cochranella del Grupo ocellata de la Cordillera Oriental. Lozania (Acta Zool. Colomb. 60, 1-14.

95 Harvey, M. B. \& Noonan, B. P. 2005 Bolivian glass frogs (Anura: Centrolenidae) with a description of a new species from Amazonia. Proc. Biol. Soc. Washingt. 118, 428-441. (doi:10.2988/0006324X(2005)118[428:BGFACW]2.0 .CO;2)

96 Savage, J. M. 1967 A new treefrog (Centrolenidae) from Costa Rica. Copeia 1967, 325331.

97 Cisneros-Heredia, D. F. \& Meza-Ramos, P. 2007 An enigmatic new species of glassfrog (Amphibia: Anura: Centrolenidae) from the Amazonian Andean slopes of Ecuador. Zootaxa 41, 33-41.

98 Guayasamin, J. M., Castroviejo-Fisher, S., Ayarzagüena, J., Trueb, L. \& Vilà, C. 2008 Phylogenetic relationships of glassfrogs (Centrolenidae) based on mitochondrial and nuclear genes. Mol. Phylogenet. Evol. 48, 574-95. (doi:10.1016/j.ympev.2008.04.012)

99 Peters, W. C. H. 1873 Über eine neue Schildkrötenart, Cinosternon Effeldtii und einige andere neue oder weniger bekannte Amphibien. Monatsberichte der Königlich Preuss. Akad. der Wissenschaften zu Berlin 1873, 603-618.

100 Goin, C. J. 1963 A new centrolenid frog from Venezuela. Acta Biol. Venez. 3, 283-286.
101 Lutz, A. 1925 Batraciens du Bresil. Comptes Rendus Hebd. des Seances Mem. la Soc. Biol. Paris 93, 137-139.

102 Heyer, W. 1985 Taxonomic and Natural History Notes on Frogs of the Genus Centrolenella (Amphibia: Centrolenidae) from Southeastern Brasil and adjacent Argentina. Pap. Avulsos Zool. 36, 121.

103 Salles, R. O. L., Weber, L. N. \& Silva-Soares, T. 2009 Amphibia, Anura, Parque Natural Municipal da Taquara, municipality of Duque de Caxias, state of Rio de Janeiro, southeastern Brazil. Check List 5, 840-854.

104 Lescure, J. 1975 Contribution a l'etude des Amphibiens de Guyane Francaise V. Les Centrolenidae. Société Zool. Fr. , 385-394.

105 Müller, L. 1924 Neue LaubfrOsche aus dem Staate Santa Catherina, S.O. Brazilien. Zoolgischen Anzeiger 59, 233-238. 